\title{
A Versatile Stereoselective Synthesis of endo,exo-furofuranones: Application to the Enantioselective Synthesis of Furofuran Lignans
}

Nigel A. Swain, Richard C. D. Brown* and Gordon Bruton ${ }^{\dagger}$

Department of Chemistry, The University of Southampton, Highfield, Southampton SO17 1BJ, UK

${ }^{\dagger}$ GlaxoSmithKline Pharmaceuticals, New Frontiers Science Park, Harlow, Essex CM19 5AW, UK

Corresponding author email: $\underline{\text { rcb1@,soton.ac.uk }}$

Supporting Information

\begin{tabular}{|c|c|c|c|}
\hline S1 & Index & $\mathrm{S} 25$ & ${ }^{1} \mathrm{H}$ NMR for compound $\mathbf{3 7 b}$ \\
\hline S2 & ${ }^{1} \mathrm{H}$ NMR for compound $\mathbf{1}$ & S26 & ${ }^{1} \mathrm{H}$ NMR for compound $\mathbf{4 1 a}$ \\
\hline S3 & ${ }^{13} \mathrm{C}$ NMR for compound $\mathbf{1}$ & $\mathrm{S} 27$ & ${ }^{1} \mathrm{H}$ NMR for compound $\mathbf{4 1 b}$ \\
\hline S4 & ${ }^{1} \mathrm{H}$ NMR for compound 2 & $\mathrm{~S} 28$ & Experimental and data for 14 \\
\hline S5 & ${ }^{13} \mathrm{C}$ NMR for compound 2 & S29 & Data for $\mathbf{1 5}$ \\
\hline S6 & ${ }^{1} \mathrm{H}$ NMR for compound $\mathbf{3}$ & $\mathrm{S} 30$ & Experimental and data for $\mathbf{1 8}$ \\
\hline S7 & ${ }^{13} \mathrm{C}$ NMR for compound $\mathbf{3}$ & $\mathrm{S} 31$ & Experimental and data for 19 \\
\hline S8 & ${ }^{1} \mathrm{H}$ NMR for compound 4 & $\mathrm{~S} 32$ & Experimental and data for $\mathbf{2 0}$ \\
\hline S9 & ${ }^{13} \mathrm{C}$ NMR for compound 4 & $\mathrm{~S} 33$ & Experimental and data for $\mathbf{2 2}$ \\
\hline $\mathrm{S} 10$ & ${ }^{1} \mathrm{H}$ NMR for compound $\mathbf{1 1}$ & $\mathrm{S} 34$ & Experimental and data for $\mathbf{2 4}$ \\
\hline $\mathrm{S} 11$ & ${ }^{1}$ H NMR for compound 14 & $\mathrm{~S} 35$ & Experimental and data for $\mathbf{2 5}$ \\
\hline S12 & ${ }^{1} \mathrm{H}$ NMR for compound $\mathbf{1 5}$ & S36 & Experimental and data for $\mathbf{2 6}$ \\
\hline $\mathrm{S} 13$ & ${ }^{1} \mathrm{H}$ NMR for compound $\mathbf{1 6}$ & $\mathrm{S} 37$ & Experimental and data for $\mathbf{2 8}$ \\
\hline $\mathrm{S} 14$ & ${ }^{1}$ H NMR for compound $\mathbf{1 7}$ & $\mathrm{S} 38$ & Experimental and data for $\mathbf{2 9}$ \\
\hline S15 & ${ }^{1}$ H NMR for compound $\mathbf{2 0}$ & S39 & Experimental and data for $\mathbf{3 0}$ \\
\hline $\mathrm{S} 16$ & ${ }^{1}$ H NMR for compound $\mathbf{2 1}$ & $\mathrm{S} 40$ & Experimental and data for $\mathbf{3 6 a}$ \\
\hline S17 & ${ }^{1}$ H NMR for compound 22 & S41 & Experimental and data for $\mathbf{3 7 a}$ \\
\hline $\mathrm{S} 18$ & ${ }^{1}$ H NMR for compound $\mathbf{2 4}$ & S42 & Experimental and data for $\mathbf{3 8 a}$ \\
\hline S19 & ${ }^{1}$ H NMR for compound 25 & $\mathrm{~S} 43$ & Experimental and data for $\mathbf{4 0 a}$ \\
\hline S20 & ${ }^{1} \mathrm{H}$ NMR for compound $\mathbf{3 1}$ & $\mathrm{S} 44$ & Data for 41a \\
\hline $\mathrm{S} 21$ & ${ }^{1} \mathrm{H}$ NMR for compound $\mathbf{3 3}$ & $\mathrm{S} 45$ & Experimental and data for $\mathbf{4 2 a}$ \\
\hline $\mathrm{S} 22$ & ${ }^{1} \mathrm{H}$ NMR for compound 34 & $\mathrm{~S} 46$ & Experimental and data for $\mathbf{1}$ \\
\hline $\mathrm{S} 23$ & ${ }^{1} \mathrm{H}$ NMR for compound $\mathbf{3 6 a}$ & S47 & Experimental and data for $\mathbf{2}$ \\
\hline $\mathrm{S} 24$ & ${ }^{1} \mathrm{H}$ NMR for compound $\mathbf{3 7 a}$ & S48 & General Experimental \\
\hline
\end{tabular}




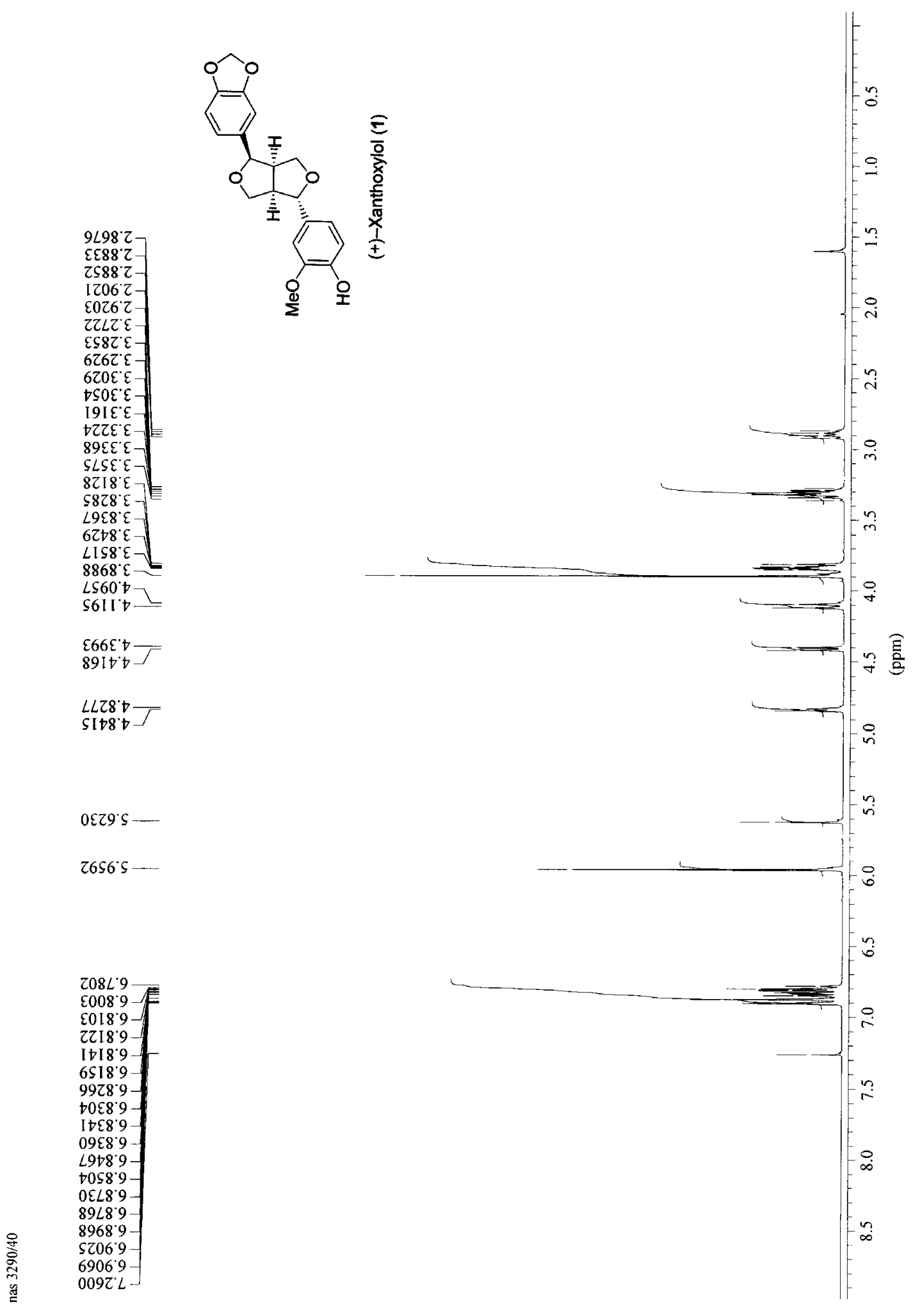



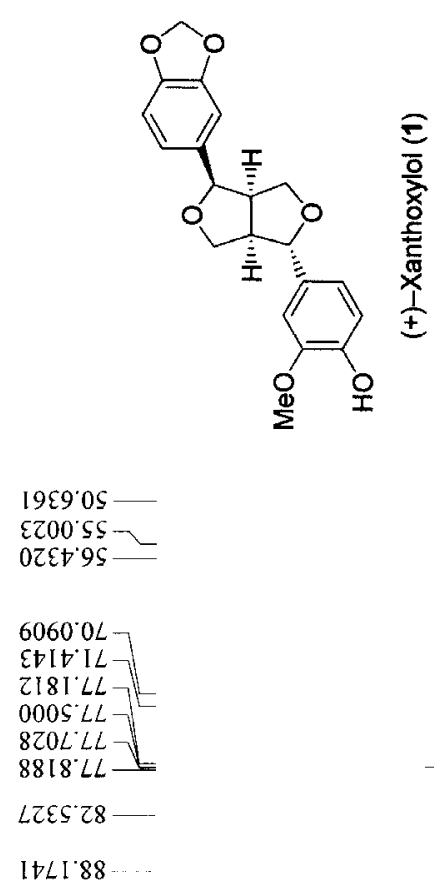

99 tt' I0I -.....

I $588^{\circ} 90 \mathrm{I}$

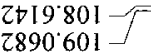

ธ8EL'tI I -

EटLI.611

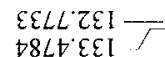

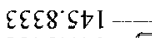

$80 t 0<\mathrm{LI}-$

$\angle t Z \angle t \mid-J$

LZZ[ $8 D I]$

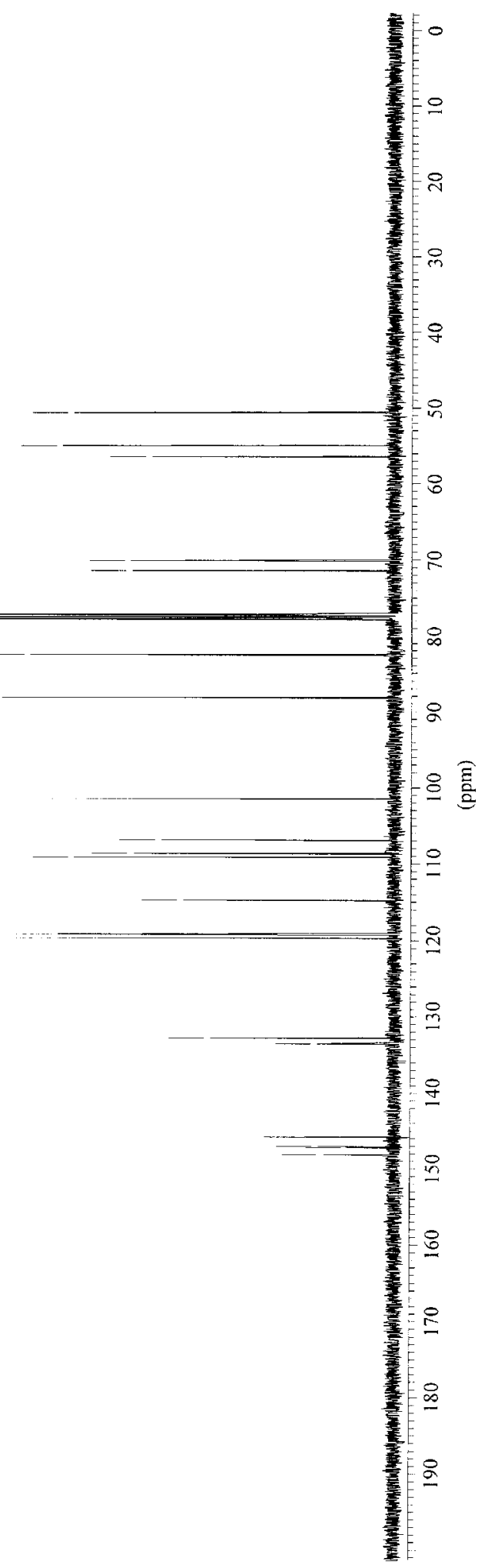




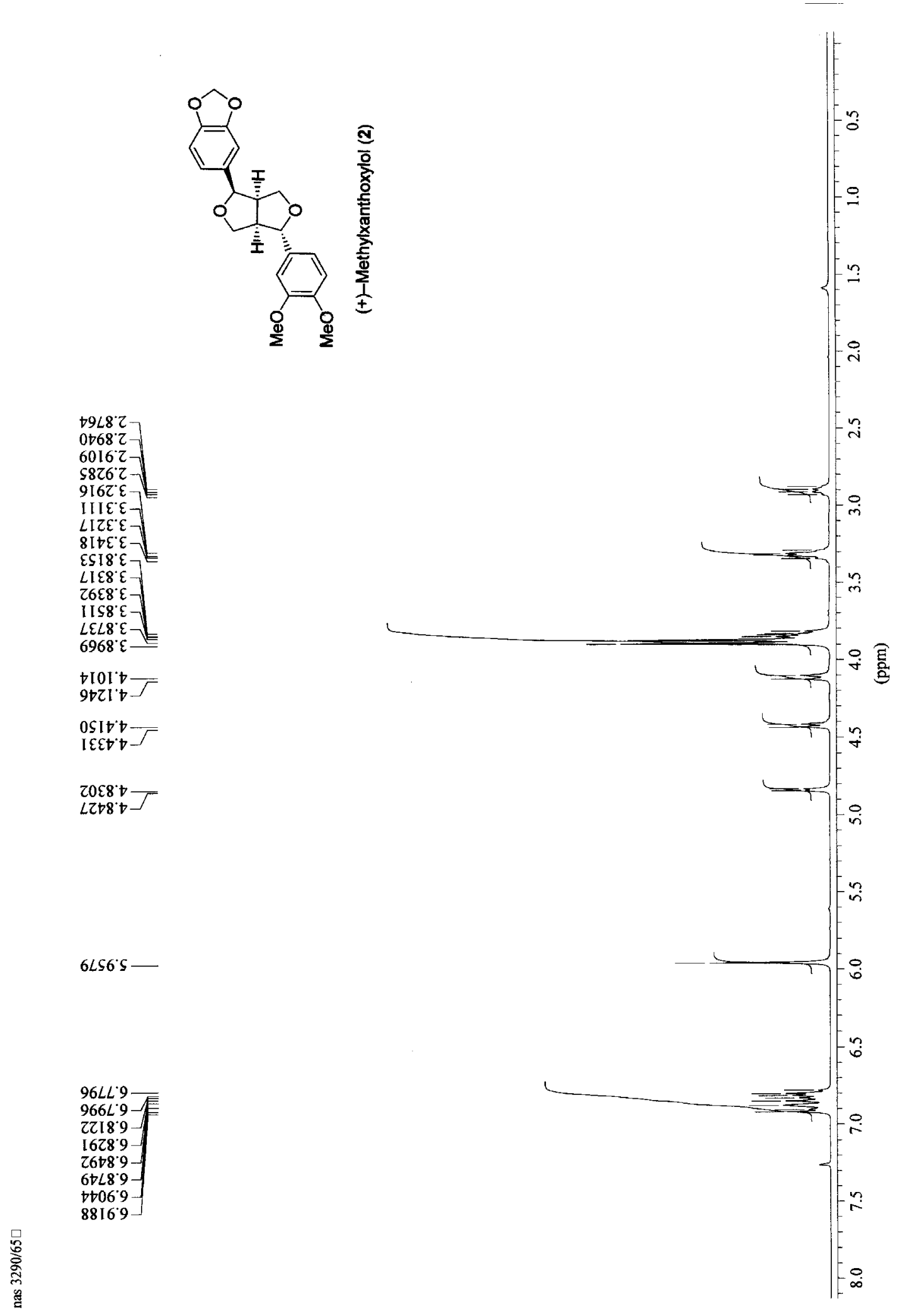



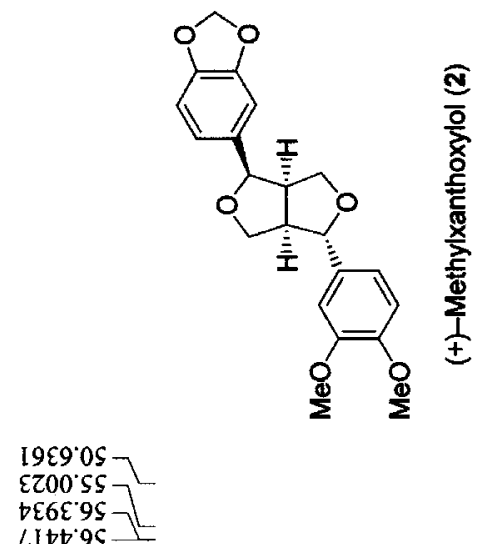

$962 I^{\circ} 0 L-$

EtIt IL $L$

ZI8I' $L L-$

$000 S^{\circ} L L$

$8818 . \mathrm{LL}$

LZ\&S'Z8 -

SLL0 88

99tt' IOI -

I $\$ 88^{\circ} 90 \mathrm{I}-$

L9L9.601

LOSS' I I I

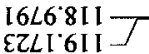

9E9LZEI -
LSZI'十EI -

†OSO $L D I-$
$\angle Z Z I \cdot 8 t I-F$
$9 \varepsilon \varepsilon Z \cdot 6 t I-$
$6 S E L 6 t=$

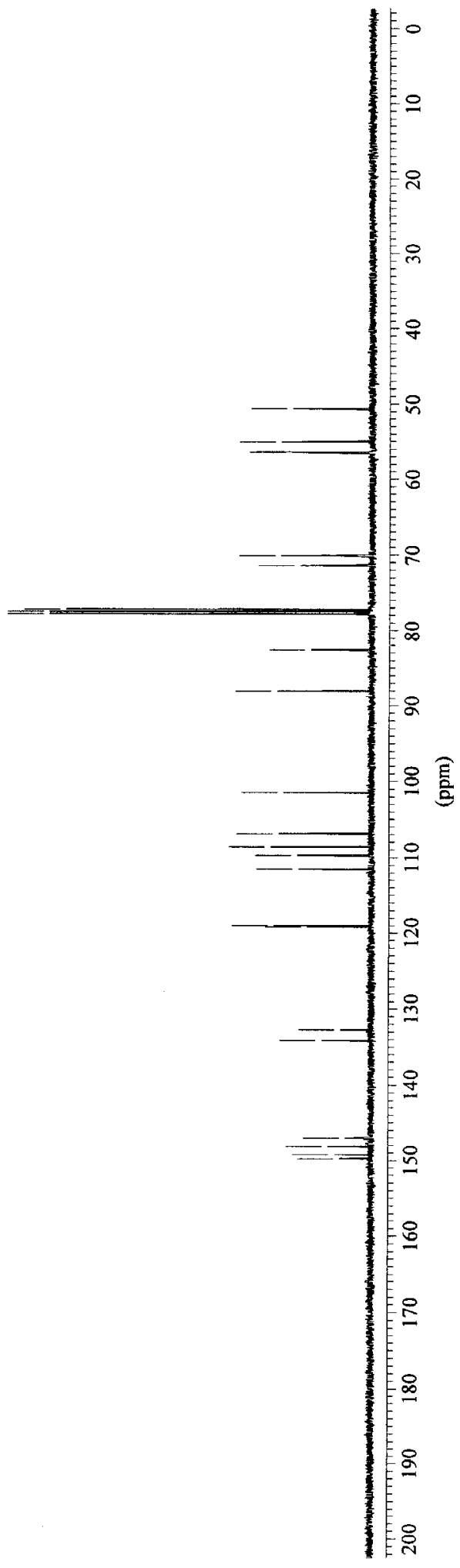




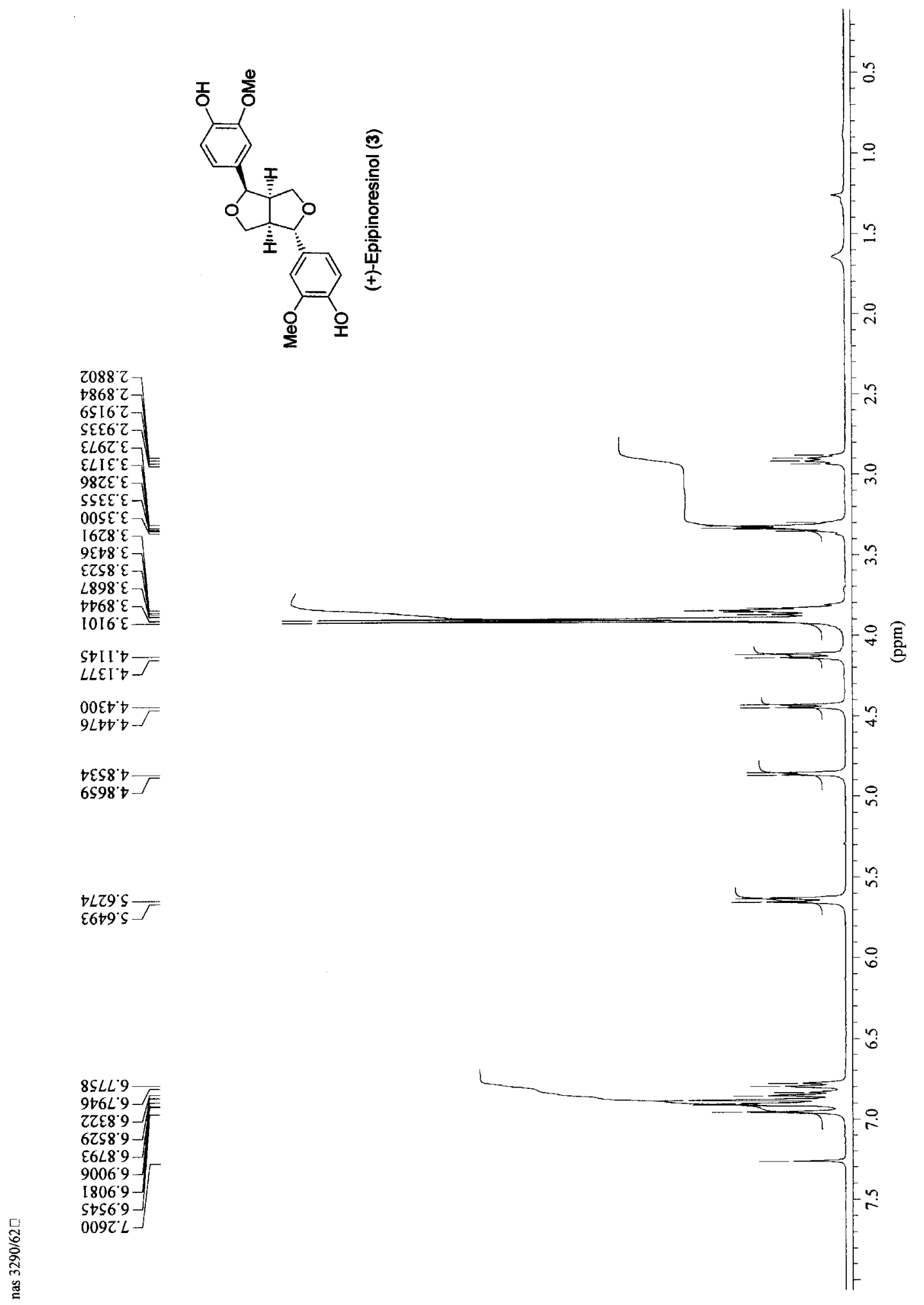




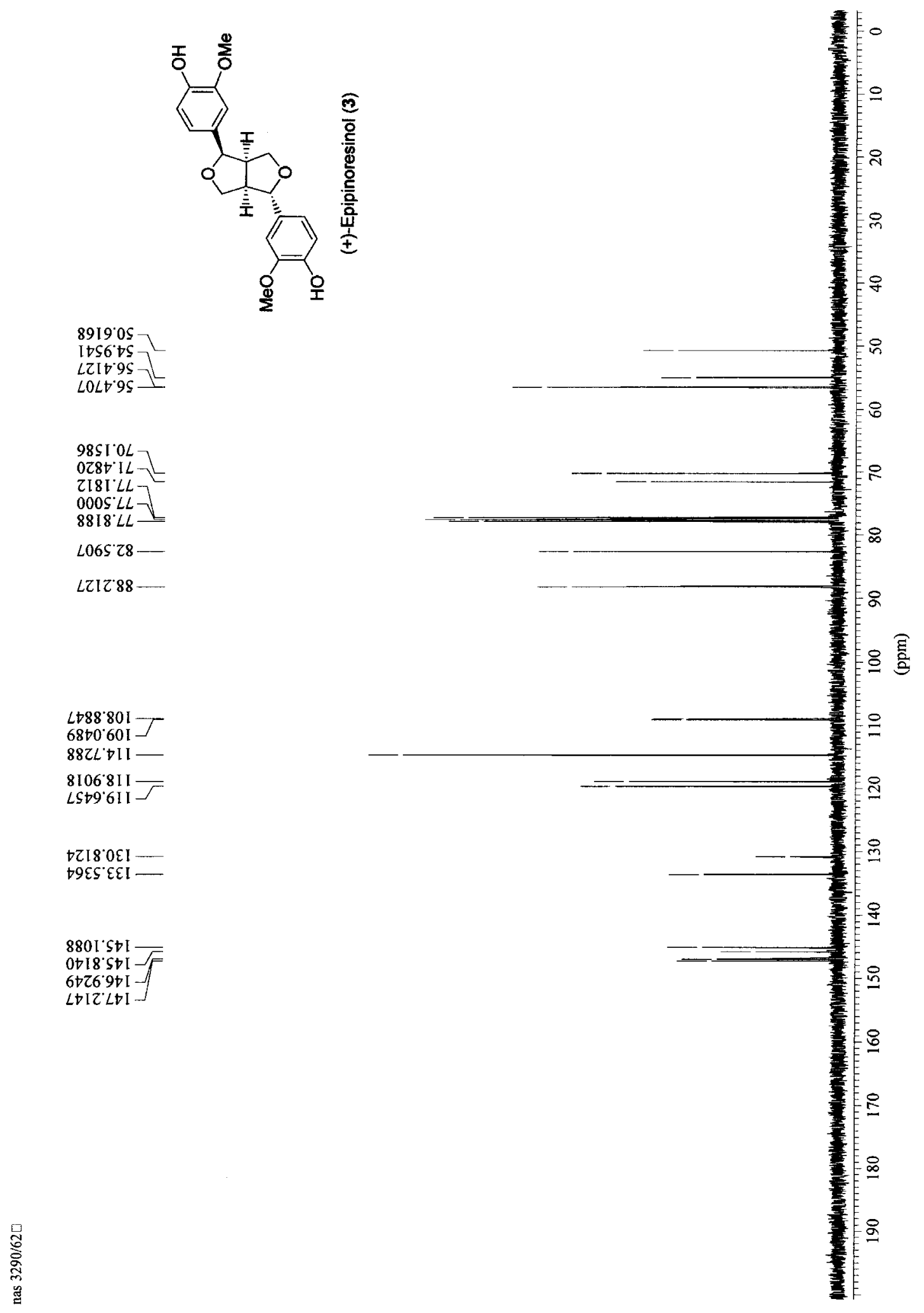




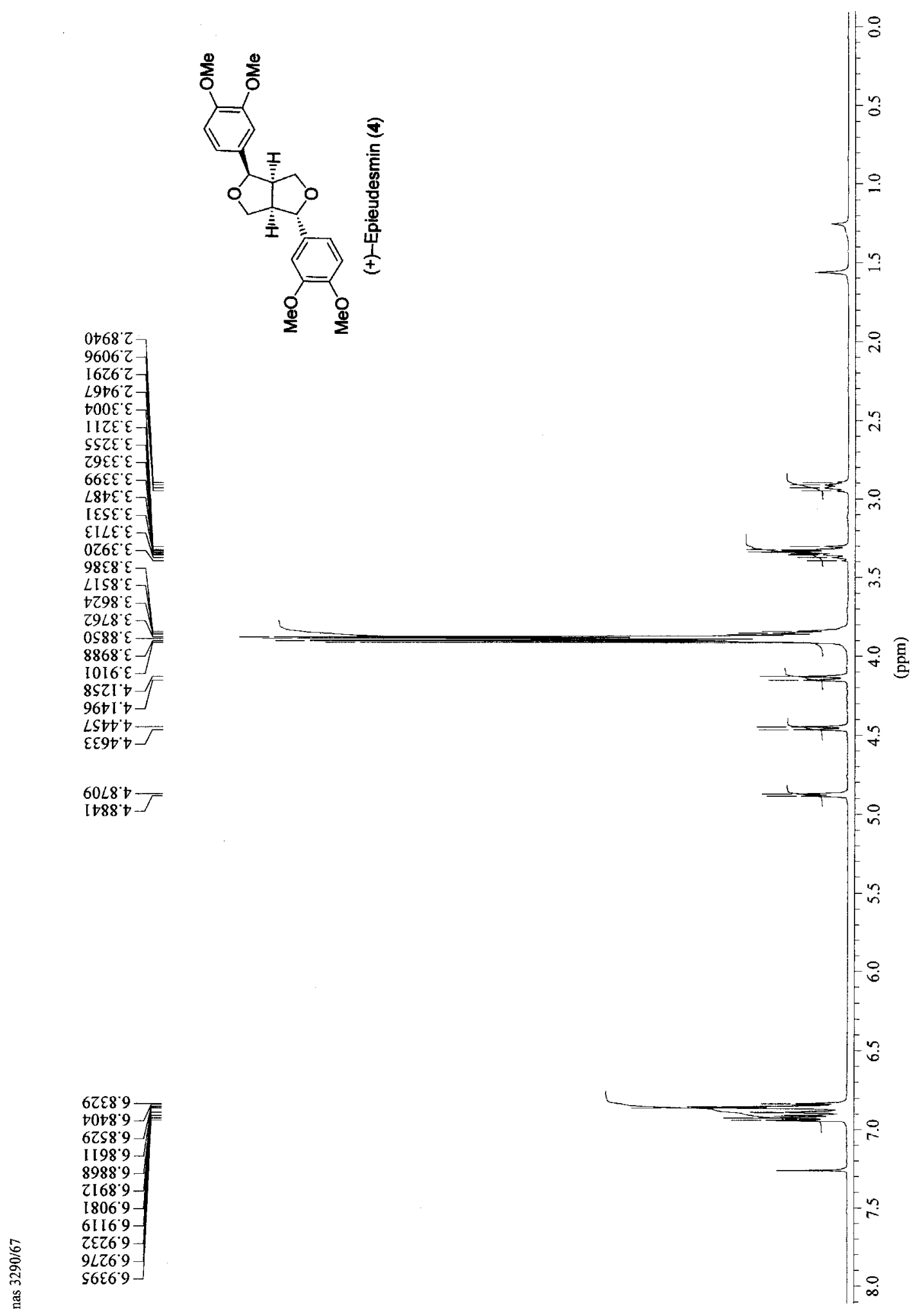




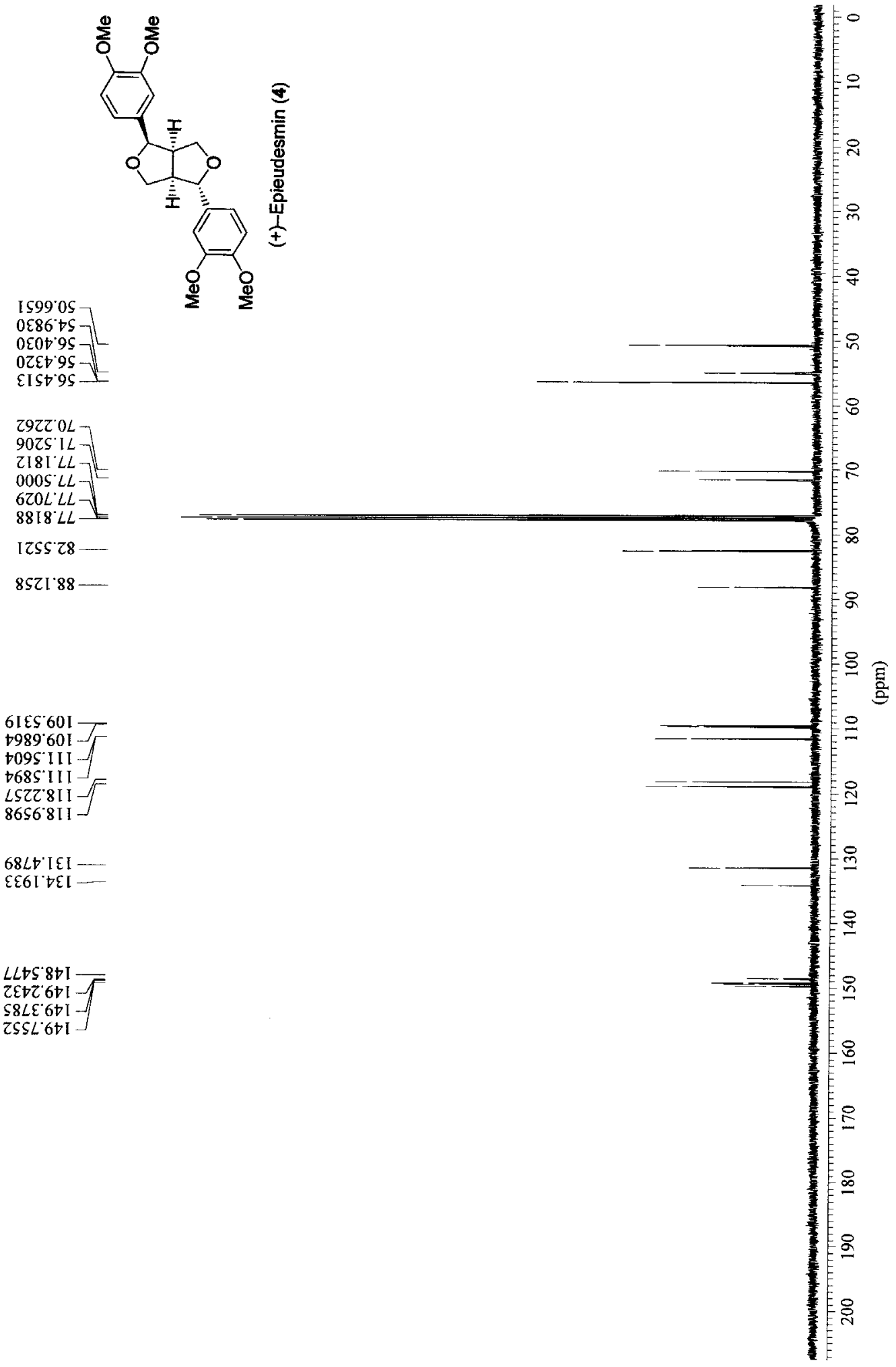




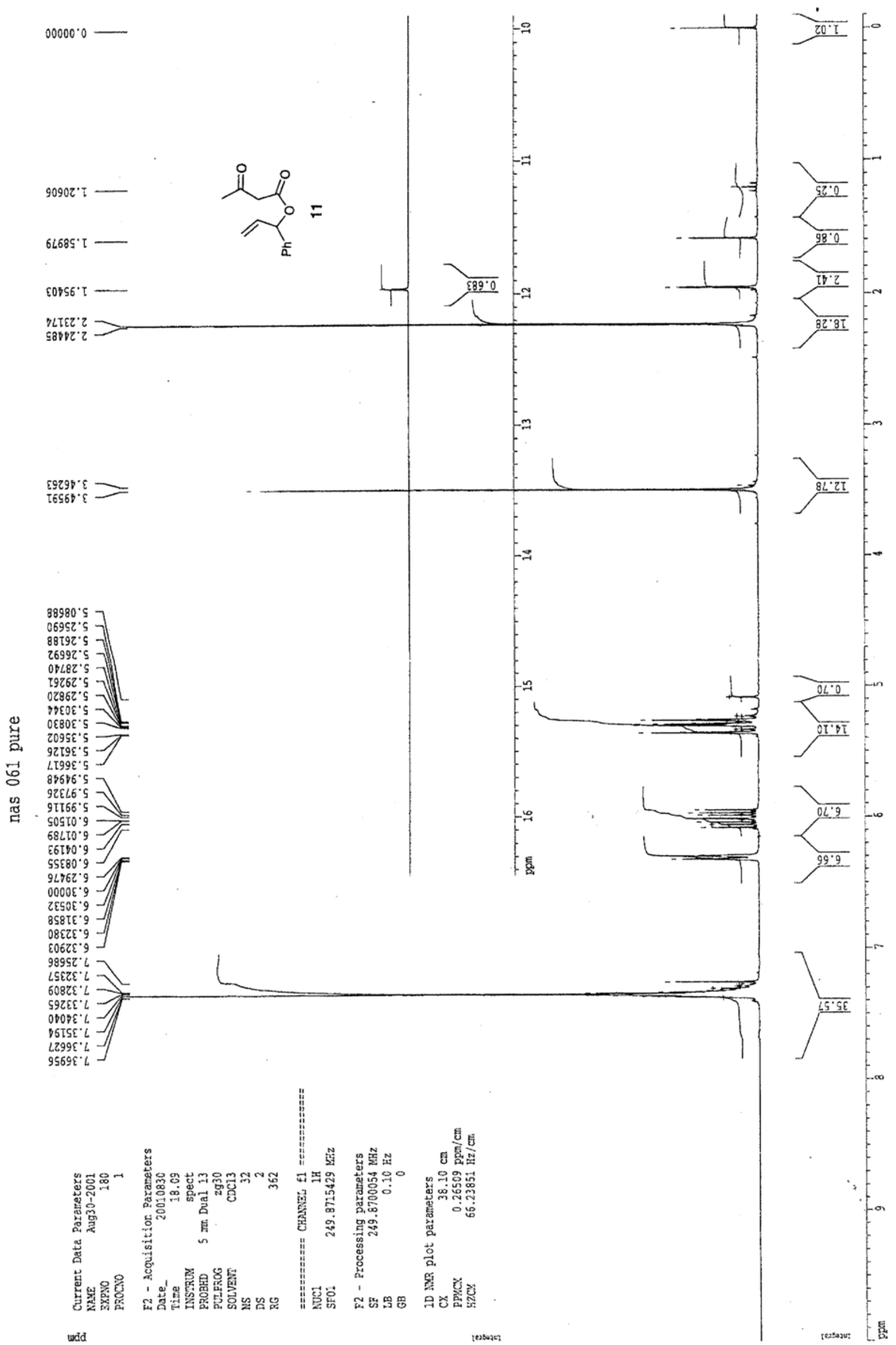




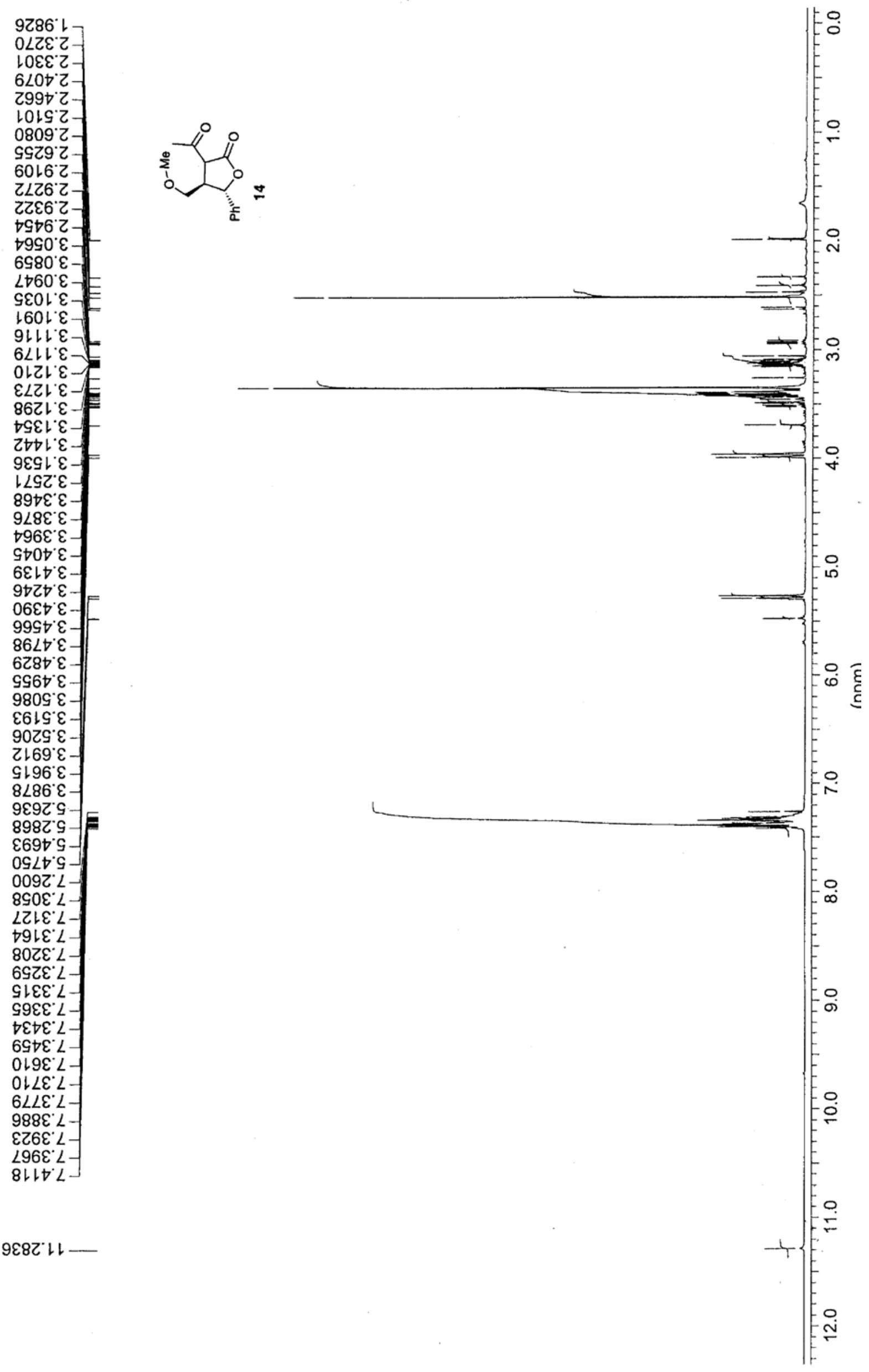




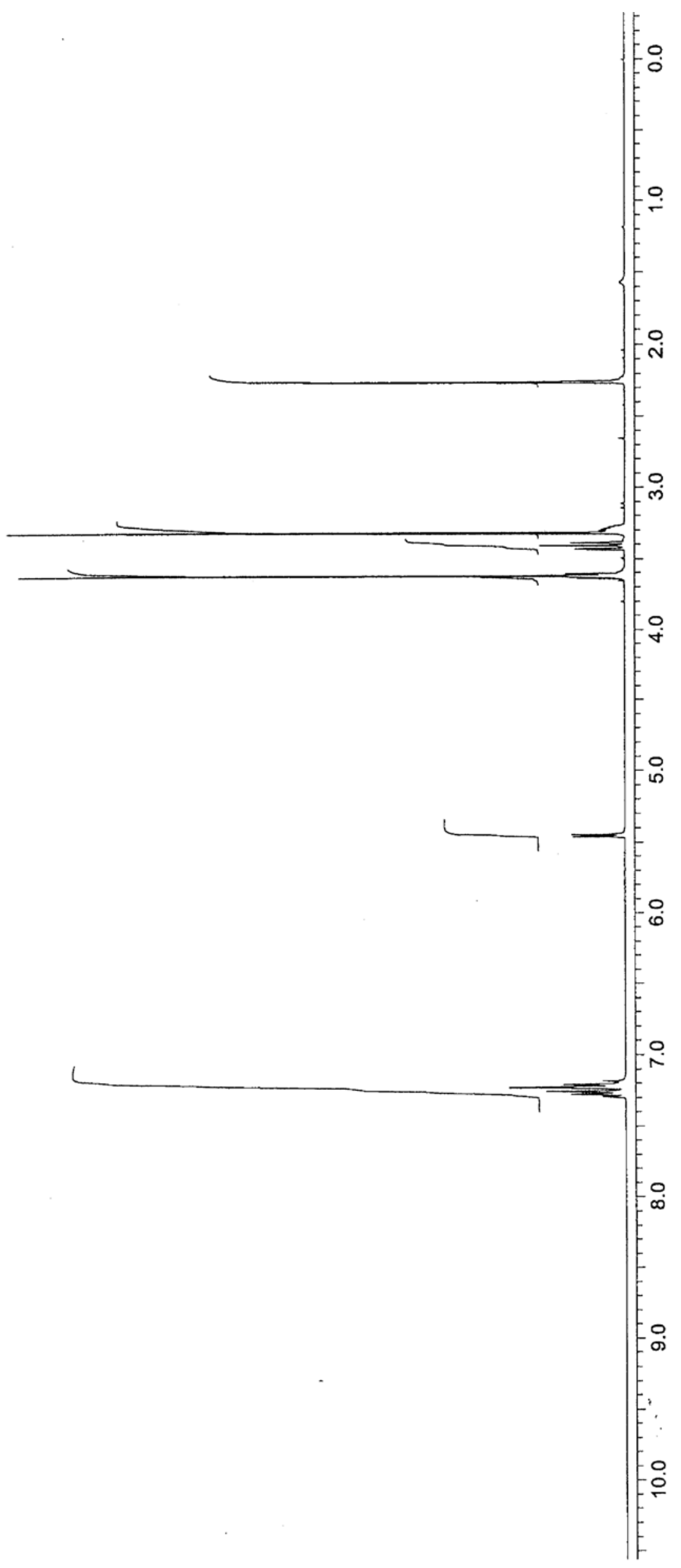


$\left.\varepsilon 6 \varepsilon 6^{\circ} \mathrm{l}\right\urcorner$

906t' $Z$

ZS80 8

$0 \triangleright 60^{\circ} \mathcal{E}$

ZZOI' $\varepsilon$

I60 I ${ }^{\circ} \varepsilon$

9 I I I $\mathcal{1}$

8 LI I ${ }^{\circ} \varepsilon$

${ }^{\circ} 0 \mathrm{ZI} \mathrm{I}^{\circ} \varepsilon$

$09 Z I^{\prime} \varepsilon$

I $6 Z I^{\circ} \mathcal{E}$

$\forall S \mathcal{\prime} I^{\prime} \varepsilon$

Z $\supset I^{\circ} \mathcal{E}$

0हSा $\varepsilon$

8L9t' $\mathcal{~}$

$09 \angle \nabla^{\circ} \varepsilon$

6265 $\varepsilon$

$\varepsilon L 6 t^{\circ} \varepsilon$

I I $0 S^{\circ} \varepsilon$

[905' $\varepsilon$

$\neg Z Z \varsigma^{\circ} \varepsilon$

8 I $\varepsilon S^{\circ} \varepsilon$

† $6 S^{\circ} \varepsilon$

$\angle L 6 S^{\circ} \varepsilon$

2019 $\varepsilon$

$\angle D 00^{\circ} \mathrm{t}$

I IEO $t$

9LOI't

EカIt゙・

OSED $\nabla$

Zह9t

LZ6"

$6 Z \nabla S^{\prime} \nabla-$

$\nabla Z L S^{\prime} \rightarrow$

I865' $\circ$.

2829.

$0 \angle S 9^{\circ} \circ$

$\varepsilon 899^{\circ} t$

Il 62 '

OSIE' $\varsigma$

LE6t'S

$8 \varepsilon 0 S^{\circ} \varsigma$

00 IS $\mathrm{S}$

9985's

$\varepsilon L I Z L$

$\angle I Z Z L$

OEZZ L

$\nabla Z E Z L$

$8 \mathrm{It} Z^{\circ} L$

9S9Z: L

$699 Z^{\circ} L$

$00 L Z^{\circ} L$

$\varepsilon 16 \tau^{\circ} L$

乙EOE L

S6IE L

$\varepsilon \varepsilon Z \varepsilon\llcorner$

$0 † \varepsilon \varepsilon^{\circ} L$

I $L E \varepsilon \varepsilon^{\circ} L$

$\mathrm{I} \angle \downarrow \varepsilon^{\circ} L$

8ZSE'L

I6S $\varepsilon^{\circ} L$

I $79 \varepsilon$ : $L$

$999 \varepsilon^{\circ} L$

L69E'L

$09 L E L$

†06E $L$

Z†6E L

IIIt L

SSIt L

$878 Z^{\circ}$ I I

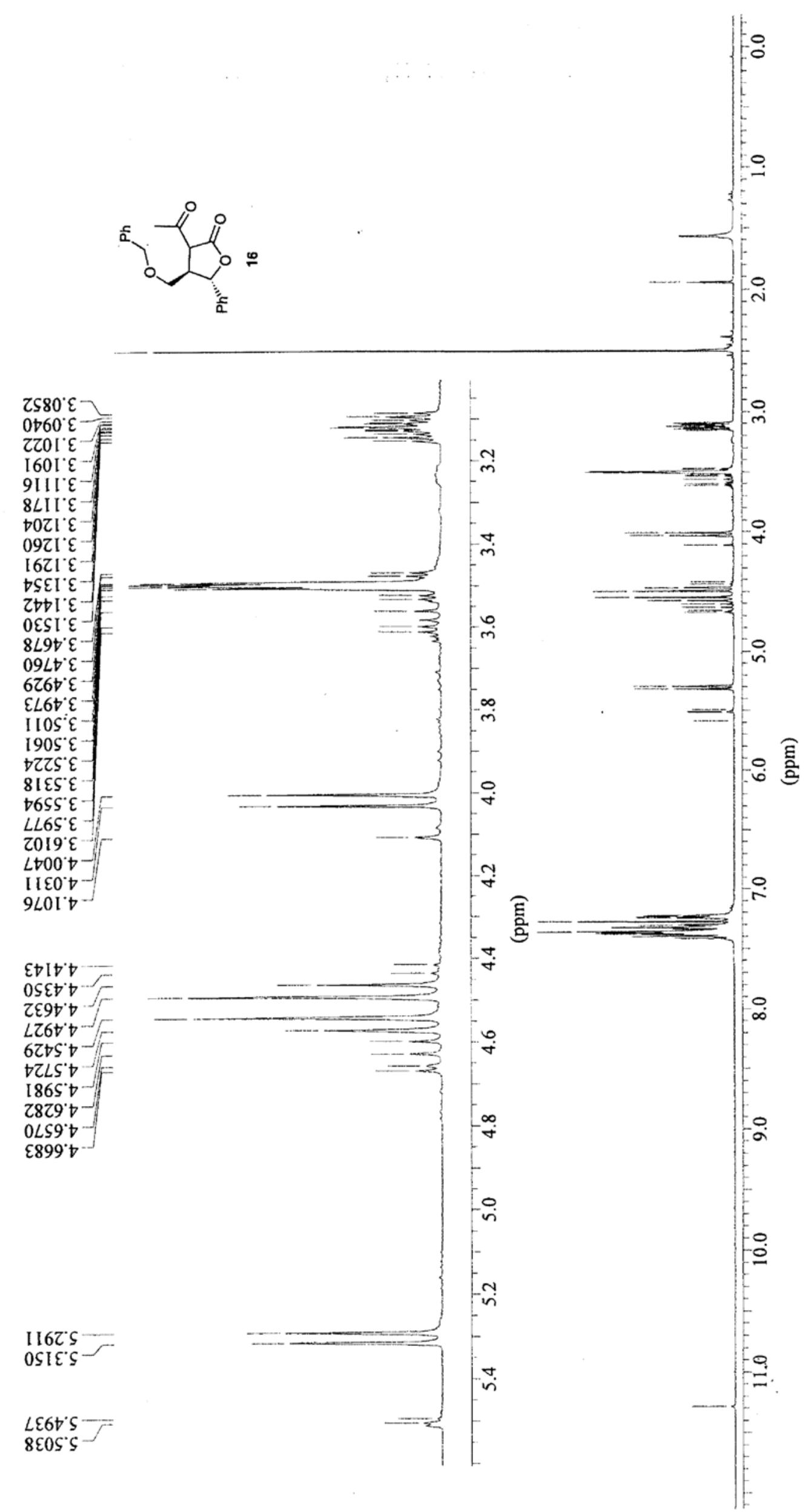




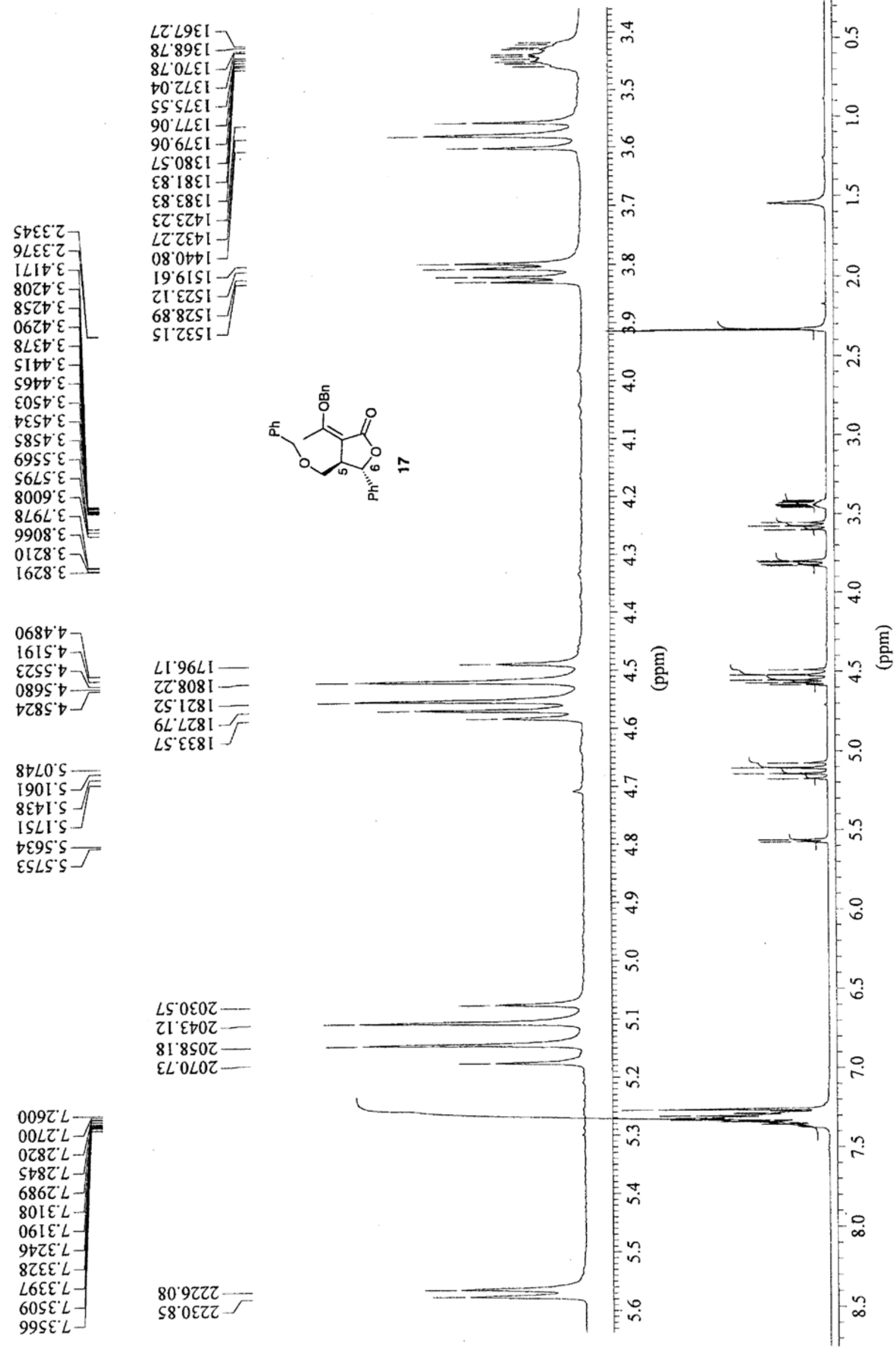


LEZ6. I -

$\angle E L D^{\circ} Z$

$\angle L 90^{\circ} \varepsilon$

ऽ $\angle L 0^{\circ} \varepsilon$

$\varepsilon S 80^{\circ} \varepsilon$

$0 \triangleright 60^{\circ} \varepsilon$

$\varepsilon 00 I^{\circ} \varepsilon$

$8 Z 0 I^{\circ} \varepsilon$

0 II I ' $\mathcal{E}$

$6 L I^{\prime} \varepsilon$

$\angle 9 Z I^{\prime} \varepsilon$

$\nabla \varsigma \varepsilon\left[{ }^{\circ} \varepsilon\right.$

$06 \varepsilon \nabla^{\circ} \varepsilon$

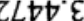

$099 \sigma^{\circ} \varepsilon$

$6 Z L \nabla^{\circ} \varepsilon-$

$\nabla S \angle \nabla^{\circ} \varepsilon$

$\varepsilon 260^{\circ} \varepsilon$

I I $0 S^{\circ} \varepsilon$

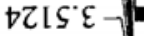

9ऽEऽ $\mathcal{E}$

9LSS $\varepsilon$

$\angle S \angle S^{\circ} \varepsilon$

$\mathcal{E} 88 \mathcal{S}^{\circ} \mathcal{E}$

$066 \varsigma^{\circ} \varepsilon-$

$\varepsilon 06 L^{\prime} \varepsilon-F$

$8 L 6 L^{\circ} \mathcal{E}$

$9908^{\circ} \mathcal{E}-$

$\varepsilon 066^{\circ} \varepsilon-$

$\varepsilon L 10^{\circ} \circ$

IEIt'†

乙Et

SI $6 \nabla^{\circ} t-$

9IZS' $\forall-$

$86 t \varsigma^{\circ} \mathrm{b}$

$8185^{\circ} \mathrm{t}$

$6 \Rightarrow L Z^{\circ} S$

[862. $\mathrm{s}$

$006 t^{\circ} \varsigma$

$0 \varsigma 60^{\circ} \varsigma$

$86 £ 8^{\circ} 9$

$\angle 978^{\circ} 9-$

$6258^{\circ} 9$

I $198^{\circ} 9$

Z†98.9-

I $998^{\circ} 9$

66L8'9

II I $Z^{\circ} L$

$\angle 9 I Z L$

I $9 Z^{\circ} L$

ऽऽE $L$

$L E t Z^{\circ} L$

$009 Z$ ' $L$

SZLZ'L

$\nabla 6 L Z L$

$\angle 06 Z^{\circ} L$

$686 Z^{\circ} L$

†I I $\varepsilon^{*} L$

LZZE L

$60 \varepsilon \varepsilon^{\circ} L$

ऽ9EE' $L$

$\angle \nabla t \varepsilon^{\circ} L$.

$\downarrow 8 \nabla \varepsilon \cdot L$.

8LSEL -

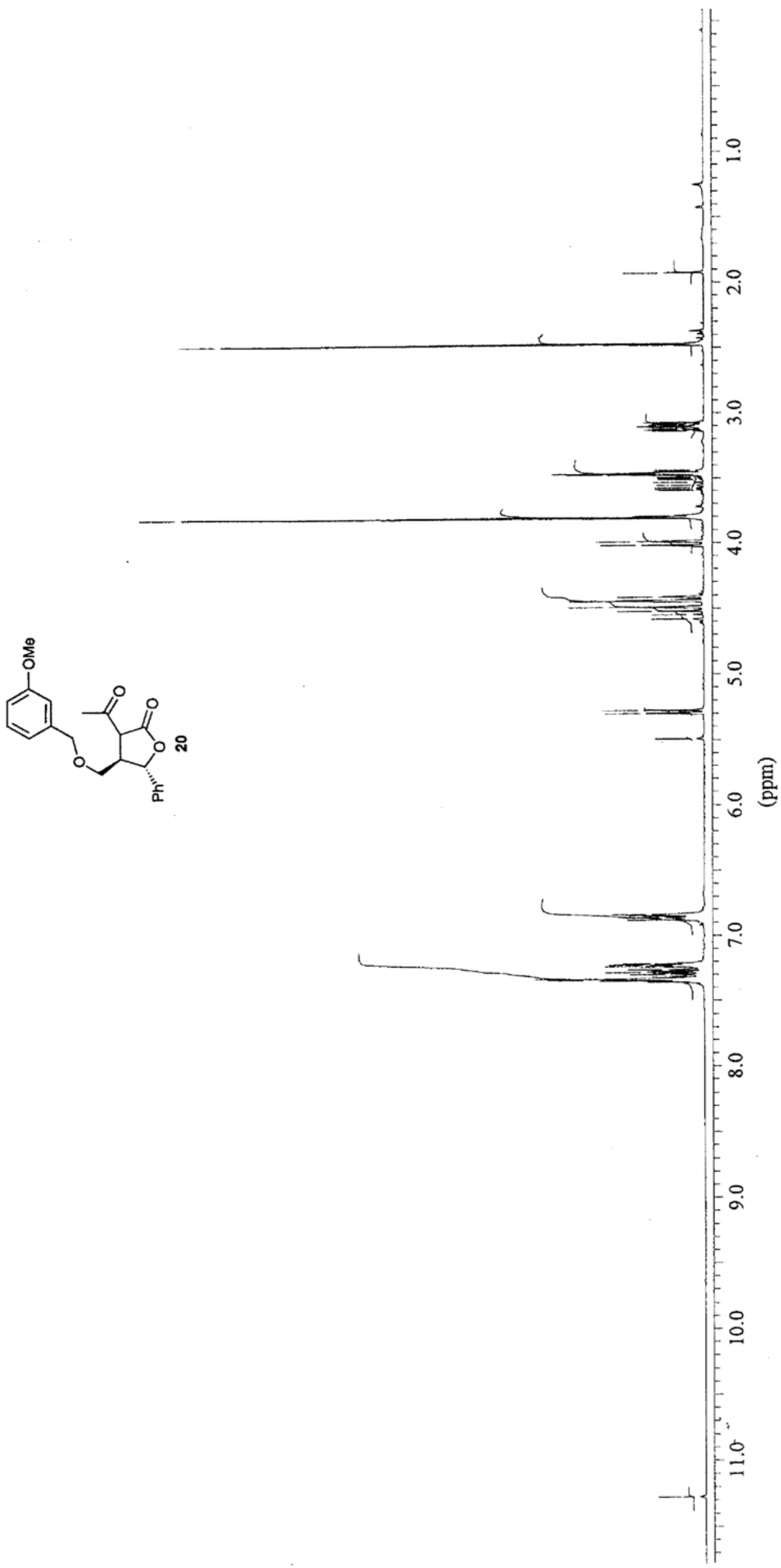



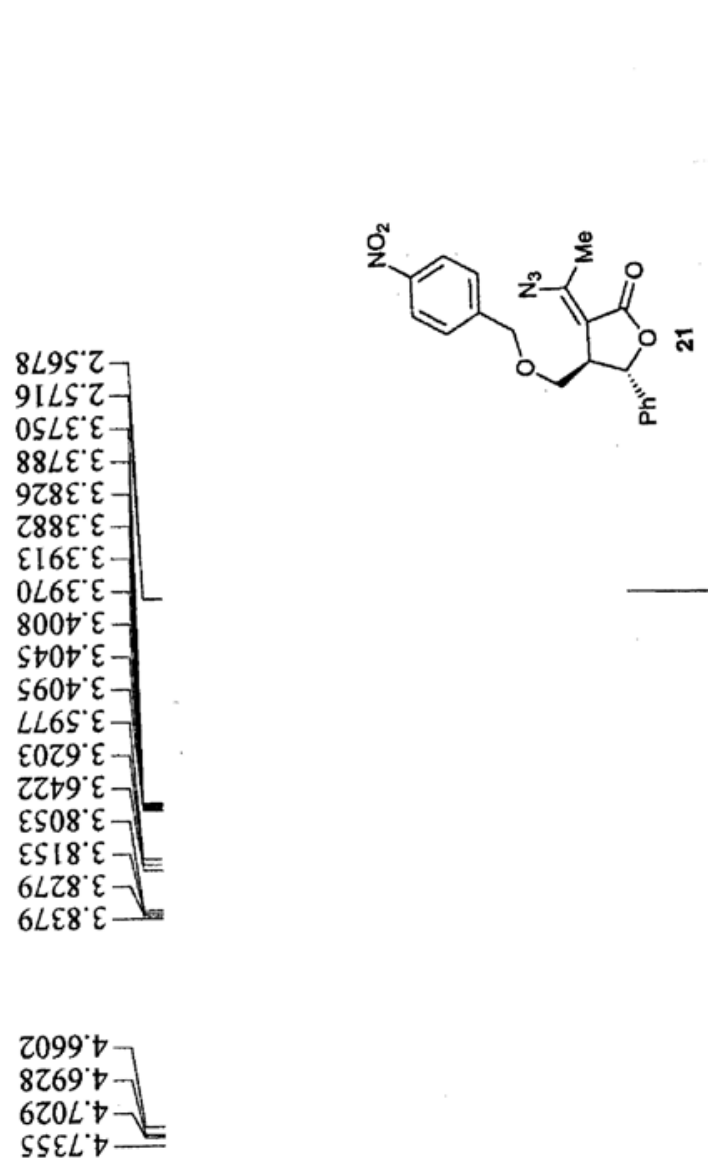

9LOS'S ZEIS'S L

$\downarrow t S Z L L$ $009 C^{\circ} L$ 㞔

E992'L

$\varepsilon I L Z^{\circ} L-$

ISLZ L

$S 68 Z^{\circ} L-$

I $00 \varepsilon^{\circ} L-$

$\varepsilon 80 \varepsilon$ 'L-

9†I $\varepsilon^{\circ} L$

IZZE' $L$

6کZE L

96ZE $L$

$\angle \nabla t \varepsilon^{\circ} L$

I $6 S E^{\circ} L-$

ऽฯ9 $\Sigma^{\circ} L$

09LE $L$ -

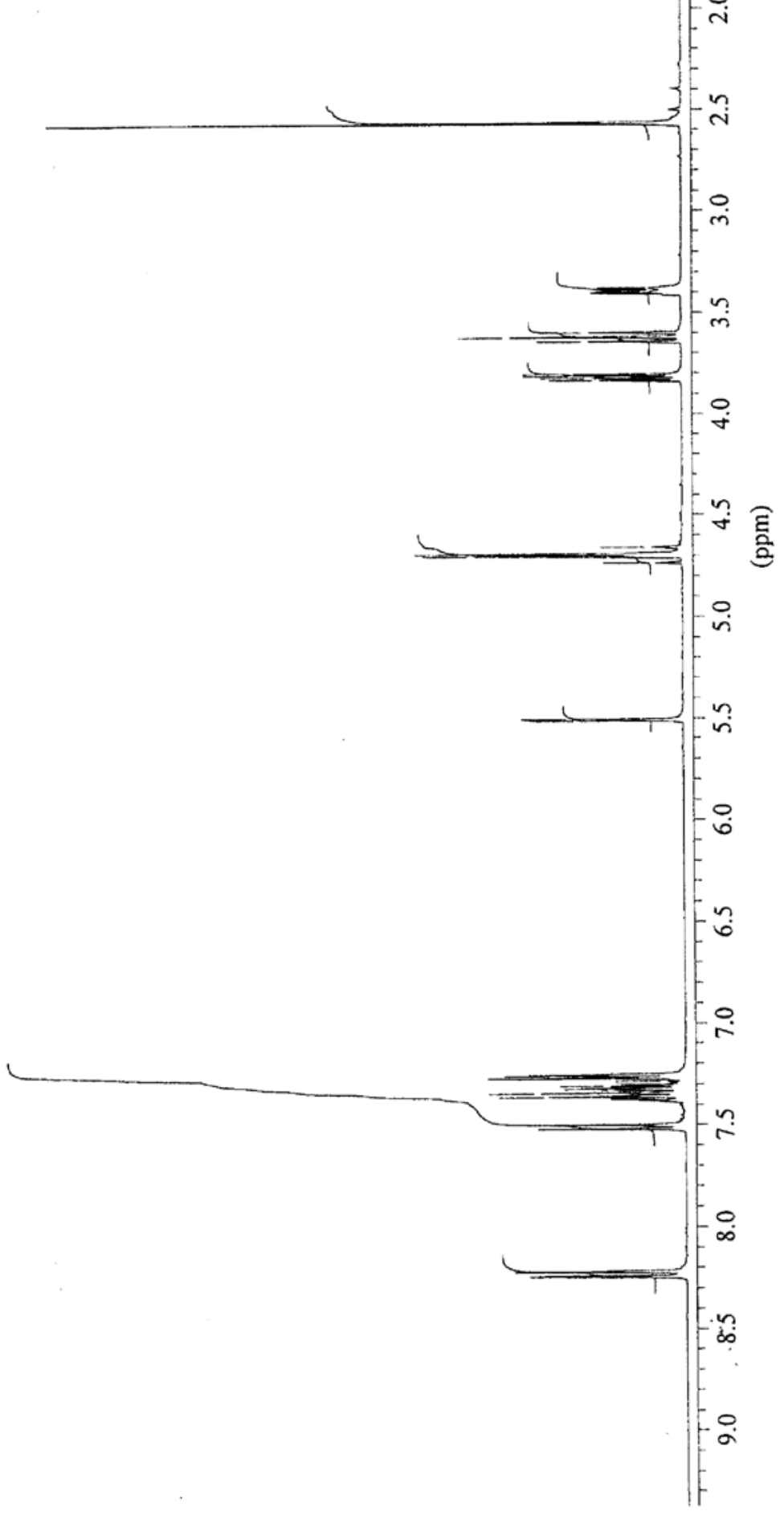



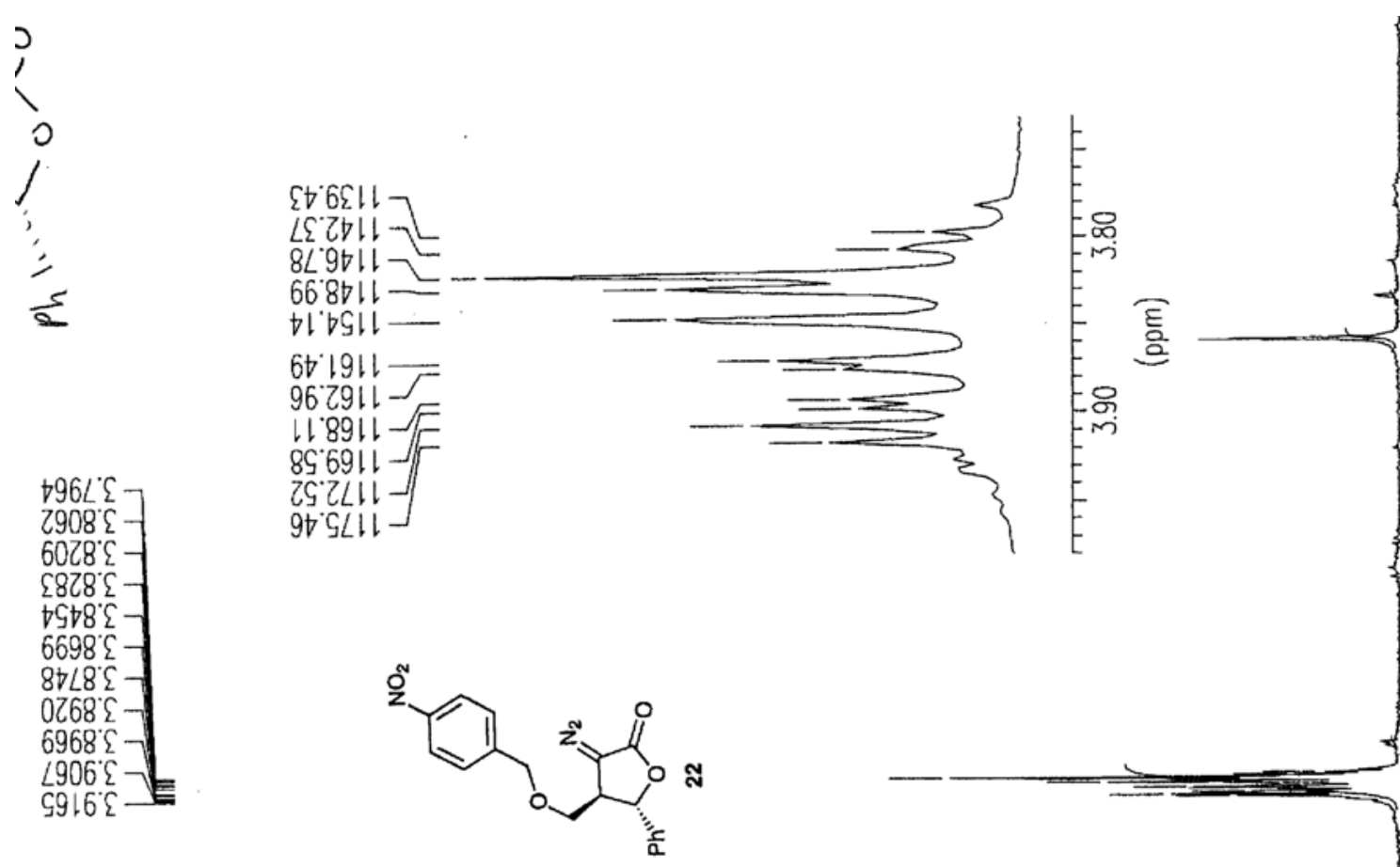

821Lt -

0561 ' 9

$\angle 0025-2$
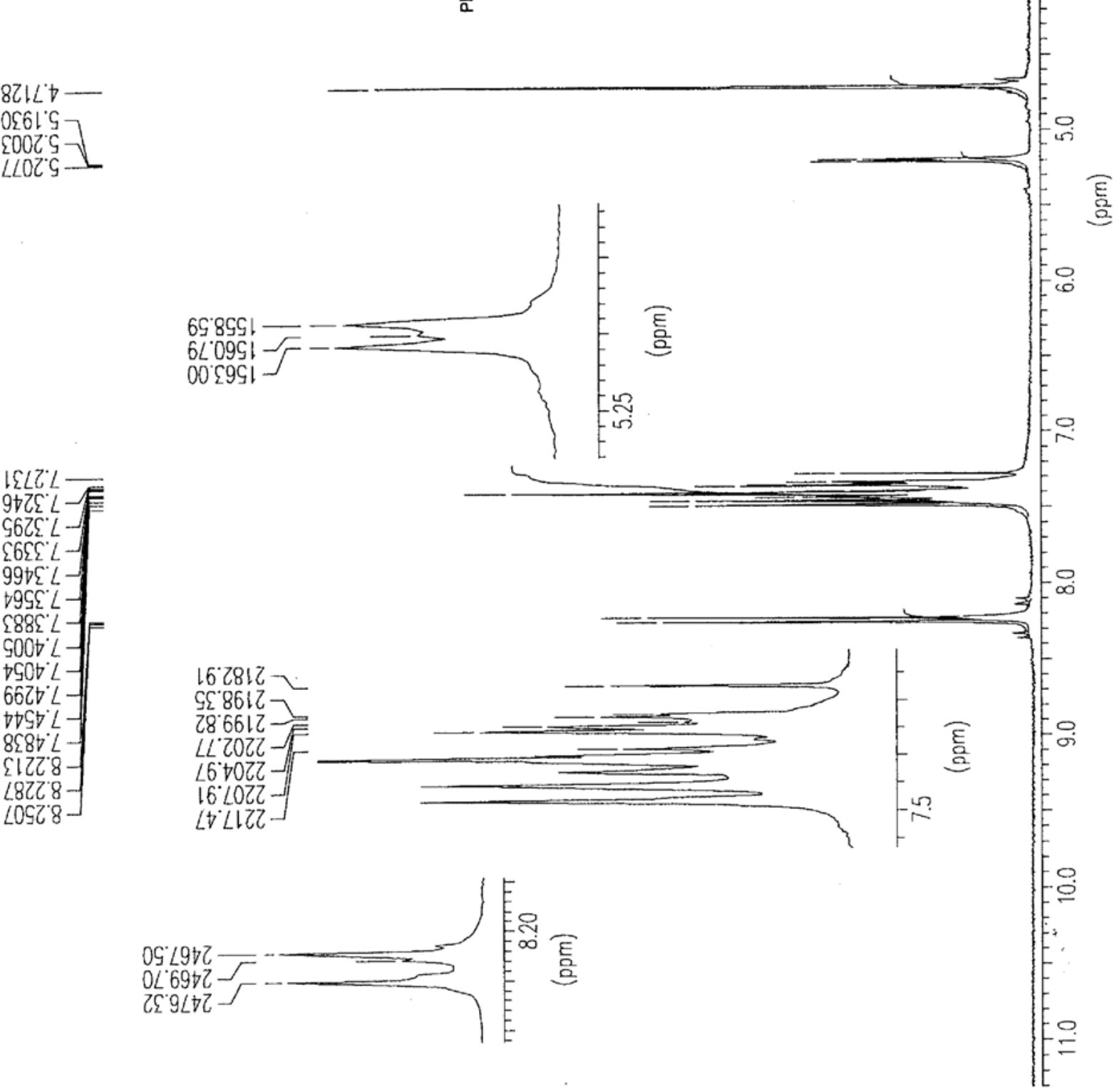
$\angle Z \varepsilon L^{\circ} \varepsilon_{-}$

$\varepsilon \nabla \varepsilon L^{\prime} \varepsilon-$

Z8EL' $\varepsilon-$

$\nabla \nabla \nabla L^{\circ} \mathcal{E}-$

$66 \nabla L^{\circ} \varepsilon$

$0 \varepsilon S L \mathcal{E}-$

9I $9 L^{\circ} \varepsilon$

$\downarrow \mathcal{E} L L^{\prime} \varepsilon-$

$\angle S L L^{\prime} \varepsilon$

ZI $8 L^{\circ} \varepsilon$

$\varsigma Z 18^{\circ} \mathcal{E}$

I IZ8 $\mathcal{E}$

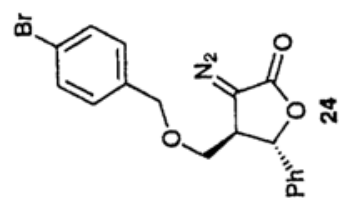

$0 \varepsilon \neg \varsigma^{\circ} \downarrow-$

$9+S I^{\prime} S$

6L9I'S

E99 I' $L$

LE6I' $L-E$

9692 $L=$

$910 \varepsilon^{\circ} L-$

SSOE $L-$

ISZE'L

IZEE'L.

S†8 $\varepsilon^{\circ} L$

$006 \varepsilon^{\circ} L$

I $L 6 \varepsilon^{\circ} L$

$960 t^{\circ} L$

LZIt'L

$\varepsilon 687^{\circ} \mathrm{L}$

$9 S 6 t^{\circ} \mathrm{L}-$

SLIS'L.

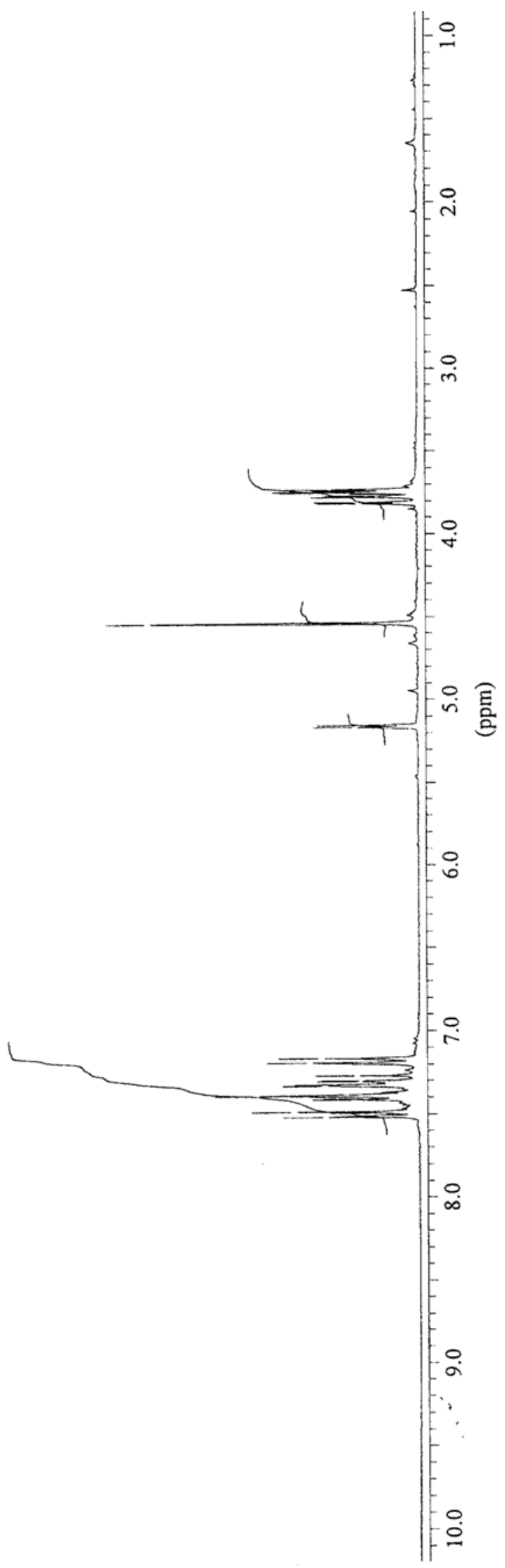



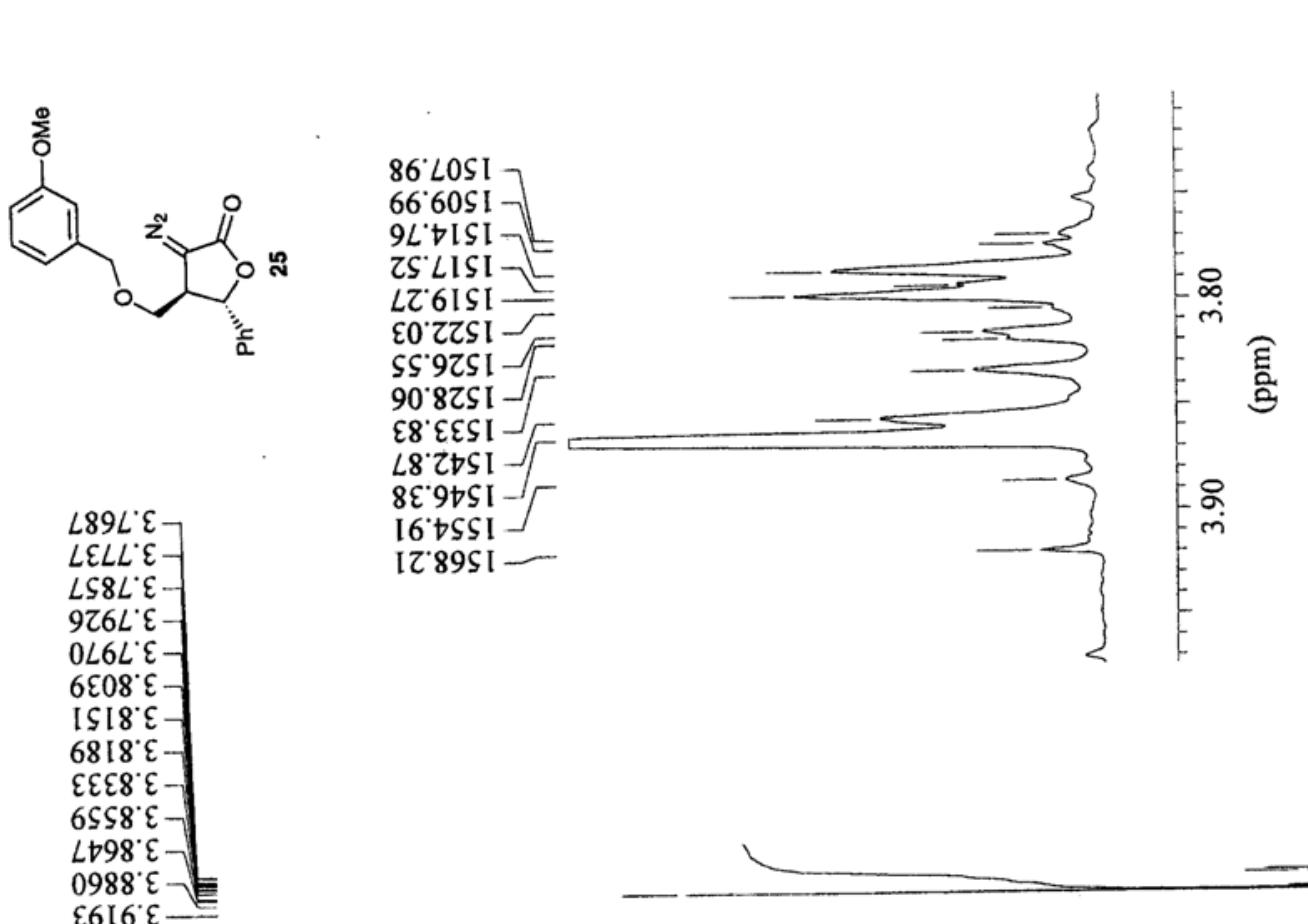

$0819^{\circ} \bullet-$

t602's

l022's

$8606^{\circ} 9$

$\angle 926^{\circ} 9$

6076.9

ZIIEL

OSIE $L$

SLIEL

$8 \varepsilon \varepsilon \varepsilon{ }^{\circ} L$

$69 \varepsilon \varepsilon^{\circ} L$

†I9E'L

I LLE $L$

SI8E'L

SI6E'L

$\angle 66 \varepsilon^{\circ} L$

OIIt'L

ZLIVL $L$

ZZZt L

$9925^{\circ} L$

6ZEt $L$

$\varepsilon L \nabla T^{\circ} L-$

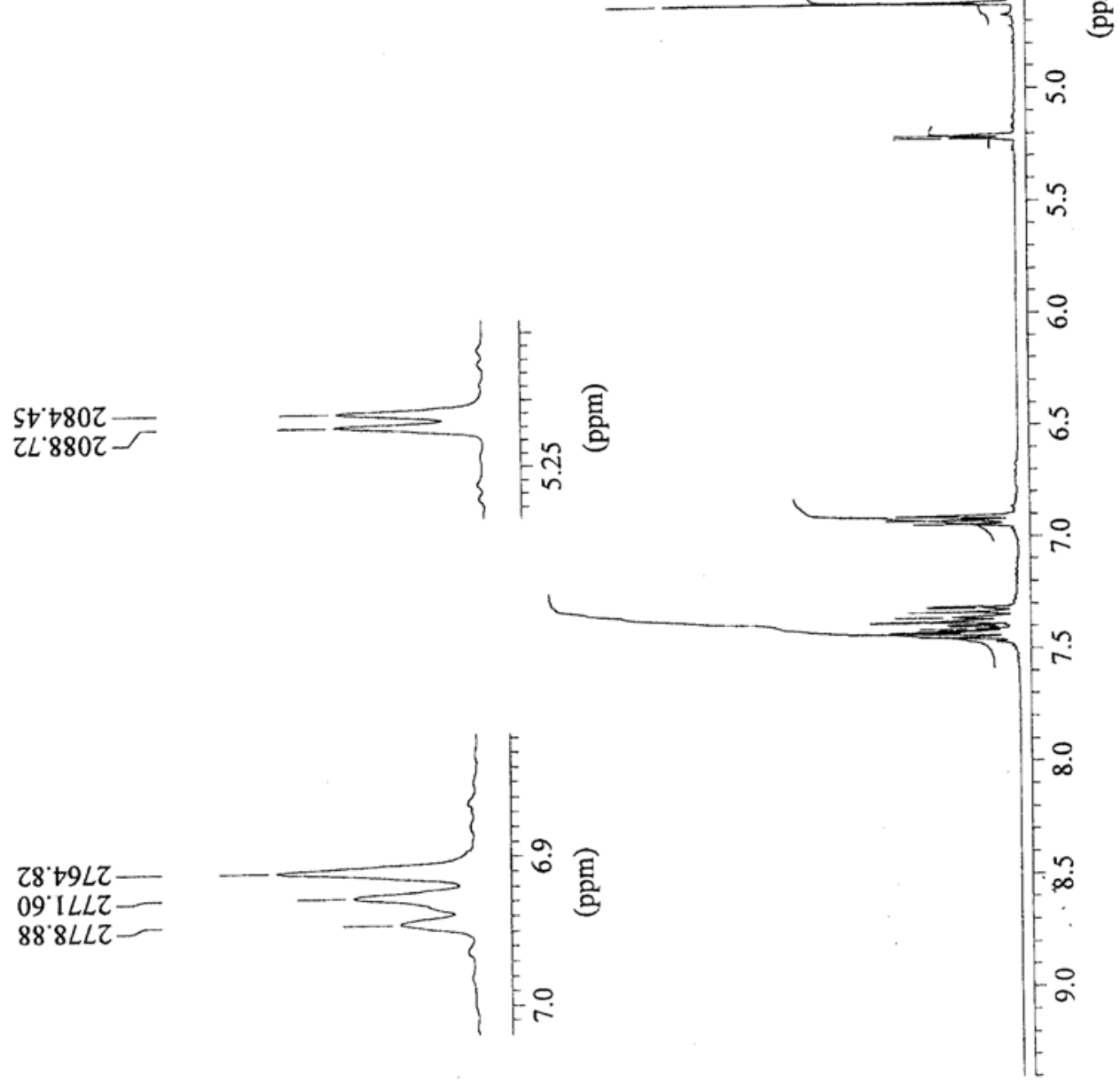



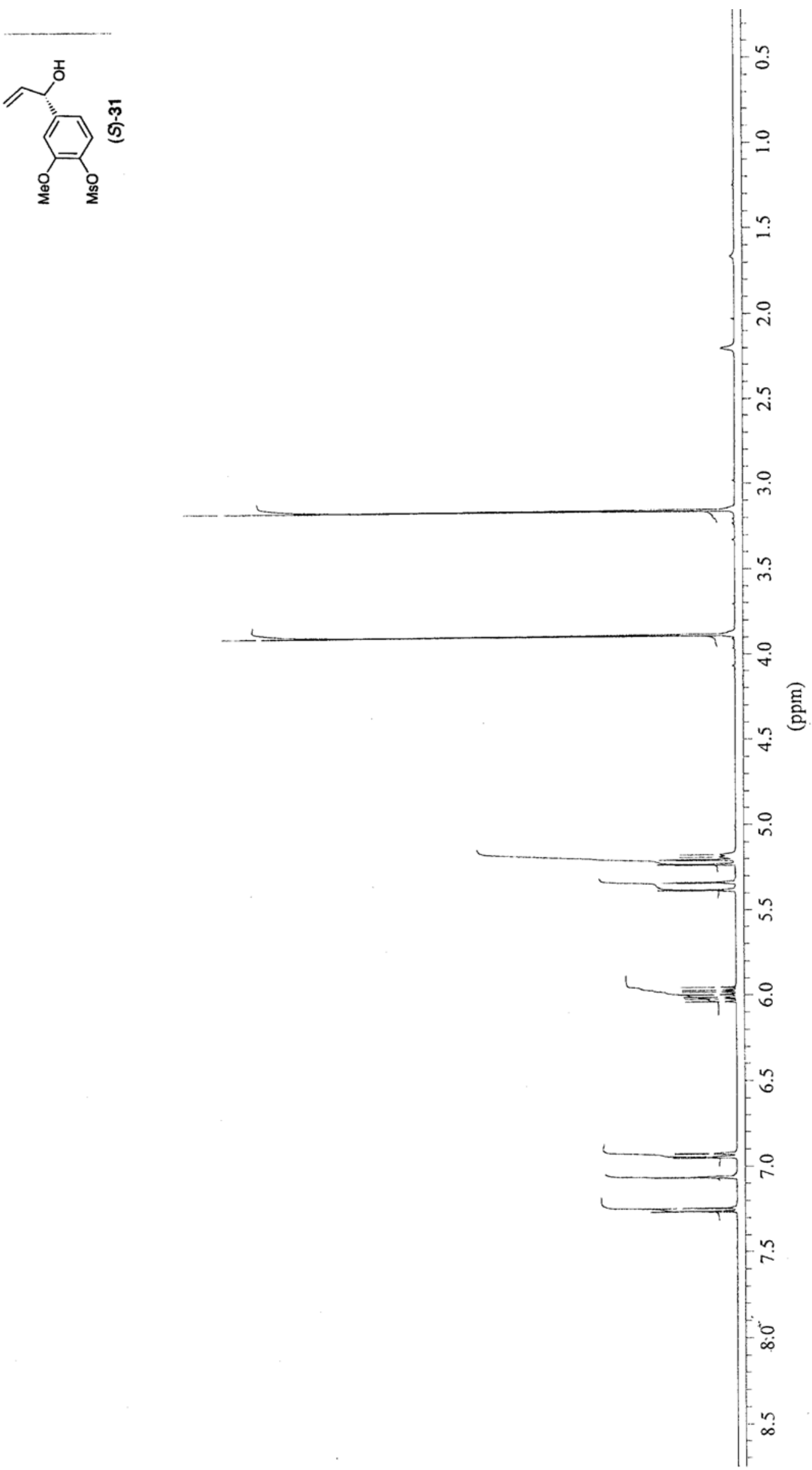

\ES $6^{\circ} \varsigma$

$26 \mathrm{L6} 6^{\circ} \mathrm{S}$

$6066^{\circ} \mathrm{S}$

$2966^{\circ} \varsigma$

$8110^{\circ} 9-$

$6120^{\circ} 9-$

$9 \angle \varepsilon 0^{\circ} 9$

9276.9

9276.9-

9Lt6. $9-$

$S 090^{\circ} \mathrm{L}-$

$6 \nrightarrow 90^{\circ} \mathrm{L}$

$\triangle Z \nabla Z ' L$

$009 Z^{\circ} L$

[E9Z: L - 
S 21
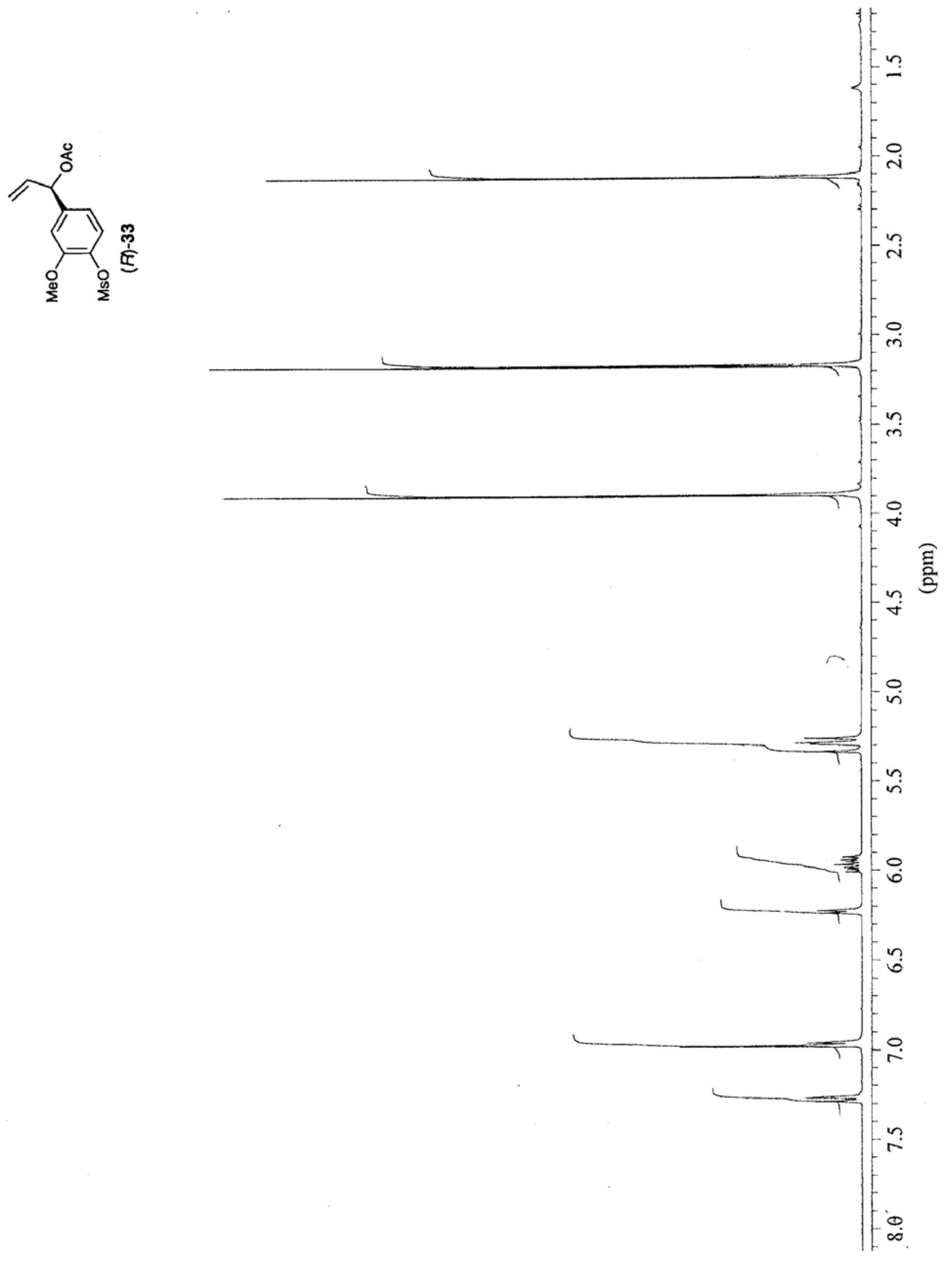


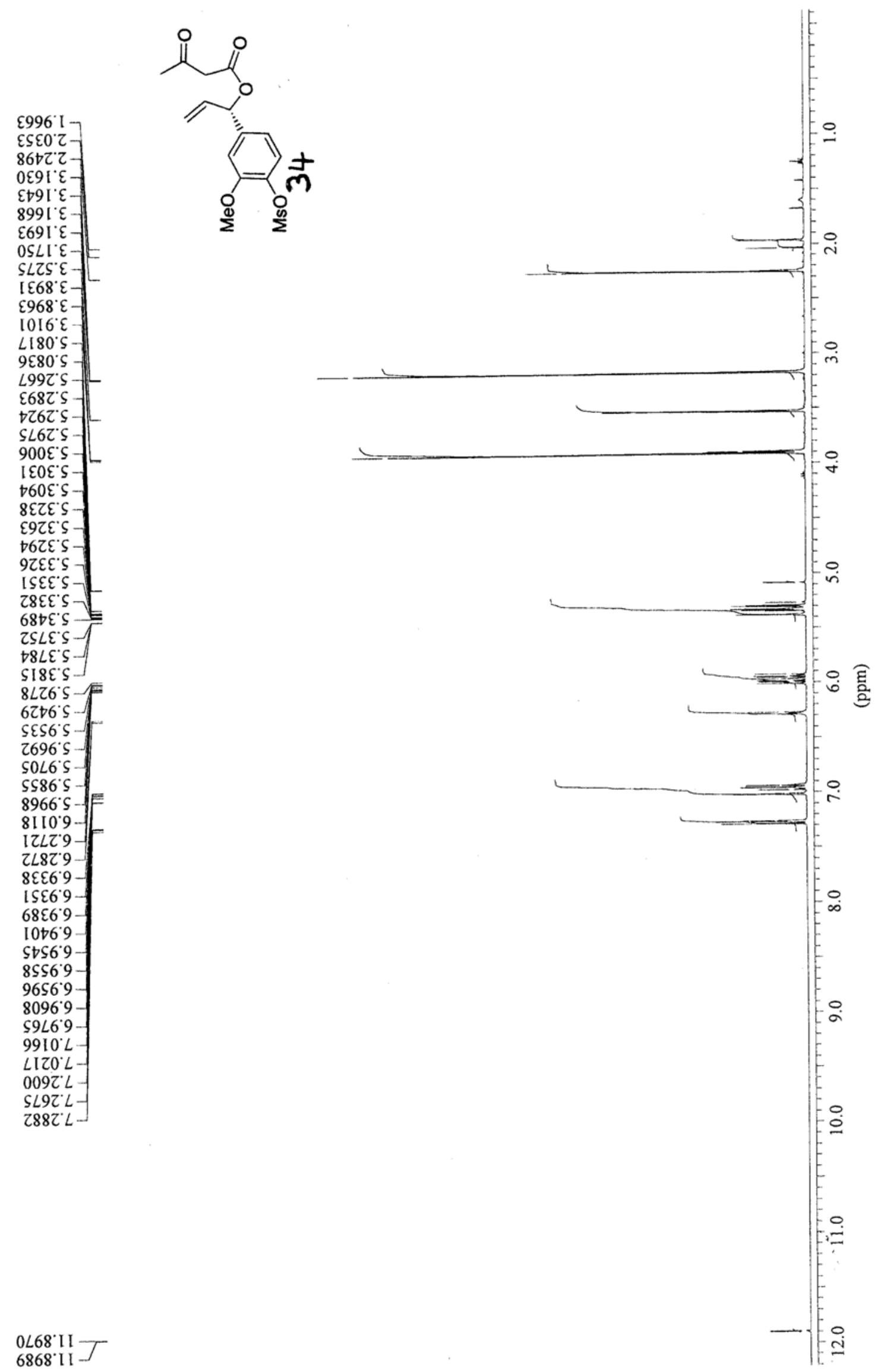




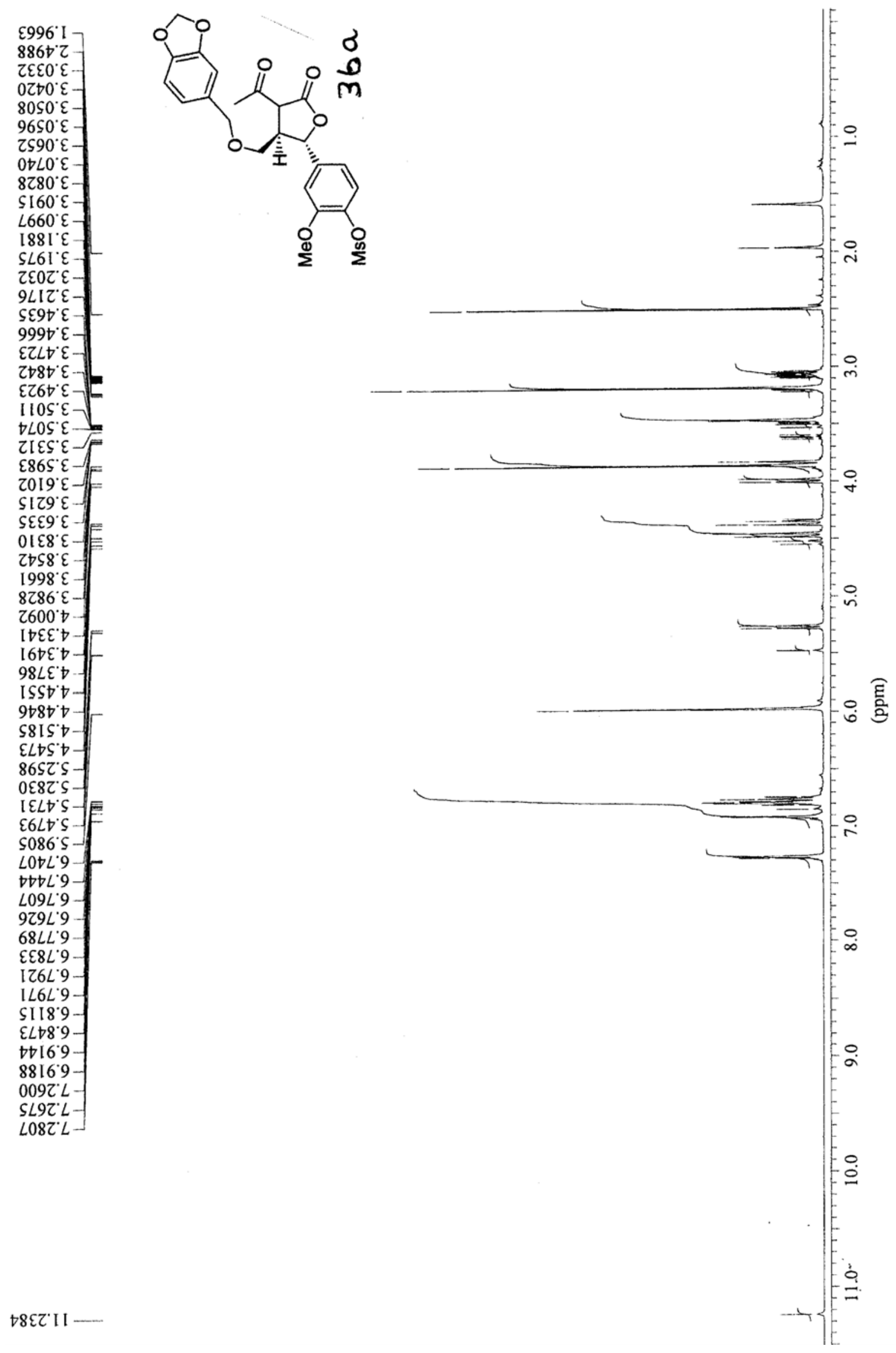



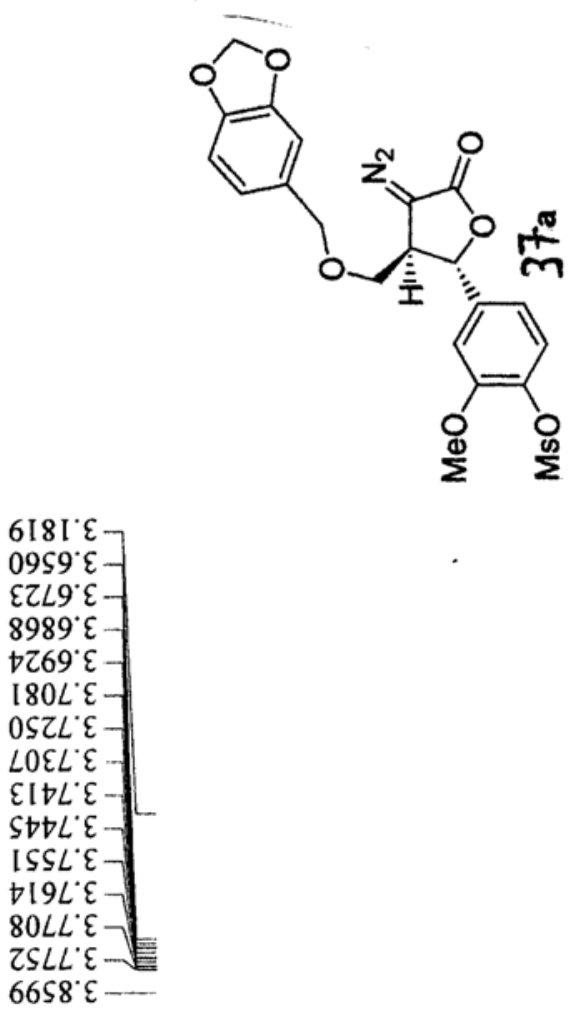

$1280^{\circ} t \ldots$

Zะ9 [' $\mathrm{S}$

ISLI'S

$\angle 196^{\circ} S$
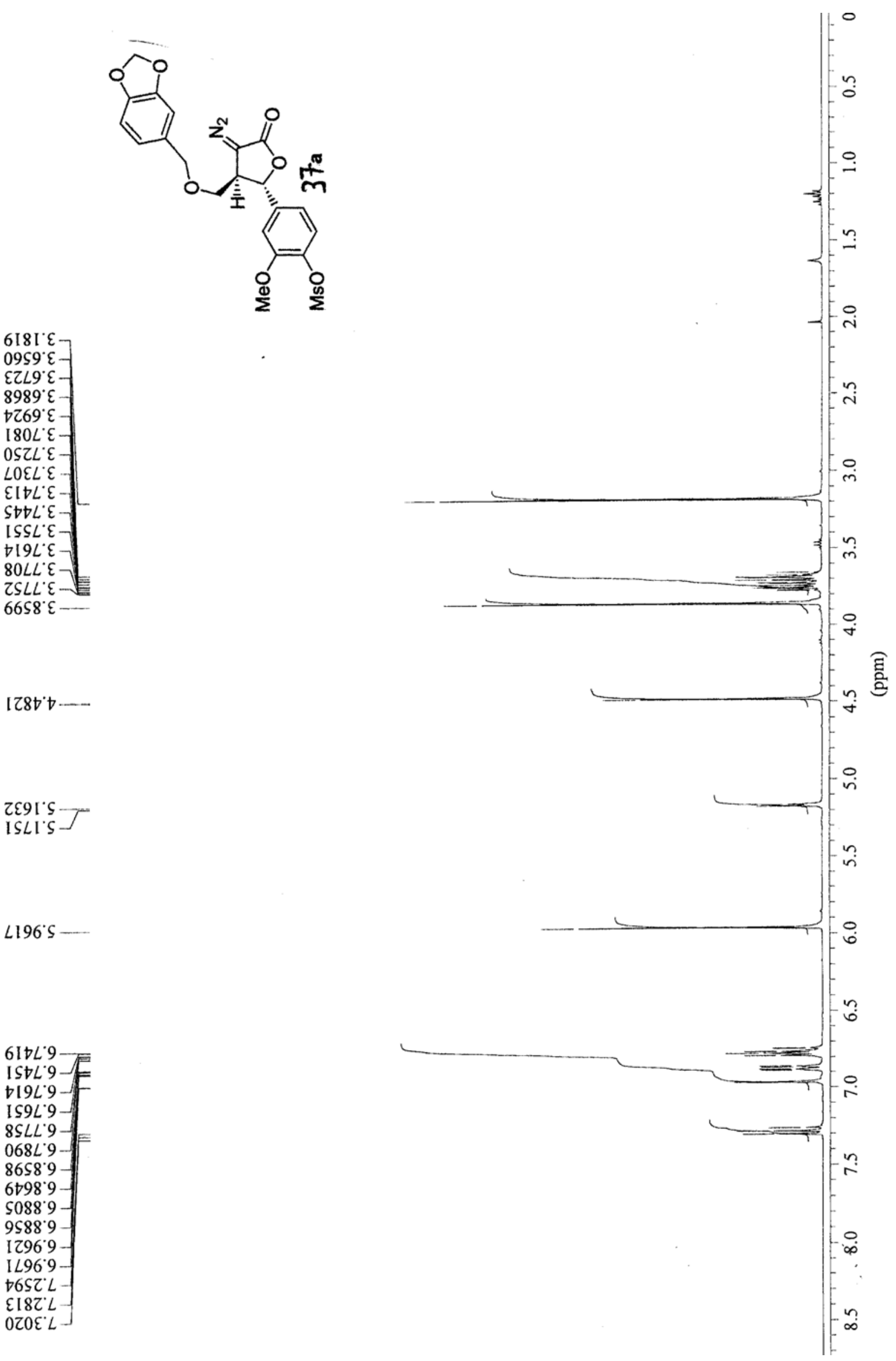

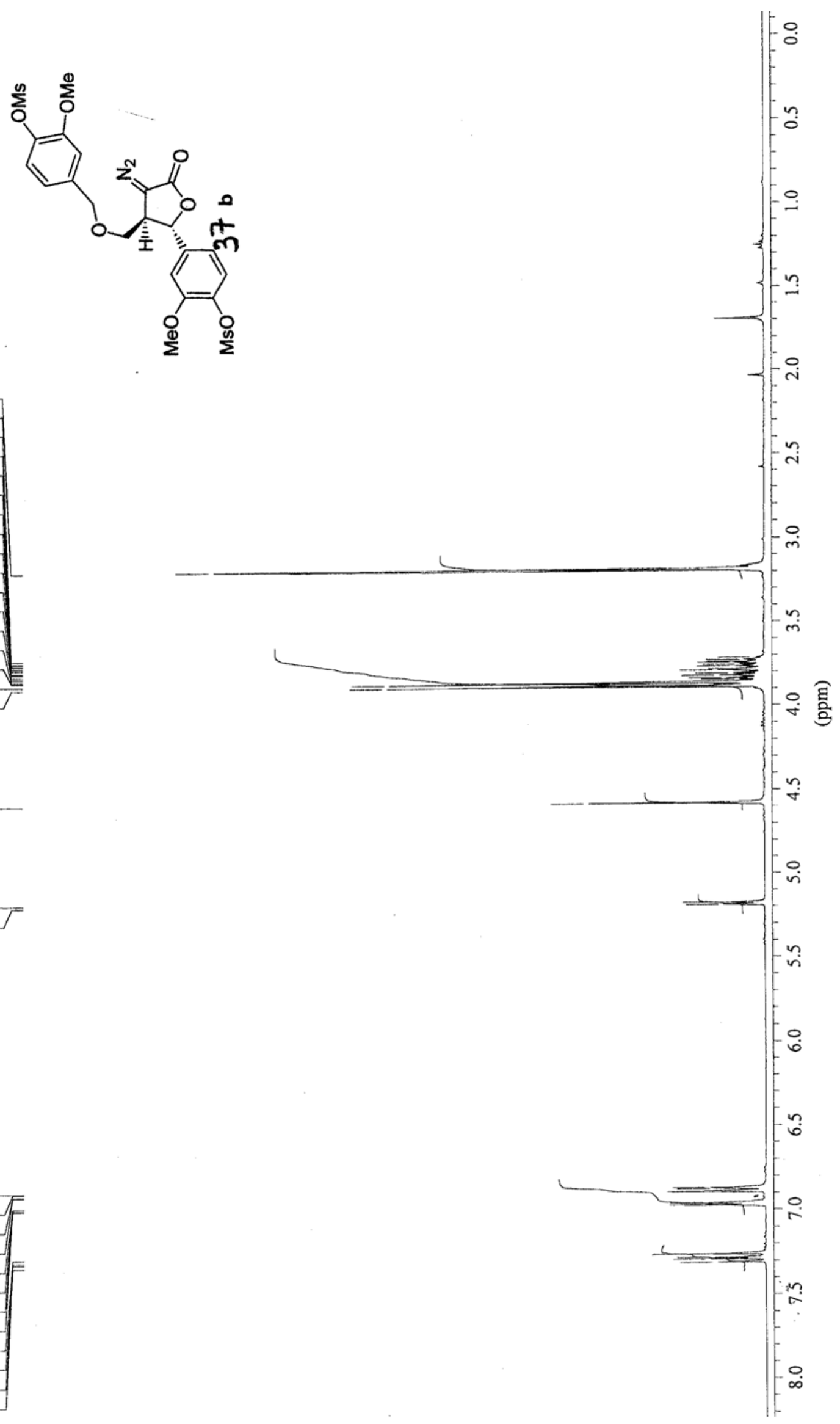
S 26

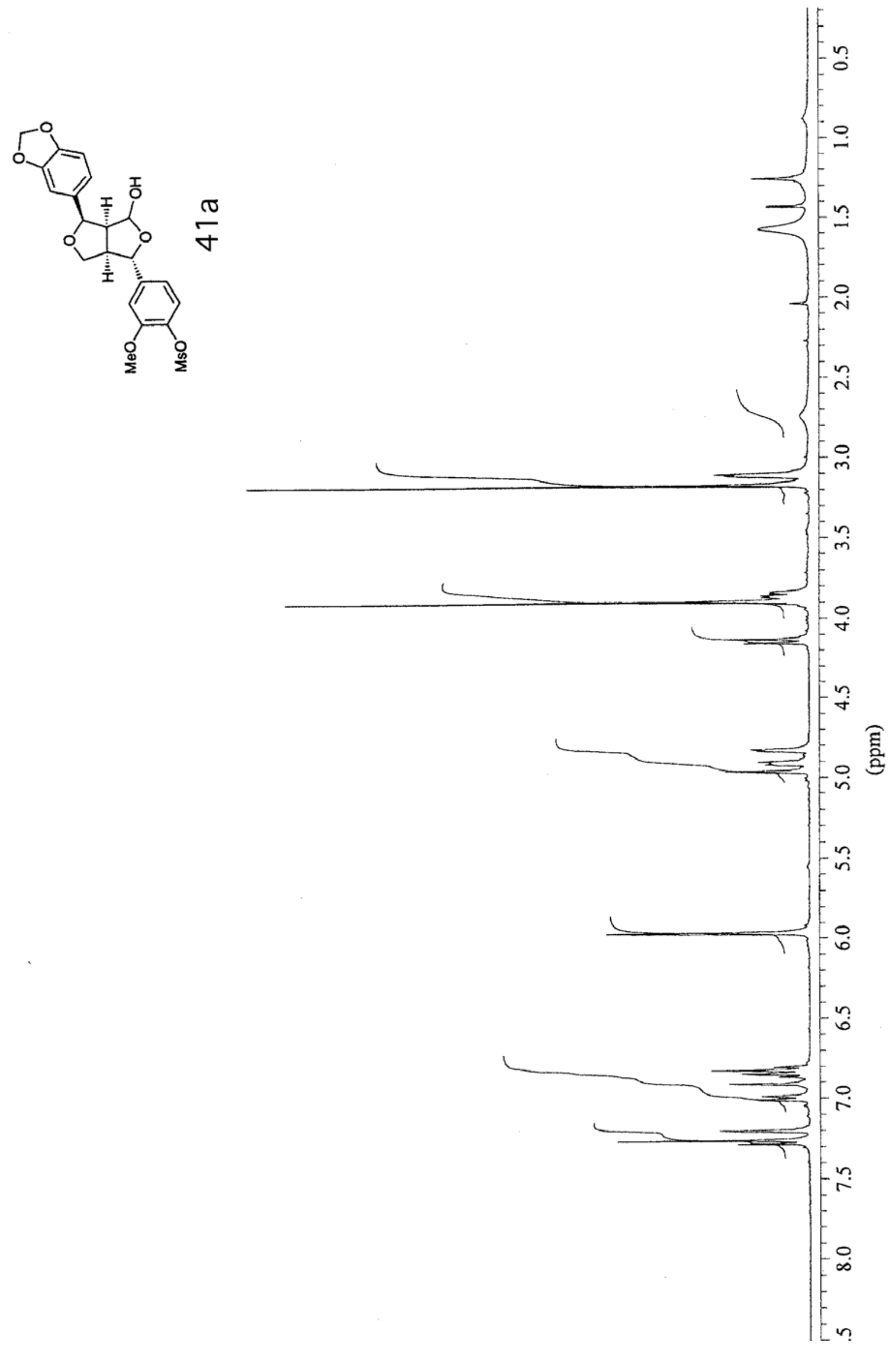




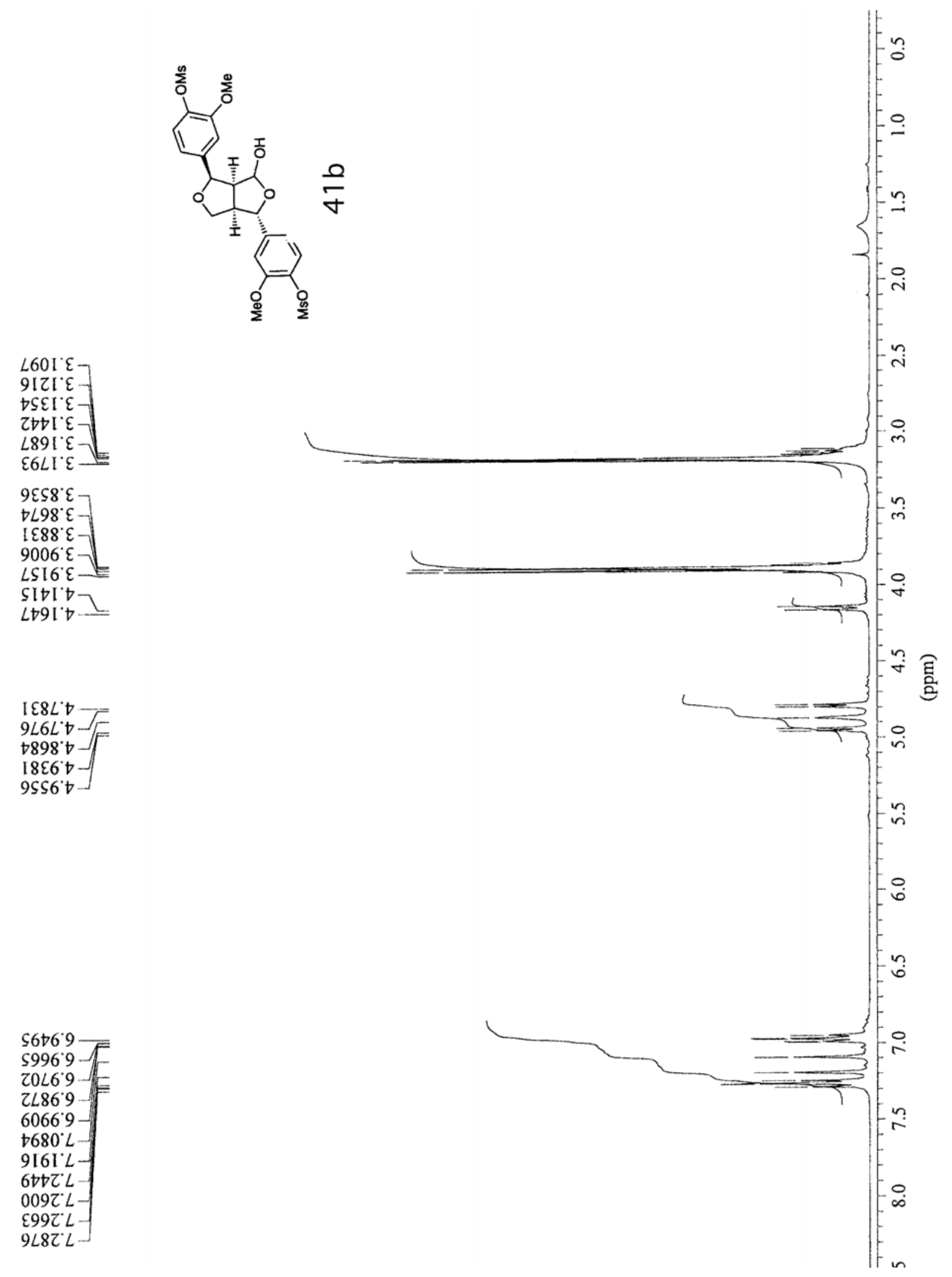




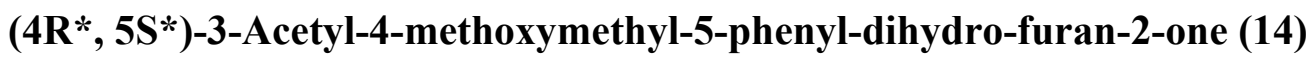<smiles>COC[C@@H]1C(C(C)=O)C(=O)O[C@@H]1c1ccccc1</smiles>

14

\author{
$\mathrm{C}_{14} \mathrm{H}_{16} \mathrm{O}_{4}$ \\ m.w. $=248.28 \mathrm{~g} / \mathrm{mol}$ \\ Colourless oil
}

To a solution of cyclopropane $12(108 \mathrm{mg}, 0.50 \mathrm{mmol})$ in dry $\mathrm{MeOH}(2 \mathrm{~mL})$ was added $\mathrm{Zn}(\mathrm{OTf})_{2}$ $\left(218 \mathrm{mg}, 0.60 \mathrm{mmol}\right.$ ) and the mixture microwaved at $120{ }^{\circ} \mathrm{C}$ for $30 \mathrm{~min}$ (pressure 5 bar) before concentrated in vacuo. Purification was accomplished by flash chromatography on silica gel $(2.3 \mathrm{x}$ 14) eluting with EtOAc/hexane (1:9) to EtOAc/hexane (2:3) in $10 \%$ increment rises (50 mL each). The title compound $14(55 \mathrm{mg}, 0.22 \mathrm{mmol}, 44 \%)$ was isolated as a colourless oil along with methyl enol ether 15 (45 mg, $0.17 \mathrm{mmol}, 34 \%$ ) as a colourless oil. Data for title compound 14. FT-IR $v_{\max }$ (neat) $1770 \mathrm{~s}, 1720 \mathrm{~s}, 1217 \mathrm{~m}, 1165 \mathrm{~s}, 1000 \mathrm{~s} \mathrm{~cm}^{-1}$.

${ }^{1}$ H NMR (400 MHz) $\delta 7.42-7.27(5 \mathrm{H}, \mathrm{m}, \mathrm{PhH}), 5.28(1 \mathrm{H}, \mathrm{d}, J=9.3 \mathrm{~Hz},-\mathrm{CHPh}), 3.97(1 \mathrm{H}, \mathrm{d}, J=$ $10.5 \mathrm{~Hz},-\mathrm{CHCO}-), 3.44-3.37\left(2 \mathrm{H}, \mathrm{m},-\mathrm{CHCH}_{2}-\right), 3.35\left(3 \mathrm{H}, \mathrm{s},-\mathrm{OCH}_{3}\right), 3.12$ $\left(1 \mathrm{H}, \mathrm{ddt}, J=10.5,9.5,3.5 \mathrm{~Hz}-\mathrm{CHCH}_{2}-\right), 2.51\left(3 \mathrm{H}, \mathrm{s},-\mathrm{COCH}_{3}\right)$; keto/enol ratio 8:1 - enolic resonances were observed at $\delta 11.28(1 \mathrm{H}, \mathrm{s}), 5.47(1 \mathrm{H}, \mathrm{d}, J$ $=2.3 \mathrm{~Hz}), 1.98(3 \mathrm{H}, \mathrm{s})$.

${ }^{13} \mathbf{C}$ NMR $(100 \mathrm{MHz}) \delta 200.8(\mathbf{C O}$ ket $), 171.7(\mathbf{C O}$ est $), 137.8\left(\mathbf{C}_{\text {ar }}\right), 129.4\left(\mathbf{C H}_{\text {ar }}\right), 129.2\left(\mathbf{C H}_{\text {ar }}\right), 126.8$ $\left(\mathrm{CH}_{\mathrm{ar}}\right), 81.4(-\mathbf{C H P h}), 68.8\left(-\mathrm{CH}_{2}-\right), 59.5(-\mathbf{C H C O}-), 56.1\left(-\mathrm{OCH}_{3}\right), 46.5(-$ $\left.\mathrm{CHCH}_{2}-\right), 30.5\left(-\mathrm{COCH}_{3}\right)$; enolic resonances were observed at $\delta 170.8$, $140.9,125.4,115.2,82.8,70.4,46.7$.

LRMS (CI, NH $\mathrm{NH}_{3} \quad \mathrm{~m} / \mathrm{z}$ (relative intensity) $249(100)[\mathrm{M}+\mathrm{H}]^{+}, 266(32)\left[\mathrm{M}+\mathrm{NH}_{4}\right]^{+}$.

HRMS (EI) $\quad$ Calcd for $\mathrm{C}_{14} \mathrm{H}_{16} \mathrm{O}_{4} 248.1049$, found 248.1054. 


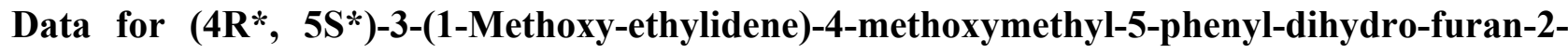
one (15)

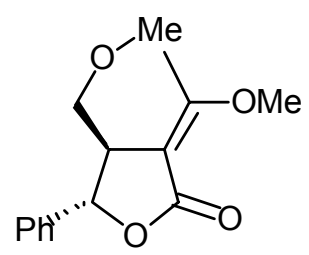

15

\author{
$\mathrm{C}_{15} \mathrm{H}_{18} \mathrm{O}_{4}$ \\ $\mathrm{m} . \mathrm{w} .=262.30 \mathrm{~g} / \mathrm{mol}$ \\ Colourless oil
}

FT-IR $v_{\max }$ (neat) $1701 \mathrm{~s}, 1644 \mathrm{~s}, 1437 \mathrm{~m}, 1382 \mathrm{~m}, 1217 \mathrm{~s}, 1117 \mathrm{~s}, 1090 \mathrm{~s} \mathrm{~cm}^{-1}$.

${ }^{1}$ H NMR (400 MHz) $\delta$ 7.38-7.28 (5H, m, PhH), $5.53(1 \mathrm{H}, \mathrm{d}, J=4.5 \mathrm{~Hz},-\mathrm{CHPh}), 3.70(3 \mathrm{H}, \mathrm{s},-$ $\left.\mathrm{C}=\mathrm{COCH}_{3}\right), 3.70(1 \mathrm{H}$, obsc dd, $J=9.0,3.5 \mathrm{~Hz}$, -СНCHН-), 3.49 $(1 \mathrm{H}, \mathrm{t}, J=9.0 \mathrm{~Hz},-\mathrm{CHCHH}-), 3.40(3 \mathrm{H}, \mathrm{s}$, $-\mathrm{CH}_{2} \mathrm{OCH}$ ), 3.42-3.34 $\left(1 \mathrm{H}, \mathrm{m},-\mathrm{CHCH}_{2^{-}}\right), 2.33\left(3 \mathrm{H}, \mathrm{s},-\mathrm{CCH}_{3}\right)$.

${ }^{13} \mathbf{C}$ NMR $(100 \mathrm{MHz}) \delta 170.1\left(\mathbf{C O}_{\text {est }}\right), 166.5(-\mathrm{C}=\mathbf{C O}-), 142.0\left(\mathbf{C}_{\mathrm{ar}}\right), 129.0\left(\mathbf{C H}_{\mathrm{ar}}\right), 128.3\left(\mathbf{C H}_{\mathrm{ar}}\right)$, $125.6\left(\mathrm{CH}_{\mathrm{ar}}\right), 102.0(-\mathbf{C}=\mathrm{CO}-), 86.9(-\mathbf{C H P h}), 74.2\left(-\mathrm{CH}_{2}-\right), 59.3\left(-\mathrm{CHCH}_{2^{-}}\right)$, $51.9\left(-\mathrm{OCH}_{3}\right), 51.2\left(-\mathrm{OCH}_{3}\right), 14.9\left(-\mathrm{CCH}_{3}\right)$.

LRMS $\left(\mathrm{CI}, \mathrm{NH}_{3}\right) \quad \mathrm{m} / z$ (relative intensity) $263(100)[\mathrm{M}+\mathrm{H}]^{+}, 231$ (64) $[\mathrm{M}+\mathrm{H}$ $\mathrm{MeOH})]^{+}$.

HRMS $(E S+v e) \quad$ Calcd for $\mathrm{C}_{15} \mathrm{H}_{18} \mathrm{O}_{4} \mathrm{Na} 285.1097$, found 285.1095. 


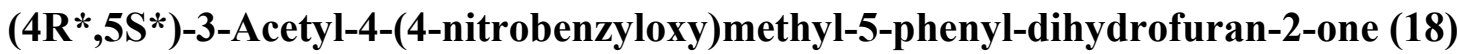

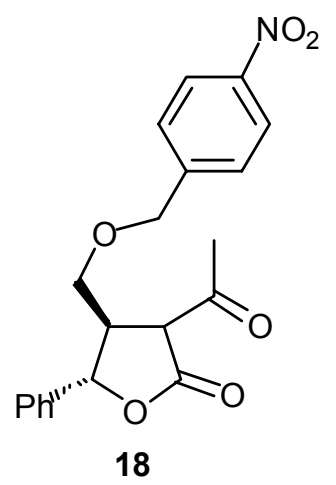

\author{
$\mathrm{C}_{20} \mathrm{H}_{19} \mathrm{NO}_{6}$ \\ m.w. $=369.37 \mathrm{~g} / \mathrm{mol}$ \\ White powdery solid
}

The title compound was prepared according to the method outlined for 16, whereby reaction of cyclopropane $12(216 \mathrm{mg}, 1.0 \mathrm{mmol})$ with 4-nitrobenzylalcohol (459 mg, $3.0 \mathrm{mmol})$ and workup under the conditions described gave a crude viscous yellow oil $(970 \mathrm{mg})$. The excess benzyl alcohol was removed by distillation under reduced pressure (b.p. $\sim 200{ }^{\circ} \mathrm{C}, 0.5$ mbar) and the residue purified by flash chromatography on silica gel $(2.3 \times 8)$ eluting with EtOAc/hexane (1:9) to EtOAc/hexane (1:1) in $10 \%$ increment rises (40 mL each) to yield the title compound 18 (326 mg, $0.88 \mathrm{mmol}, 88 \%$ ) as a viscous colourless oil that solidified on standing to provide a white powdery solid.

MP 79-81 ${ }^{\circ} \mathrm{C}($ EtOAc/hexane).

FT-IR $v_{\max }$ (neat) $1768 \mathrm{~s}, 1718 \mathrm{~s}, 1512 \mathrm{~s}, 1344 \mathrm{~s}, 1156 \mathrm{~s}, 1123 \mathrm{~s}, 1088 \mathrm{~s} \mathrm{~cm}^{-1}$.

${ }^{1}$ H NMR $(400 \mathrm{MHz}) \delta 8.20(2 \mathrm{H}, \mathrm{d}, J=8.5 \mathrm{~Hz}, \operatorname{ArH}), 7.43(2 \mathrm{H}, \mathrm{d}, J=8.5 \mathrm{~Hz}, \mathrm{ArH}), 7.43-7.28$ $(5 \mathrm{H}, \mathrm{m}, \mathrm{PhH}), 5.30(1 \mathrm{H}, \mathrm{d}, J=9.3 \mathrm{~Hz},-\mathrm{CHPh}), 4.62(1 \mathrm{H}, \mathrm{d}, J=13.1 \mathrm{~Hz},-$ OCHHAr), $4.57(1 \mathrm{H}, \mathrm{d}, J=12.8 \mathrm{~Hz}, \quad$-OCHHAr $), 3.97(1 \mathrm{H}, \mathrm{d}, J=$ $10.5 \mathrm{~Hz},-\mathrm{CHCO}-), 3.61$ (1H, dd, $J=10.0,4.0 \mathrm{~Hz},-\mathrm{CHCHH-),} 3.57$ (1H, dd, $J=10.0,4.0 \mathrm{~Hz}, \quad$-CHCHH- $), 3.23(1 \mathrm{H}, \mathrm{ddt}, J=10.3,9.3,4.0 \mathrm{~Hz}-$ $\left.\mathrm{CHCH}_{2-}\right), 2.51(3 \mathrm{H}, \mathrm{s},-\mathrm{COCH} 3)$; keto/enol ratio 18:1 - enolic resonances were observed at $\delta 1.89(3 \mathrm{H}, \mathrm{s})$.

${ }^{13} \mathbf{C}$ NMR $(100 \mathrm{MHz}) \delta 200.5\left(\mathbf{C O}_{\text {ket }}\right), 171.4\left(\mathbf{C O}_{\text {est }}\right), 148.0\left(\mathbf{C}_{\mathrm{ar}}\right), 145.3\left(\mathbf{C}_{\mathrm{ar}}\right), 137.5\left(\mathbf{C}_{\mathrm{ar}}\right), 129.6$ $\left(\mathbf{C H}_{\mathrm{ar}}\right), 129.3\left(\mathrm{CH}_{\mathrm{ar}}\right), 128.1\left(\mathbf{C H}_{\mathrm{ar}}\right), 126.8\left(\mathbf{C H}_{\mathrm{ar}}\right), 124.2\left(\mathbf{C H}_{\mathrm{ar}}\right), 87.2(-$ $\mathrm{CHPh}), 72.4\left(-\mathrm{CH}_{2} \mathrm{Ar}\right), 67.6\left(-\mathrm{CHCH}_{2} \mathrm{O}-\right), 56.3 \quad$ (-CHCO-), 46.3 ($\left.\mathrm{CHCH}_{2}-\right), 30.4\left(-\mathrm{COCH}_{3}\right)$; enolic resonances were observed at $\delta 87.2,62.5$, 59.1, 51.0, 21.1.

LRMS (ES +ve) $\quad m / z$ (relative intensity) $761(100)[2 \mathrm{M}+\mathrm{Na}]^{+}, 1130(50)[3 \mathrm{M}+\mathrm{Na}]^{+}$.

CHN Anal. Calcd for $\mathrm{C}_{20} \mathrm{H}_{19} \mathrm{NO}_{6}$ : C, 65.03; H, 5.18; N, 3.79. Found: C, 65.22; H, 5.21; N, 3.77 . 


\title{
(4R*, 5S*)-3-Acetyl-4-(4-bromobenzyloxy)methyl-5-phenyl-dihydrofuran-2-one (19)
}

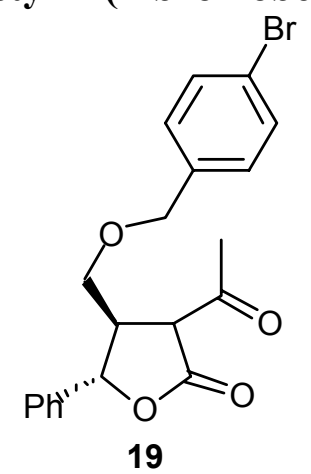

\author{
$\mathrm{C}_{20} \mathrm{H}_{19} \mathrm{O}_{4} \mathrm{Br}$ \\ m.w. $=403.27 \mathrm{~g} / \mathrm{mol}$
}

White powdery solid

The title compound was prepared according to the method outlined for $\mathbf{1 6}$, whereby reaction of cyclopropane $12(432 \mathrm{mg}, 2.0 \mathrm{mmol})$ with 4-bromobenzylalcohol (1.12 g, $6.0 \mathrm{mmol})$ and workup under the conditions described gave a crude viscous yellow oil (1.62 g). The excess benzyl alcohol was removed by distillation under reduced pressure (b.p. $\sim 170{ }^{\circ} \mathrm{C}, 0.5 \mathrm{mbar}$ ) and the residue purified by flash chromatography on silica gel $(2.7$ x 8) eluting with EtOAc/hexane (1:9) to EtOAc/hexane (3:7) in $10 \%$ increment rises $(100 \mathrm{~mL}$ each) to yield the title compound 19 (732 $\mathrm{mg}, 1.82 \mathrm{mmol}, 91 \%$ ) as a viscous colourless oil that solidified on standing to provide a white powdery solid.

MP

FT-IR $v_{\text {max }}$ (neat)

${ }^{1}$ H NMR (400 MHz) $\delta 7.50(2 \mathrm{H}, \mathrm{d}, J=8.3 \mathrm{~Hz}, \operatorname{ArH}), 7.38-7.21(5 \mathrm{H}, \mathrm{m}, \mathrm{PhH}), 7.16(2 \mathrm{H}, \mathrm{d}, J=8.3$ $\mathrm{Hz}, \mathrm{ArH}), 5.27(1 \mathrm{H}, \mathrm{d}, J=9.5 \mathrm{~Hz},-\mathrm{CHPh}), 4.49(1 \mathrm{H}, \mathrm{d}, J=12.1 \mathrm{~Hz},-$ OCHHAr), $4.40(1 \mathrm{H}, \mathrm{d}, J=12.1 \mathrm{~Hz},-$ OCHHAr $), 3.97(1 \mathrm{H}, \mathrm{d}, J=10.6 \mathrm{~Hz}$, CHCO-), 3.50 (1H, dd, $J=10.0,3.5 \mathrm{~Hz},-\mathrm{CHCHH}-), 3.47$ (1H, dd, $J=10.0$, $3.5 \mathrm{~Hz},-\mathrm{CHCHH}-), 3.14\left(1 \mathrm{H}, \mathrm{ddt}, J=10.5,9.5,3.5 \mathrm{~Hz}-\mathrm{CHCH}_{2}-\right), 2.50(3 \mathrm{H}$, $\left.\mathrm{s},-\mathrm{COCH}_{3}\right)$; keto/enol ratio 10:1 - enolic resonances were observed at $\delta$ $11.28(1 \mathrm{H}, \mathrm{s}), 5.48(1 \mathrm{H}, \mathrm{d}, J=2.3 \mathrm{~Hz}), 4.55(1 \mathrm{H}, \mathrm{d}, J=7.5 \mathrm{~Hz}), 1.94(3 \mathrm{H}, \mathrm{s})$.

${ }^{13} \mathbf{C}$ NMR $(100 \mathrm{MHz}) \delta 200.7\left(\mathbf{C O}_{\text {ket }}\right), 171.5\left(\mathbf{C O}_{\text {est }}\right), 137.6\left(\mathbf{C}_{\mathrm{ar}}\right), 136.8\left(\mathbf{C}_{\mathrm{ar}}\right), 132.1\left(\mathbf{C H}_{\mathrm{ar}}\right), 129.9$ $\left(\mathbf{C H}_{\mathrm{ar}}\right), 129.5\left(\mathbf{C H}_{\mathrm{ar}}\right), 129.3\left(\mathbf{C H}_{\mathrm{ar}}\right), 126.7\left(\mathbf{C H}_{\mathrm{ar}}\right), 122.4(\mathbf{\mathbf { C }} \mathrm{ar}), 81.5(-\mathbf{C H P h})$, $72.9\left(-\mathrm{CH}_{2} \mathrm{Ar}\right), 66.1$ (- $\left.\mathrm{CHCH}_{2} \mathrm{O}-\right), 56.1$ (-CHCO-), $46.5\left(-\mathrm{CHCH}_{2}-\right), 30.5$ ($\mathrm{COCH}_{3}$ ); enolic resonances were observed at $\delta 128.7,125.3,82.8,72.3$, 46.8, 19.6.

CHN Anal. $\quad$ Caled for $\mathrm{C}_{20} \mathrm{H}_{19} \mathrm{O}_{4} \mathrm{Br}$ : C, 59.57; H, 4.75. Found: C, 59.54; H, 4.76. 


\section{(4R*, 5S*)-3-Acetyl-4-(3-methoxybenzyloxy)methyl-5-phenyl-dihydro-furan-2-one (20)}<smiles>COc1cccc(COC[C@@H]2C(C(C)=O)C(=O)O[C@H]2c2ccccc2)c1</smiles>

$$
\begin{gathered}
\mathrm{C}_{21} \mathrm{H}_{22} \mathrm{O}_{5} \\
\text { m.w. }=354.40 \mathrm{~g} / \mathrm{mol}
\end{gathered}
$$

Colourless oil

The title compound was prepared according to the method outlined for 16, whereby reaction of cyclopropane 12 (216 mg, $1.0 \mathrm{mmol})$ with 3-methoxybenzylalcohol $(0.37 \mathrm{~mL}, 3.0 \mathrm{mmol})$ and workup under the conditions described gave a crude viscous yellow oil $(705 \mathrm{mg})$. The excess benzyl alcohol was removed by distillation under reduced pressure (b.p. $\sim 130{ }^{\circ} \mathrm{C}, 0.5 \mathrm{mbar}$ ) and the residue purified by flash chromatography on silica gel $(2.2 \times 7)$ eluting with EtOAc/hexane (1:9) to EtOAc/hexane (3:7) in $10 \%$ increment rises $(50 \mathrm{~mL}$ each) to yield the title compound 20 (297 mg, $0.84 \mathrm{mmol}, 84 \%$ ) as a viscous colourless oil.

FT-IR $v_{\max }$ (neat) $1762 \mathrm{~s}, 1715 \mathrm{~s}, 1584 \mathrm{~m}, 1454 \mathrm{~m}, 1358 \mathrm{~m}, 1263 \mathrm{~s}, 1153 \mathrm{~s} \mathrm{~cm}^{-1}$.

${ }^{1}$ H NMR (400 MHz) $\delta$ 7.36-7.21 (6H, m, PhH), 6.89-6.83 (3H, m, PhH), $5.29(1 \mathrm{H}, \mathrm{d}, J=9.3 \mathrm{~Hz},-$ CHPh), $4.51(1 \mathrm{H}, \mathrm{d}, J=12.0 \mathrm{~Hz},-O C H H A r), 4.43(1 \mathrm{H}, \mathrm{d}, J=12.0 \mathrm{~Hz},-$ OCHHAr), $4.00(1 \mathrm{H}, \mathrm{d}, J=10.8 \mathrm{~Hz},-\mathrm{CHCO}-), 3.81(3 \mathrm{H}, \mathrm{s},-\mathrm{OCH}), 3.48$ $(1 \mathrm{H}, \mathrm{dd}, J=10.3,3.5 \mathrm{~Hz},-\mathrm{CHCHH}-), 3.46(1 \mathrm{H}, \mathrm{dd}, J=10.3,3.5 \mathrm{~Hz},-$ CHCHH-), $3.10\left(1 \mathrm{H}, \mathrm{ddt}, J=10.5,9.5,3.5 \mathrm{~Hz}-\mathrm{CHCH}_{2}-\right), 2.47(3 \mathrm{H}, \mathrm{s},-$ $\mathrm{COCH}_{3}$ ); keto/enol ratio 10:1 - enolic resonances were observed at $\delta 11.27$ $(1 \mathrm{H}, \mathrm{s}), 5.49(1 \mathrm{H}, \mathrm{d}, J=2.0 \mathrm{~Hz}), 4.56(1 \mathrm{H}, \mathrm{d}, J=12.8 \mathrm{~Hz}), 1.92(3 \mathrm{H}, \mathrm{s})$.

${ }^{13} \mathbf{C}$ NMR $(100 \mathrm{MHz}) \delta 200.8\left(\mathbf{C O}_{\mathrm{ket}}\right), 171.7\left(\mathbf{C O}_{\mathrm{est}}\right), 160.3\left(\mathbf{C}_{\mathrm{ar}}\right), 139.5\left(\mathbf{C}_{\mathrm{ar}}\right), 137.7\left(\mathbf{C}_{\mathrm{ar}}\right), 130.1$ $\left(\mathbf{C H}_{\mathrm{ar}}\right), 129.4\left(\mathbf{C H}_{\mathrm{ar}}\right), 129.2\left(\mathbf{C H}_{\mathrm{ar}}\right), 126.8\left(\mathbf{C H}_{\mathrm{ar}}\right), 120.5\left(\mathbf{C H}_{\mathrm{ar}}\right), 114.0$ $\left(\mathbf{C H}_{\mathrm{ar}}\right), 113.7\left(\mathbf{C H}_{\mathrm{ar}}\right), 81.4(-\mathbf{C H P h}), 73.5\left(-\mathrm{CH}_{2} \mathrm{Ar}\right), 65.7\left(-\mathrm{CHCH}_{2} \mathrm{O}-\right), 56.0$ $(-\mathbf{C H C O}-), \quad 55.7 \quad\left(-\mathrm{OCH}_{3}\right), \quad 46.7 \quad\left(-\mathrm{CHCH}_{2}-\right), 30.6 \quad\left(-\mathrm{COCH}_{3}\right)$; enolic resonances were observed at $\delta 128.6,125.4,120.2,113.9,113.4,82.9,72.1$, $46.9,19.5$.

LRMS (ES +ve) $\quad m / z$ (relative intensity) $377(60)[\mathrm{M}+\mathrm{Na}]^{+}, 731(100)[2 \mathrm{M}+\mathrm{Na}]^{+}$.

HRMS $(E S+v e) \quad$ Calcd for $\mathrm{C}_{42} \mathrm{H}_{44} \mathrm{O}_{10} \mathrm{Na}$ (dimer) 731.2827, found 731.2856. 


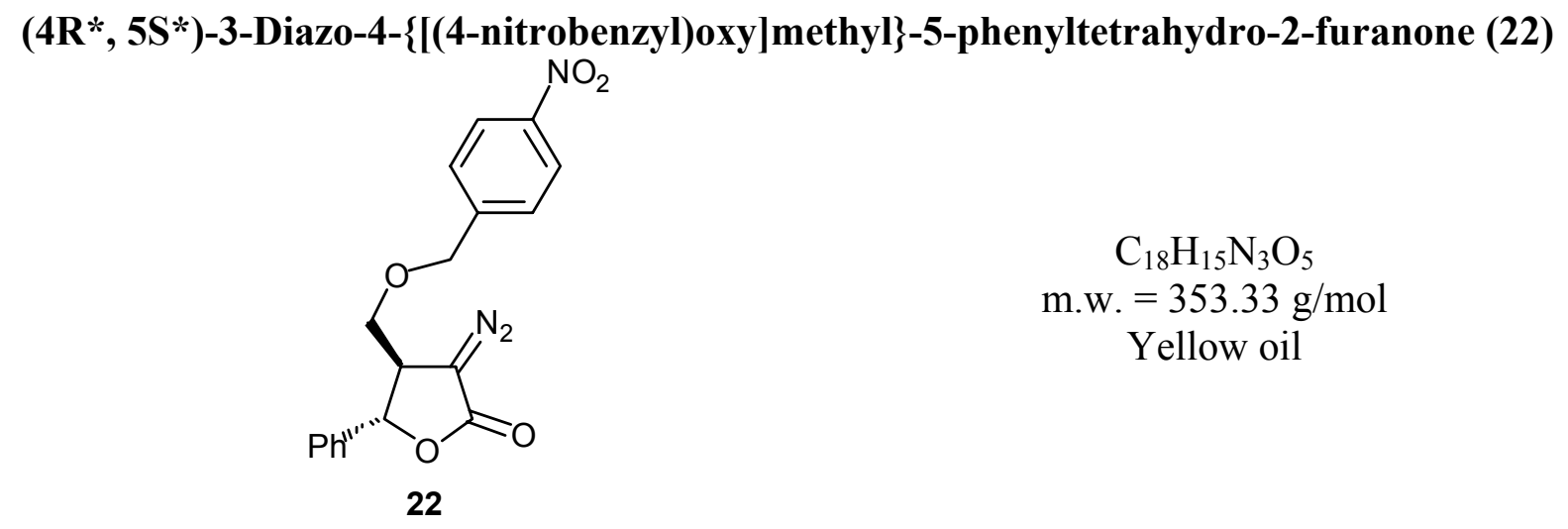

The title compound was prepared according to the method outlined for $\mathbf{2 3}$ whereby lactone $\mathbf{1 8}$ (92 $\mathrm{mg}, 0.25 \mathrm{mmol}), \mathrm{Tf}_{2} \mathrm{O}(168 \mu \mathrm{L}, 1.00 \mathrm{mmol})$ and $\mathrm{NaN}_{3}(130 \mathrm{mg}, 2.00 \mathrm{mmol})$ were reacted and worked up under the conditions described to yield a crude yellow foam (101 mg). Purification was accomplished by flash chromatography on silica gel $(2.2 \times 3.5)$ eluting with EtOAc/hexane (3:97) to EtOAc/hexane (1:4) in $3 \%$ increment rises $(20 \mathrm{~mL}$ each) to give the title compound $22(70 \mathrm{mg}$, $0.20 \mathrm{mmol}, 80 \%$ ) as a yellow oil.

FT-IR (neat) $v_{\max } \quad 2101 \mathrm{~s}, 1735 \mathrm{~s}, 1518 \mathrm{~s}, 1376 \mathrm{~m}, 1345 \mathrm{~s}, 1261 \mathrm{~m}, 1107 \mathrm{~s} \mathrm{~cm}^{-1}$.

${ }^{1}$ H NMR $(300 \mathrm{MHz}) \delta 8.24(2 \mathrm{H}, \mathrm{d}, J=8.8 \mathrm{~Hz}, \mathrm{PhH}), 7.47(2 \mathrm{H}, \mathrm{d}, J=8.8 \mathrm{~Hz}, \mathrm{PhH}), 7.44-7.32$ $(5 \mathrm{H}, \mathrm{m}, \mathrm{PhH}), 5.20$ (1H, d, $J=4.4 \mathrm{~Hz}, \mathrm{PhCH}-), 4.71\left(2 \mathrm{H}, \mathrm{s},-\mathrm{OCH}_{2} \mathrm{PNP}\right)$, 3.93-3.78 (3H, m, $-\mathrm{CH}_{2} \mathrm{OPNB}$ and $\left.-\mathrm{CHCH}_{2}-\right)$.

${ }^{13} \mathbf{C ~ N M R}(75 \mathrm{MHz}) \quad \delta 169.2(\mathbf{C O}), 147.8\left(\mathbf{C}_{\mathrm{ar}}\right), 144.7\left(\mathbf{C}_{\mathrm{ar}}\right), 138.8\left(\mathbf{C}_{\mathrm{ar}}\right), 129.2\left(\mathbf{C H}_{\mathrm{ar}}\right), 127.8$ $\left(\mathbf{C H}_{\mathrm{ar}}\right), 125.5\left(\mathbf{C H}_{\mathrm{ar}}\right), 124.0\left(\mathrm{CH}_{\mathrm{ar}}\right), 80.5(\mathrm{PhCH}-), 72.5\left(\mathrm{PNPCH}_{2} \mathrm{O}-\right), 71.8\left(-\mathbf{C H}_{2} \mathrm{OPNB}\right), 52.8(-$ $\left.\mathrm{CN}_{2}\right), 45.4\left(-\mathrm{CHCH}_{2}-\right)$. 


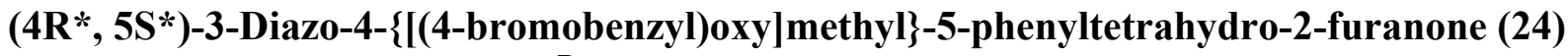

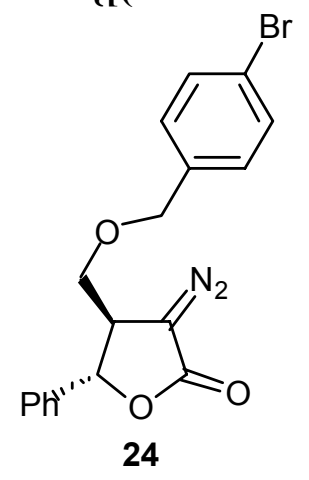

\author{
$\mathrm{C}_{18} \mathrm{H}_{15} \mathrm{~N}_{2} \mathrm{O}_{3} \mathrm{Br}$ \\ m.w. $=387.23 \mathrm{~g} / \mathrm{mol}$ \\ Yellow oil
}

The title compound was prepared according to the method outlined for $\mathbf{2 3}$, whereby reaction of lactone 19 (500 mg, $1.24 \mathrm{mmol})$ with $\mathrm{Tf}_{2} \mathrm{O}(0.83 \mathrm{~mL}, 4.96 \mathrm{mmol})$ and $\mathrm{NaN}_{3}(645 \mathrm{mg}, 9.92 \mathrm{mmol})$ and workup under the conditions described gave a crude yellow foam $(750 \mathrm{mg}$ ). Purification was accomplished by flash chromatography on silica gel $(2.8 \times 3.5)$ eluting with EtOAc/hexane (1:20) to EtOAc/hexane (1:4) in $5 \%$ increment rises $(50 \mathrm{~mL}$ each) to yield the title compound 24 (399 mg, $1.03 \mathrm{mmol}, 83 \%$ ) as a bright yellow oil.

FT-IR (neat) $v_{\max } \quad 2100 \mathrm{~s}, 1711 \mathrm{~s}, 1485 \mathrm{~m}, 1369 \mathrm{~s}, 1263 \mathrm{~s}, 1208 \mathrm{~s}, 1108,1007 \mathrm{~s} \mathrm{~cm}^{-1}$.

${ }^{1}$ H NMR (300 MHz) $\delta 7.50(2 \mathrm{H}, \mathrm{d}, J=8.5 \mathrm{~Hz}, \operatorname{ArH}), 7.45-7.23(5 \mathrm{H}, \mathrm{m}, \mathrm{PhH}), 7.18(2 \mathrm{H}, \mathrm{d}, J=$ $8.2 \mathrm{~Hz}, \mathrm{ArH}), 5.16(1 \mathrm{H}, \mathrm{d}, J=4.0 \mathrm{~Hz}, \mathrm{PhCH}-), 4.54\left(2 \mathrm{H}, \mathrm{s}, \quad-\mathrm{OC} \mathbf{H}_{2} \mathrm{Ar}\right)$, $3.80(1 \mathrm{H}, \mathrm{dd}, J=12.0,2.6 \mathrm{~Hz},-\mathrm{CHCHHO}-), 3.77-3.70$ (2H, m, -CHCHHOand $\left.-\mathrm{CHCH}_{2}-\right)$.

${ }^{13} \mathbf{C}$ NMR $(75 \mathrm{MHz}) \quad \delta 169.5(\mathbf{C O}), 139.2\left(\mathbf{C}_{\mathrm{ar}}\right), 136.6\left(\mathbf{C}_{\mathrm{ar}}\right), 132.2\left(\mathbf{C H}_{\mathrm{ar}}\right), 129.7\left(\mathbf{C H}_{\mathrm{ar}}\right), 129.4$ $\left(\mathrm{CH}_{\mathrm{ar}}\right), 125.8\left(\mathrm{CH}_{\mathrm{ar}}\right), 122.4\left(\mathbf{C}_{\mathrm{ar}}\right), 80.8(\mathrm{PhCH}-), 73.3\left(\mathrm{ArCH}_{2} \mathrm{O}-\right), 71.3(-$ $\left.\mathrm{CH}_{2} \mathrm{OBn}\right), 53.1\left(-\mathrm{CN}_{2}\right), 45.7\left(-\mathrm{CHCH}_{2}-\right)$. 


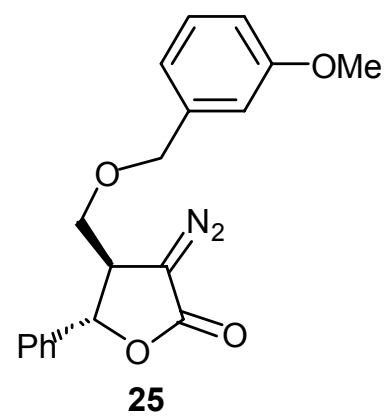

\author{
$\mathrm{C}_{19} \mathrm{H}_{18} \mathrm{~N}_{2} \mathrm{O}_{4}$ \\ m.w. $=338.36 \mathrm{~g} / \mathrm{mol}$ \\ Yellow oil
}

The title compound was prepared according to the method outlined for $\mathbf{2 3}$ (method B), whereby reaction of lactone 20 (248 $\mathrm{mg}, 0.70 \mathrm{mmol})$ with $\mathrm{Tf}_{2} \mathrm{O}(0.47 \mathrm{~mL}, 2.80 \mathrm{mmol})$ and $\mathrm{NaN}_{3}(364 \mathrm{mg}$, $5.60 \mathrm{mmol}$ ) and workup under the conditions described gave a crude yellow foam (573 $\mathrm{mg})$. Purification was accomplished by flash chromatography on silica gel $(2.2 \mathrm{x} 3)$ eluting with EtOAc/hexane $(1: 20)$ to EtOAc/hexane (1:4) in $5 \%$ increment rises (30 mL each) to yield the title compound 25 (198 mg, $0.59 \mathrm{mmol}, 84 \%$ ) as a bright yellow oil.

FT-IR (neat) $v_{\max } 2094 \mathrm{~s}, 1728 \mathrm{~s}, 1453 \mathrm{~m}, 1371 \mathrm{~m}, 1258 \mathrm{~s}, 1103 \mathrm{~s}, 1012 \mathrm{~s} \mathrm{~cm}^{-1}$.

${ }^{1}$ H NMR (300 MHz) $\delta$ 7.48-7.31 (6H, m, PhH), 6.95-6.90 (3H, m, PhH), $5.21(1 \mathrm{H}, \mathrm{d}, J=4.3 \mathrm{~Hz}$, $\mathrm{PhCH}-), 4.62$ (2H, s, -OCH $2 \mathrm{Ar}), 3.88\left(3 \mathrm{H}, \mathrm{s},-\mathrm{OCH}_{3}\right), 3.89-3.77(3 \mathrm{H}, \mathrm{m},-$ $\mathrm{CHCH}_{2-}$ and $-\mathrm{CHCH}_{2-}$ ).

${ }^{13} \mathbf{C}$ NMR $(75 \mathrm{MHz}) \quad \delta 169.6(\mathbf{C O}), 160.3\left(\mathbf{C}_{\mathrm{ar}}\right), 139.3\left(\mathbf{C}_{\mathrm{ar}}\right), 139.2\left(\mathbf{C}_{\mathrm{ar}}\right), 130.1\left(\mathbf{C H}_{\mathrm{ar}}\right), 129.4$ $\left(\mathbf{C H}_{\mathrm{ar}}\right), 129.3\left(\mathrm{CH}_{\mathrm{ar}}\right), 125.8\left(\mathrm{CH}_{\mathrm{ar}}\right), 120.3\left(\mathbf{C H}_{\mathrm{ar}}\right), 114.1\left(\mathbf{C H}_{\mathrm{ar}}\right), 113.4$ $\left(\mathrm{CH}_{\mathrm{ar}}\right), 80.8$ (PhCH-), $73.9\left(\mathrm{ArCH}_{2} \mathrm{O}-\right), 71.1$

$\left(-\mathrm{CH}_{2} \mathrm{OBn}\right), 55.7$ ($\left.\mathrm{OCH}_{3}\right), 53.2\left(-\mathrm{CN}_{2}\right), 45.8\left(-\mathrm{CHCH}_{2}-\right)$. 


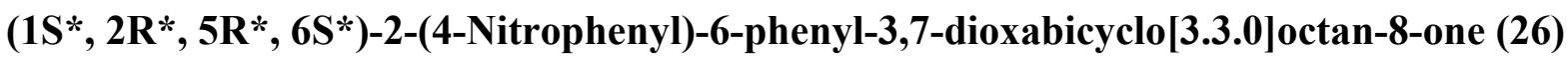<smiles>O=C1O[C@H](c2ccccc2)[C@@H]2COC(c3ccc([N+](=O)[O-])cc3)[C@H]12</smiles>

26

\author{
$\mathrm{C}_{18} \mathrm{H}_{15} \mathrm{NO}_{5}$ \\ m.w. $=325.32 \mathrm{~g} / \mathrm{mol}$ \\ Pale yellow solid
}

The title compound was prepared according to the method outlined for 27, whereby reaction of diazo lactone 22 (65 $\mathrm{mg}, 0.18 \mathrm{mmol}$ ) with dirhodium (II) tetraacetate (2 $\mathrm{mg}$, cat.) and workup under the conditions described gave crude furofuranone as a yellow foam (59 $\mathrm{mg})$. Triturating with $\mathrm{Et}_{2} \mathrm{O}$ gave the product as a very pale yellow powdery solid $(35 \mathrm{mg}, 0.11 \mathrm{mmol}, 60 \%)$. Purification of the filtrate was accomplished by flash chromatography on silica gel $(2 \times 6.5)$ eluting with $\mathrm{Et}_{2} \mathrm{O} /$ hexane (1:1 then $\left.4: 1\right)$ to yield the title compound $26(7 \mathrm{mg}, 0.02 \mathrm{mmol}, 12 \%)$ as a pale yellow solid - overall yield (42 $\mathrm{mg}, 0.13 \mathrm{mmol}, 72 \%)$.

MP $175-177{ }^{\circ} \mathrm{C}\left(\mathrm{Et}_{2} \mathrm{O}\right)$.

FT-IR (neat) $v_{\max } \quad 1762 \mathrm{~s}, 1512 \mathrm{~m}, 1347 \mathrm{~s}, 1172 \mathrm{~s}, 1072 \mathrm{~m}, 1019 \mathrm{~m} \mathrm{~cm}^{-1}$.

${ }^{1}$ H NMR (400 MHz) $\delta 8.25(2 \mathrm{H}, \mathrm{d}, J=8.5 \mathrm{~Hz}, \mathrm{PhH}), 7.59(2 \mathrm{H}, \mathrm{d}, J=9.0 \mathrm{~Hz}, \mathrm{PhH}), 7.44-7.35$ (3H, m, PhH), 7.34-7.30 (2H, m, PhH), $5.30(1 \mathrm{H}, \mathrm{d}, J=6.0 \mathrm{~Hz}, \mathrm{PhCH}-)$, $5.17(1 \mathrm{H}, \mathrm{d}, J=8.0 \mathrm{~Hz}$, PNPCH- $), 4.42(1 \mathrm{H}, \mathrm{d}, J=9.5 \mathrm{~Hz},-\mathrm{CHH}-), 4.03$ (1H, dd, $J=10.0,5.0 \mathrm{~Hz},-\mathrm{CHH}-), 3.68(1 \mathrm{H}, \mathrm{t}, J=8.5 \mathrm{~Hz},-\mathrm{COCH}-), 3.32$ (1H, ddd, $\left.J=10.5,9.5,5.5, \quad-\mathrm{CH}_{2} \mathrm{CH}-\right)$.

${ }^{13} \mathbf{C}$ NMR $(100 \mathrm{MHz}) \delta 174.1(\mathbf{C O}), 148.3\left(\mathbf{C}_{\mathrm{ar}}\right), 143.7\left(\mathbf{C}_{\mathrm{ar}}\right), 139.6\left(\mathbf{C}_{\mathrm{ar}}\right), 129.5\left(\mathbf{C H}_{\mathrm{ar}}\right), 129.4$ $\left(\mathbf{C H}_{\mathrm{ar}}\right), 127.6\left(\mathbf{C H}_{\mathrm{ar}}\right), 125.7\left(\mathbf{C H}_{\mathrm{ar}}\right), 124.1\left(\mathbf{C H}_{\mathrm{ar}}\right), 86.2(\mathrm{PhCH}-) 83.1$ (PNPCH-), 73.2 (- $\left.\mathrm{CH}_{2}-\right), 51.6$ (-COCH-), $51.1 \quad\left(-\mathrm{CH}_{2} \mathbf{C H}-\right)$.

LRMS (EI) $\mathrm{m} / z$ (relative intensity) $325(6)[\mathrm{M}]^{\bullet+}, 104(100)$.

CHN Anal. Calcd for $\mathrm{C}_{18} \mathrm{H}_{15} \mathrm{NO}_{5}$ : C, 66.46; H, 4.65; N, 4.30. Found: C, 66.17; H, 4.66; $\mathrm{N}, 4.27$. 


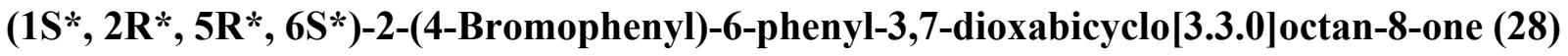<smiles>O=C1O[C@H](c2ccccc2)[C@@H]2CO[C@H](c3ccc(Br)cc3)[C@H]12</smiles>

28

\author{
$\mathrm{C}_{18} \mathrm{H}_{15} \mathrm{O}_{3} \mathrm{Br}$ \\ m.w. $=359.22 \mathrm{~g} / \mathrm{mol}$ \\ White crystalline solid
}

The title compound was prepared according to the method outlined for 27, whereby reaction of diazo lactone 24 (333 mg, $0.86 \mathrm{mmol}$ ) with dirhodium (II) tetraacetate (7 mg, cat.) and workup under the conditions described gave crude furofuranone as a pale yellow oil $(318 \mathrm{mg})$. Purification was accomplished by flash chromatography on silica gel $(3.2 \mathrm{x} 10)$ eluting with EtOAc/hexane (1:9) to EtOAc/heaxane $(1: 1)$ in $5 \%$ increment rises $(50 \mathrm{~mL}$ each) to yield the title compound 28 (234 mg, $0.65 \mathrm{mmol}, 76 \%$ ) as a white crystalline solid.

MP

FT-IR (neat) $v_{\max }$

${ }^{1} \mathbf{H}$ NMR (400 MHz) $135-137{ }^{\circ} \mathrm{C}($ EtOAc/hexane). (1761 s, $1490 \mathrm{w}, 1331 \mathrm{w}, 1255 \mathrm{~m}, 1168 \mathrm{~s}, 1061 \mathrm{~s}, 1010 \mathrm{~s} \mathrm{~cm}^{-1}$. $\delta 7.52(2 \mathrm{H}, \mathrm{d}, J=8.5 \mathrm{~Hz}, \mathrm{PhH}), 7.44-7.28(7 \mathrm{H}, \mathrm{m}, \mathrm{PhH}), 5.28(1 \mathrm{H}, \mathrm{d}, J=$ $6.5 \mathrm{~Hz}, \mathrm{PhCH}-), 5.05(1 \mathrm{H}, \mathrm{d}, J=8.5 \mathrm{~Hz}, \operatorname{ArCH}-), 4.36(1 \mathrm{H}, \mathrm{d}, J=9.5 \mathrm{~Hz},-$ CHH-), 3.95 (1H, dd, $J=9.5,4.8 \mathrm{~Hz},-\mathrm{CHH}-), 3.59(1 \mathrm{H}, \mathrm{t}, J=9.0 \mathrm{~Hz},-$ COCH-), 3.26 (1H, ddd, $\left.J=9.0,6.5,4.8 \quad-\mathrm{CH}_{2} \mathrm{CH}-\right)$.

${ }^{13} \mathbf{C}$ NMR $(100 \mathrm{MHz}) \delta 174.5(\mathbf{C O}), 139.9\left(\mathbf{C}_{\mathrm{ar}}\right), 135.5\left(\mathbf{C}_{\mathrm{ar}}\right), 132.1\left(\mathbf{C H}_{\mathrm{ar}}\right), 129.4\left(\mathbf{C H}_{\mathrm{ar}}\right), 129.3$ $\left(\mathbf{C H}_{\mathrm{ar}}\right), 128.4\left(\mathbf{C H}_{\mathrm{ar}}\right), 125.8\left(\mathrm{CH}_{\mathrm{ar}}\right), 122.8\left(\mathbf{C}_{\mathrm{ar}}\right), 86.0(\mathrm{PhCH}-), 83.6(\mathrm{ArCH}-$ ), $72.6\left(-\mathrm{CH}_{2}-\right), 51.7$ (-COCH-), $51.4\left(-\mathrm{CH}_{2} \mathbf{C H}-\right)$.

LRMS (ES +ve) $m / z$ (relative intensity) $741(100)[2 \mathrm{M}+\mathrm{Na}]^{+}$.

CHN Anal. Calcd for $\mathrm{C}_{18} \mathrm{H}_{15} \mathrm{O}_{3} \mathrm{Br}$ : C, 60.19; H, 4.21. Found: C, 60.29; H, 4.27. 


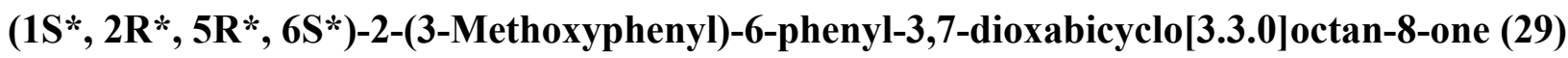<smiles>COc1cccc(C2OC[C@@H]3[C@@H]2C(=O)O[C@H]3c2ccccc2)c1</smiles>

29

\author{
$\mathrm{C}_{19} \mathrm{H}_{18} \mathrm{O}_{4}$ \\ m.w. $=310.35 \mathrm{~g} / \mathrm{mol}$ \\ White powdery solid
}

The title compound was prepared according to the method outlined for 27 , whereby reaction of diazo lactone 25 (160 mg, $0.47 \mathrm{mmol}$ ) with dirhodium (II) tetraacetate (4 mg, cat.) and workup under the conditions described gave crude furofuranone as a colourless oil (155 mg). Purification was accomplished by flash chromatography on silica gel $(2.2 \times 2.8)$ eluting with EtOAc/hexane (1:9) to EtOAc/heaxane (1:1) in $5 \%$ increment rises $(25 \mathrm{~mL}$ each) to yield the title compound 29 (118 $\mathrm{mg}, 0.38 \mathrm{mmol}, 82 \%$ ) as a white powdery solid.

MP

FT-IR (neat) $v_{\max }$

121-122 ${ }^{\circ} \mathrm{C}($ EtOAc/hexane).

${ }^{1}$ H NMR (400 MHz) $\delta$ 7.43-7.29 (6H, m, PhH), 7.01-6.96 (2H, m, PhH), $6.87(1 \mathrm{H}, \mathrm{ddd}, J=8.3$, 2.5, $0.8 \mathrm{~Hz}, \mathrm{PhH}), 5.30(1 \mathrm{H}, \mathrm{d}, J=6.5 \mathrm{~Hz}, \mathrm{PhCH}-), 5.07(1 \mathrm{H}, \mathrm{d}, J=8.5 \mathrm{~Hz}$, $\operatorname{ArCH}-), 4.36(1 \mathrm{H}, \mathrm{d}, J=9.8 \mathrm{~Hz},-\mathrm{CHH}-), 3.94(1 \mathrm{H}, \mathrm{dd}, J=9.8,4.8 \mathrm{~Hz}$, CHH-), 3.82 (3H, s, - $\left.\mathrm{OCH}_{3}\right), 3.58(1 \mathrm{H}, \mathrm{t}, J=8.8 \mathrm{~Hz},-\mathrm{COCH}-), 3.24(1 \mathrm{H}$, $\left.\mathrm{ddd}, J=9.0,6.5,4.8,-\mathrm{CH}_{2} \mathrm{CH}-\right)$.

${ }^{13} \mathbf{C}$ NMR $(100 \mathrm{MHz}) \delta 174.6(\mathbf{C O}), 160.2\left(\mathbf{C}_{\mathrm{ar}}\right), 140.0\left(\mathbf{C}_{\mathrm{ar}}\right), 138.1\left(\mathbf{C}_{\mathrm{ar}}\right), 130.0\left(\mathbf{C H}_{\mathrm{ar}}\right), 129.4$ $\left(\mathbf{C H}_{\mathrm{ar}}\right), 129.2\left(\mathbf{C H}_{\mathrm{ar}}\right), 125.8\left(\mathbf{C H}_{\mathrm{ar}}\right), 119.1\left(\mathbf{C H}_{\mathrm{ar}}\right), 114.2\left(\mathbf{C H}_{\mathrm{ar}}\right), 112.4$ $\left(\mathrm{CH}_{\mathrm{ar}}\right), 85.8$ (PhCH-) 84.2 (ArCH-), $72.3\left(-\mathrm{CH}_{2}-\right), 55.6\left(-\mathrm{OCH}_{3}\right), 51.9$ ($\mathrm{COCH}-), 51.6\left(-\mathrm{CH}_{2} \mathrm{CH}-\right)$.

LRMS $(\mathrm{ES}+\mathrm{ve}) \quad \mathrm{m} / z$ (relative intensity) $643(100)[2 \mathrm{M}+\mathrm{Na}]^{+}$.

CHN Anal.
Calcd for $\mathrm{C}_{19} \mathrm{H}_{18} \mathrm{O}_{4}$ : C, 73.53; H, 5.85. Found: C, 73.40; H, 5.92. 


\section{Rac-1-(3,4-Dimethoxy)phenyl-prop-2-en-1-ol (31706-95-3) ( $( \pm)-(30)$}<smiles>C=CC(O)c1ccc(OC)c(OC)c1</smiles>

$( \pm)-30$

\author{
$\mathrm{C}_{11} \mathrm{H}_{14} \mathrm{O}_{3}$ \\ m.w. $=194.23 \mathrm{~g} / \mathrm{mol}$ \\ Pale yellow solid
}

The title compound was prepared according to the method outlined for $( \pm)-\mathbf{3 1}$, whereby reaction of 3,4-dimethoxybenzaldehyde ( $49.85 \mathrm{~g}, 0.30 \mathrm{~mol})$ with vinyl magnesium bromide $(345 \mathrm{~mL}$ of a $1 \mathrm{M}$ sol in THF, $0.345 \mathrm{~mol}$ ) and work-up under the conditions described gave a crude yellow oil (68.2 g). Purification was accomplished by flash chromatography on silica gel $(8.5 \times 8)$ eluting with EtOAc/hexane (1:1) to yield the title compound $30(56.82,0.29 \mathrm{~mol}, 98 \%)$ as a very viscous yellow oil that crystallised on standing to provide a pale yellow, low-melting, solid. Spectroscopic details were consistent with those observed in the literature. ${ }^{1}$

MP $\quad 31-33^{\circ} \mathrm{C}$. Previously reported as an oil. ${ }^{1}$

FT-IR (neat) $v_{\max } \quad 3501 \mathrm{~s}, 1593 \mathrm{~m}, 1514 \mathrm{~s}, 1462 \mathrm{~s}, 1255 \mathrm{~s}, 1223 \mathrm{~s}, 1137 \mathrm{~s} \mathrm{~cm}^{-1}$.

${ }^{1}$ H NMR (400 MHz) $\delta 6.92-6.87(2 \mathrm{H}, \mathrm{m}, \mathrm{PhH}), 6.83(1 \mathrm{H}, \mathrm{d}, J=8.3 \mathrm{~Hz}, \mathrm{PhH}), 6.04(1 \mathrm{H}, \mathrm{ddd}, J=$ $\left.17.1,10.3,6.0 \mathrm{~Hz},-\mathrm{CH}=\mathrm{CH}_{2}\right), 5.34(1 \mathrm{H}, \mathrm{dt}, J=17.1,1.3 \mathrm{~Hz},-\mathrm{CH}=\mathrm{CHH})$, $5.19(1 \mathrm{H}, \mathrm{dt}, J=10.3,1.3 \mathrm{~Hz},-\mathrm{CH}=\mathrm{CHH}), 5.14(1 \mathrm{H}$, br d, $J=5.5 \mathrm{~Hz}$, $\mathrm{ArCH}-), 3.87\left(3 \mathrm{H}, \mathrm{s},-\mathrm{OCH}_{3}\right), 3.86\left(3 \mathrm{H}, \mathrm{s}, \quad-\mathrm{OCH}_{3}\right)$.

${ }^{13} \mathbf{C}$ NMR $(100 \mathrm{MHz}) \delta 149.5\left(\mathbf{C}_{\mathrm{ar}}\right), 149.0\left(\mathbf{C}_{\mathrm{ar}}\right), 140.7\left(-\mathbf{C H}=\mathrm{CH}_{2}\right), 135.7\left(\mathbf{C}_{\mathrm{ar}}\right), 119.1\left(\mathbf{C H}_{\mathrm{ar}}\right)$, $115.3\left(-\mathrm{CH}=\mathbf{C H}_{2}\right), 111.5\left(\mathbf{C H}_{\mathrm{ar}}\right), 110.0\left(\mathbf{C H}_{\mathrm{ar}}\right), 75.5 \quad$ (-CHAr), $56.4(-$ $\left.\mathrm{OCH}_{3}\right), 56.3\left(-\mathrm{OCH}_{3}\right)$.

LRMS $\left(\mathrm{CI}, \mathrm{NH}_{3}\right) \quad m / z$ (relative intensity) $176(100)\left[\mathrm{M}\left(-\mathrm{H}_{2} \mathrm{O}\right)\right]^{+}$.

\footnotetext{
${ }^{1}$ Ikeda, R.; Nagao, T.; Okabe, H.; Nakano, Y.; Matsunaga, H.; Katano, M.; Mori, M. Chem. Pharm. Bull. 1998, 46, $875-878$
} 
(4R, 5S)-5-(4-Methanesulfonyloxy-3-methoxy)phenyl-4-\{[(3,4-methylenedioxybenzyl) oxy]methyl\}-3-acetyl-tetrahydro-2-furanone (36a)

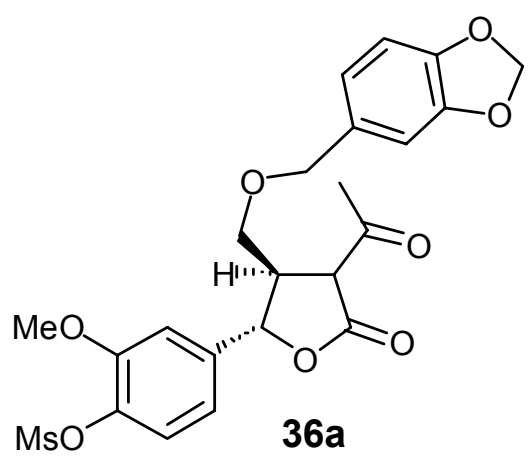

\author{
$\mathrm{C}_{23} \mathrm{H}_{24} \mathrm{SO}_{10}$ \\ m.w. $=492.50 \mathrm{~g} / \mathrm{mol}$
}

Viscous colourless oil

To a mixture of cyclopropane 35 (1.70 g, $5.0 \mathrm{mmol})$, 3,4-methylenedioxybenzyl alcohol (39a) (3.80 g, $25.0 \mathrm{mmol})$ and 2,6-di-tert-butylpyridine $(1.12 \mathrm{~mL}, 5.0 \mathrm{mmol})$ was added magnesium perchlorate $(223 \mathrm{mg}, 1.0 \mathrm{mmol})$ and the mixture heated to $120^{\circ} \mathrm{C}$ for $2 \mathrm{~h}$. The reaction mixture was allowed to cool to room temperature, diluted with EtOAc $(80 \mathrm{~mL})$ and treated with sat. $\mathrm{NH}_{4} \mathrm{Cl}(\mathrm{aq})$ $(80 \mathrm{~mL})$. The organic layer was separated and the aqueous extracted with EtOAc $(3 \times 80 \mathrm{~mL})$. The combined organic layers were washed with brine $(1 \times 80 \mathrm{~mL})$, dried with $\mathrm{Na}_{2} \mathrm{SO}_{4}$ and concentrated in vacuo to yield a crude yellow oil $(6.18 \mathrm{~g})$. Purification was accomplished by flash chromatography on silica gel ( 4 x 20) eluting with EtOAc/hexane (1:4 then 1:3, 1:2 and 1:1) to give the title compound $36 \mathrm{a}(1.33 \mathrm{~g}, 2.7 \mathrm{mmol}, 54 \%)$ as a viscous colourless oil, along with recovered cyclopropane $35(0.58 \mathrm{~g}, 1.7 \mathrm{mmol}, 34 \%)$ as an off-white powdery solid. Data for 36a.

$[\alpha]_{D}$ +33.6 (c. $0.67, \mathrm{CHCl}_{3}$ ).

FT-IR $v_{\max }$ (neat) $1768 \mathrm{~s}, 1711 \mathrm{~s}, 1503 \mathrm{~s}, 1359 \mathrm{~s}, 1240 \mathrm{~s}, 1152 \mathrm{~s}, 1114 \mathrm{~s}, 1032 \mathrm{~s} \mathrm{~cm}^{-1}$.

${ }^{1}$ H NMR (400 MHz) $\delta 7.27(1 \mathrm{H}, \mathrm{d}, J=8.3 \mathrm{~Hz}, \mathrm{PhH}), 6.92(1 \mathrm{H}, \mathrm{d}, J=1.8 \mathrm{~Hz}, \mathrm{PhH}), 6.81-6.73$ $(4 \mathrm{H}, \mathrm{m}, \mathrm{PhH}), 5.98\left(2 \mathrm{H} \mathrm{s},-\mathrm{OCH} \mathbf{H}_{2} \mathrm{O}\right), 5.27(1 \mathrm{H}, \mathrm{d}, J=9.3 \mathrm{~Hz},-\mathrm{CHAr}), 4.47$ (1H, d, $J=11.8 \mathrm{~Hz}$, -OCHHAr), 4.36 (1H, d, $J=11.8 \mathrm{~Hz}$, -OCHHAr), 3.99 $(1 \mathrm{H}, \mathrm{d}, J=10.5 \mathrm{~Hz},-\mathrm{CHCO}-), 3.87\left(3 \mathrm{H}, \mathrm{s},-\mathrm{OCH}_{3}\right), 3.48(1 \mathrm{H}, \mathrm{dd}, J=8.3$, $3.5 \mathrm{~Hz},-\mathrm{CHCHH}-), 3.45$ (1H, dd, $J=8.3,3.5 \mathrm{~Hz},-\mathrm{CHCHH}-), 3.19$ (3H, s, $\left.\mathrm{SO}_{2} \mathrm{CH}_{3}\right), 3.07\left(1 \mathrm{H}\right.$, ddt, $\left.J=10.5,9.3,3.5 \mathrm{~Hz}-\mathrm{CHCH}_{2}-\right), 2.50(3 \mathrm{H}, \mathrm{s},-$ $\mathrm{COCH}_{3}$ ); keto/enol ratio 10:1 - enolic resonances were observed at $\delta 11.24$ $(1 \mathrm{H}, \mathrm{s}), 5.48(1 \mathrm{H}, \mathrm{d}, J=2.5 \mathrm{~Hz}), 4.53(1 \mathrm{H}, \mathrm{d}, J=11.5 \mathrm{~Hz}), 1.97(3 \mathrm{H}, \mathrm{s})$.

${ }^{13} \mathbf{C}$ NMR $(100 \mathrm{MHz}) \delta 200.6\left(\mathbf{C O}_{\mathrm{ket}}\right), 171.4\left(\mathbf{C O}_{\mathrm{est}}\right), 152.3\left(\mathbf{C}_{\mathrm{ar}}\right), 148.4\left(\mathbf{C}_{\mathrm{ar}}\right), 148.0\left(\mathbf{C}_{\mathrm{ar}}\right), 139.0$ $\left(\mathbf{C}_{\mathrm{ar}}\right), 138.4\left(\mathbf{C}_{\mathrm{ar}}\right), 131.6\left(\mathbf{C}_{\mathrm{ar}}\right), 125.1\left(\mathbf{C H}_{\mathrm{ar}}\right), 122.1\left(\mathbf{C H}_{\mathrm{ar}}\right), 119.4\left(\mathbf{C H}_{\mathrm{ar}}\right)$, $110.8\left(\mathbf{C H}_{\mathrm{ar}}\right), 108.9\left(\mathbf{C H}_{\mathrm{ar}}\right), 108.7\left(\mathbf{C H}_{\mathrm{ar}}\right), 101.6 \quad\left(-\mathrm{OCH}_{2} \mathrm{O}-\right), 80.7(-$ CHAr), 73.6 (- $\left.\mathrm{CH}_{2} \mathrm{Ar}\right), 65.5\left(-\mathrm{CHCH}_{2} \mathrm{O}-\right), 56.5\left(-\mathrm{OCH}_{3}\right), 55.9$ (-CHCO-), $46.5\left(-\mathrm{CHCH}_{2}-\right), 38.8\left(-\mathrm{SO}_{2} \mathrm{CH}_{3}\right), 30.6\left(-\mathrm{COCH}_{3}\right)$; enolic resonances were observed at $\delta 125.2,121.8,117.6,108.8,108.6,82.2,73.7,71.8,46.9,19.6$.

LRMS $(\mathrm{ES}+\mathrm{ve}) \quad \mathrm{m} / \mathrm{z}$ (relative intensity) $515(90)[\mathrm{M}+\mathrm{Na}]^{+}, 1007(100)[2 \mathrm{M}+\mathrm{Na}]^{+}$. HRMS (ES +ve) Calcd for $\mathrm{C}_{23} \mathrm{H}_{24} \mathrm{SO}_{10} \mathrm{Na} 515.0982$, found 515.0985. 
$(4 \mathrm{R}$, 5S)-5-(4-Methanesulfonyloxy-3-methoxy)phenyl-3-diazo-4-\{[(3,4-methylenedioxybenzyl)oxy]methyl tetrahydro-2-furanone (37a)

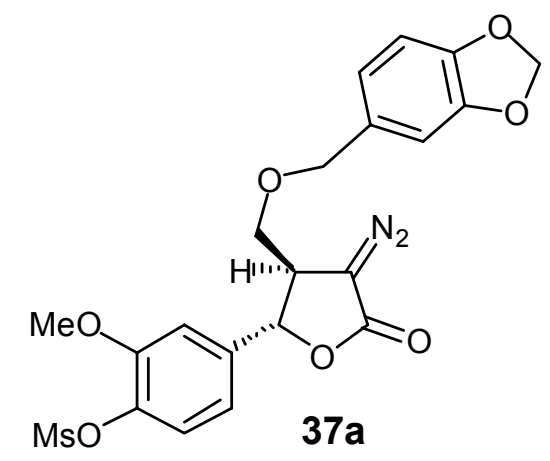

\author{
$\mathrm{C}_{21} \mathrm{H}_{20} \mathrm{~N}_{2} \mathrm{SO}_{9}$ \\ m.w. $=476.46 \mathrm{~g} / \mathrm{mol}$ \\ Bright yellow oil/foam
}

The title compound was prepared according to the method outlined for $\mathbf{2 3}$, whereby reaction of lactone 36a (1.00 g, $2.03 \mathrm{mmol})$ with $\mathrm{Tf}_{2} \mathrm{O}(1.37 \mathrm{~mL}, 8.12 \mathrm{mmol})$ and $\mathrm{NaN}_{3}(1.06 \mathrm{~g}, 16.24 \mathrm{mmol})$ and workup under the conditions described gave a crude yellow oil (1.12 g). Purification was accomplished by flash chromatography on silica gel $(4 \times 6)$ eluting with EtOAc/hexane (1:1) to give the title compound $\mathbf{3 7 a}(848 \mathrm{mg}, 1.78 \mathrm{mmol}, 88 \%$ ) as a viscous bright yellow oil/foam.

$[\alpha]_{D}$ +44.7 (c. $\left.0.28, \mathrm{CHCl}_{3}\right)$.

FT-IR $v_{\max }$ (neat) $\quad 2103 \mathrm{~s}, 1736 \mathrm{~s}, 1504 \mathrm{~m}, 1365 \mathrm{~s}, 1256 \mathrm{~m}, 1116 \mathrm{~m}, 1035 \mathrm{~m} \mathrm{~cm}^{-1}$.

${ }^{1}$ H NMR $(400 \mathrm{MHz}) \delta 7.29(1 \mathrm{H}, \mathrm{d}, J=8.3 \mathrm{~Hz}, \mathrm{PhH}), 6.96(1 \mathrm{H}, \mathrm{d}, J=2.0 \mathrm{~Hz}, \mathrm{PhH}), 6.87(1 \mathrm{H}, \mathrm{dd}$, $J=8.3,2.0 \mathrm{~Hz}, \mathrm{PhH}), 6.80-6.73(3 \mathrm{H}, \mathrm{m}, \mathrm{PhH}), 5.96\left(2 \mathrm{H} \mathrm{s},-\mathrm{OCH}_{2} \mathrm{O}-\right), 5.17$ $(1 \mathrm{H}, \mathrm{d}, J=4.8 \mathrm{~Hz},-\mathrm{CHAr}), 4.48\left(2 \mathrm{H}, \mathrm{s}, \quad-\mathrm{OCH}_{2} \mathrm{Ar}\right), 3.86\left(3 \mathrm{H}, \mathrm{s},-\mathrm{OCH}_{3}\right)$, 3.78-3.65 (3H, m, $-\mathrm{CHCH}_{2}$ - and $\left.-\mathrm{CHCH}_{2}-\right), 3.18\left(3 \mathrm{H}, \mathrm{s},-\mathrm{SO}_{2} \mathrm{CH}_{3}\right)$.

${ }^{13} \mathbf{C}$ NMR $(100 \mathrm{MHz}) \delta 169.3(\mathbf{C O}), 152.4\left(\mathbf{C}_{\mathrm{ar}}\right), 148.5\left(\mathbf{C}_{\mathrm{ar}}\right), 148.0\left(\mathbf{C}_{\mathrm{ar}}\right), 139.9\left(\mathbf{C}_{\mathrm{ar}}\right), 138.8\left(\mathbf{C}_{\mathrm{ar}}\right)$, $131.3\left(\mathbf{C}_{\mathrm{ar}}\right), 125.4\left(\mathbf{C H}_{\mathrm{ar}}\right), 122.0\left(\mathbf{C H}_{\mathrm{ar}}\right), 118.2\left(\mathbf{C H}_{\mathrm{ar}}\right), 110.2\left(\mathbf{C H}_{\mathrm{ar}}\right), 108.8$ $\left(\mathbf{C H}_{\mathrm{ar}}\right), 108.7\left(\mathbf{C H}_{\mathrm{ar}}\right), 101.6\left(-\mathrm{OCH}_{2} \mathrm{O}-\right), 80.2$ (-CHAr), $74.0\left(-\mathrm{CH}_{2} \mathrm{Ar}\right), 70.6$ $\left(-\mathrm{CHCH}_{2} \mathrm{O}-\right), 56.6\left(-\mathrm{OCH}_{3}\right), 52.9\left(\mathrm{CN}_{2}\right), 45.6\left(-\mathrm{CHCH}_{2}-\right), 38.9\left(-\mathrm{SO}_{2} \mathbf{C H}_{3}\right)$. 
(1S, 2R, 5R, 6S)-2-(3,4-Methylenedioxy)phenyl-6-(4-methanesulfonyloxy-3-methoxy) phenyl3,7-dioxabicyclo[3.3.0]octan-8-one (38a)<smiles>COc1ccc([C@@H]2OC(=O)[C@@H]3[C@@H](c4ccc5c(c4)OCO5)OC[C@H]32)cc1OC</smiles>

\author{
$\mathrm{C}_{21} \mathrm{H}_{20} \mathrm{SO}_{9}$ \\ m.w. $=448.45 \mathrm{~g} / \mathrm{mol}$ \\ White powdery solid
}

The title compound was prepared according to the method outlined for $\mathbf{2 7}$, whereby reaction of diazo lactone $37 \mathrm{a}(800 \mathrm{mg}, 1.62 \mathrm{mmol})$ with dirhodium (II) tetraacetate $(14 \mathrm{mg}, 0.03 \mathrm{mmol})$ and workup under the conditions described gave crude furofuranone as a white foam $(740 \mathrm{mg})$. Purification was accomplished by flash chromatography on silica gel $(4 \times 8)$ eluting with EtOAc/hexane (1:1 then 2:1) to give the title compound 38a (601 $\mathrm{mg}, 1.34 \mathrm{mmol}, 83 \%)$ as a white powdery solid:

\begin{tabular}{|c|c|}
\hline $\mathbf{P}$ & Ac/hexane). \\
\hline$[\alpha]_{D}$ & $+89.0\left(\right.$ c. $\left.0.46, \mathrm{CHCl}_{3}\right)$. \\
\hline T-IR (neat) $v_{\max }$ & $1771 \mathrm{~s}, 1605 \mathrm{w}, 1504 \mathrm{~m}, 1363 \mathrm{~s}, 1252 \mathrm{~m}, 1170 \mathrm{~s}, 1035 \mathrm{~s} \mathrm{~cm}^{-1}$. \\
\hline H NMR (400 MHz) & 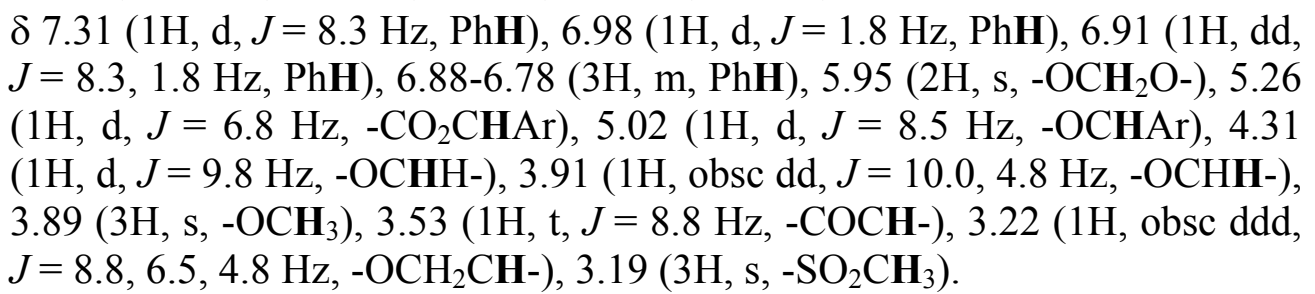 \\
\hline $0 \mathrm{MHz})$ & $\begin{array}{l}\delta 174.6(\mathbf{C O}), 152.4\left(\mathbf{C}_{\mathrm{ar}}\right), 148.4\left(\mathbf{C}_{\mathrm{ar}}\right), 148.2\left(\mathbf{C}_{\mathrm{ar}}\right), 140.4\left(\mathbf{C}_{\mathrm{ar}}\right), 138.8\left(\mathbf{C}_{\mathrm{ar}}\right), \\
130.2\left(\mathbf{C}_{\mathrm{ar}}\right), 125.4\left(\mathbf{C H}_{\mathrm{ar}}\right), 120.4\left(\mathbf{C H}_{\mathrm{ar}}\right), 118.2\left(\mathbf{C H}_{\mathrm{ar}}\right), 110.3\left(\mathrm{CH}_{\mathrm{ar}}\right), 108.8 \\
\left(\mathbf{C H}_{\mathrm{ar}}\right), 107.1\left(\mathbf{C H}_{\mathrm{ar}}\right), 101.6\left(-\mathbf{O C H}_{2} \mathrm{O}-\right), 85.0\left(-\mathrm{CO}_{2} \mathbf{C H A r}\right), 84.2(-\mathrm{CHAr}), \\
72.1\left(-\mathbf{C H}_{2}-\right), 56.7\left(-\mathrm{OCH}_{3}\right), 51.9 \quad(-\mathrm{COCH}-), 51.6\left(-\mathrm{CH}_{2} \mathbf{C H}-\right), 38.9(- \\
\left.\mathrm{SO}_{2} \mathbf{C H}_{3}\right) .\end{array}$ \\
\hline & \\
\hline & d: C, 55.98; H, 4.50 \\
\hline
\end{tabular}




\title{
(2R, 3R, 4S)-2[(3,4-Methylenedioxy)phenyl]-3-hydroxymethyl-4-\{[(4-methane-sulfonyloxy-3- methoxy)phenyl]hydroxy\}methyltetrahydrofuran (40a)
}

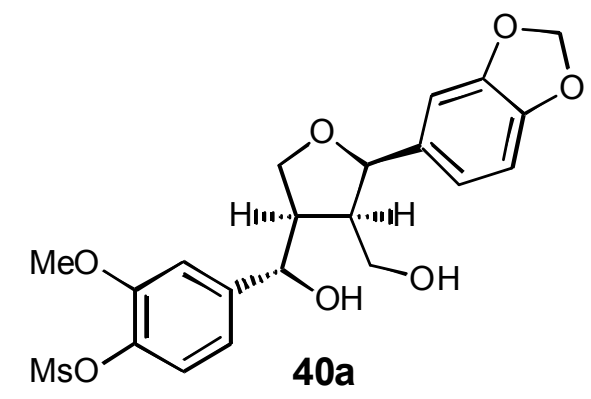

\author{
$\mathrm{C}_{21} \mathrm{H}_{24} \mathrm{SO}_{9}$ \\ m.w. $=452.48 \mathrm{~g} / \mathrm{mol}$ \\ White powdery solid
}

To a suspension of $\mathrm{LiAlH}_{4}(122 \mathrm{mg}, 3.21 \mathrm{mmol})$ in THF $(17 \mathrm{~mL})$ at $0{ }^{\circ} \mathrm{C}$ (ice bath) was added a solution of furofuranone 38a $(480 \mathrm{mg}, 1.07 \mathrm{mmol})$ in THF $(35 \mathrm{~mL})$ dropwise over $10 \mathrm{~min}$ and the grey suspension stirred for a further $10 \mathrm{~min}$. Water $(0.12 \mathrm{~mL}), 15 \% \mathrm{NaOH}(\mathrm{aq})(0.12 \mathrm{~mL})$ and water $(0.36 \mathrm{~mL})$ were sequentially added dropwise to the reaction mixture at $0{ }^{\circ} \mathrm{C}$, producing a pale grey/white granular precipitate that was filtered through Celite and washed with THF $(20 \mathrm{~mL})$ and EtOAc $(30 \mathrm{~mL})$. The filtrate was poured onto brine $(60 \mathrm{~mL})$, the organic phase separated and the aqueous extracted with EtOAc $(2 \times 60 \mathrm{~mL})$. The combined organic layers were washed with brine $(60 \mathrm{~mL})$, dried with $\mathrm{Na}_{2} \mathrm{SO}_{4}$ and concentrated in vacuo to yield a crude colourless oil (506 $\mathrm{mg}$ ). Purification was accomplished by flash chromatography on silica gel $(3 \times 9)$ eluting with EtOAc/hexane (1:1 then 2:1) to give the title compound $40 \mathrm{a}(359 \mathrm{mg}, 0.79 \mathrm{mmol}, 74 \%)$ as a white powdery solid along with lactol 41a $(63 \mathrm{mg}, 0.14 \mathrm{mmol}, 13 \%)$ as a white foam. Data for 40a.
MP
$158-160{ }^{\circ} \mathrm{C}$.
$[\alpha]_{D}$ +86.5 (c. $\left.0.13, \mathrm{CHCl}_{3}\right)$.
FT-IR (neat) $v_{\max } \quad 3375 \mathrm{br} \mathrm{w}, 1603 \mathrm{w}, 1502 \mathrm{~m}, 1490 \mathrm{~m}, 1361 \mathrm{~s}, 1112 \mathrm{~m}, 1033 \mathrm{~s} \mathrm{~cm}^{-1}$.
${ }^{1}$ H NMR $(400 \mathrm{MHz}) \delta 7.27(1 \mathrm{H}, \mathrm{d}, J=8.3 \mathrm{~Hz}, \mathrm{PhH}), 7.09(1 \mathrm{H}, \mathrm{d}, J=1.8 \mathrm{~Hz}, \mathrm{PhH}), 6.92(1 \mathrm{H}, \mathrm{dd}$, $J=8.3,1.8 \mathrm{~Hz}, \mathrm{PhH}), 6.80-6.71(3 \mathrm{H}, \mathrm{m}, \mathrm{PhH}), 5.95\left(2 \mathrm{H}, \mathrm{s},-\mathrm{OCH}_{2} \mathrm{O}-\right), 5.04$ $(1 \mathrm{H}, \mathrm{d}, J=5.3 \mathrm{~Hz},-\mathrm{OCHAr}), 4.80(1 \mathrm{H}, \mathrm{d}, J=10.3 \mathrm{~Hz}, \mathrm{ArCHOH}), 3.92(3 \mathrm{H}$, $\left.\mathrm{s},-\mathrm{OCH}_{3}\right), 3.74-3.58\left(3 \mathrm{H}, \mathrm{m}, \quad-\mathrm{OCH}_{2}\right.$ - and $\left.-\mathrm{CHHOH}\right), 3.39(1 \mathrm{H}, \mathrm{dd}, J=$ $10.8,1.8 \mathrm{~Hz},-\mathrm{CHHOH}), 3.18\left(3 \mathrm{H}, \mathrm{s},-\mathrm{SO}_{2} \mathrm{CH}_{3}\right), 3.04(1 \mathrm{H}, \mathrm{dq}, J=6.0,9.8$ $\left.\mathrm{Hz},-\mathrm{OCH}_{2} \mathbf{C H}-\right), 2.74-2.68\left(1 \mathrm{H}, \mathrm{m},-\mathrm{CHCH}_{2} \mathrm{OH}\right), 2.50(1 \mathrm{H}, \mathrm{br} \mathrm{s},-\mathrm{OH})$.
${ }^{13} \mathbf{C}$ NMR $(100 \mathrm{MHz}) \delta 152.2\left(\mathbf{C}_{\mathrm{ar}}\right), 148.3\left(\mathbf{C}_{\mathrm{ar}}\right), 147.3\left(\mathbf{C}_{\mathrm{ar}}\right), 143.7\left(\mathbf{C}_{\mathrm{ar}}\right), 138.3\left(\mathbf{C}_{\mathrm{ar}}\right), 133.1\left(\mathbf{C}_{\mathrm{ar}}\right)$, 125.0 $\left(\mathbf{C H}_{\mathrm{ar}}\right), 119.3\left(\mathrm{CH}_{\mathrm{ar}}\right), 119.2\left(\mathbf{C H}_{\mathrm{ar}}\right), 111.1\left(\mathbf{C H}_{\mathrm{ar}}\right), 108.7\left(\mathbf{C H}_{\mathrm{ar}}\right), 106.7$ $\left(\mathrm{CH}_{\mathrm{ar}}\right), 101.5\left(-\mathrm{OCH}_{2} \mathrm{O}-\right), 83.7$
$\left.\mathrm{OCH}_{2}-\right), 60.2\left(-\mathrm{CH}_{2} \mathrm{OH}\right), 56.6$ (-OCHAr), $73.6(-\mathrm{CHOH}), 69.0$ (- CHCHOH), $38.8\left(-\mathrm{SO}_{2} \mathrm{CH}_{3}\right)$.
LRMS (ES +ve) $\quad m / z$ (relative intensity) $927(100)[2 \mathrm{M}+\mathrm{Na}]^{+}$.
CHN Anal. Calcd for $\mathrm{C}_{21} \mathrm{H}_{24} \mathrm{SO}_{9}$ : C, 55.74; H, 5.35. Found: C, 55.77; H, 5.38. 
Data for (1S, 2R, 5R, 6S)-2-(3,4-Methylenedioxy)phenyl-6-(4-methanesulfonyloxy-3-methoxy) phenyl-3,7-dioxabicyclo[3.3.0]octan-8-ol (41a)<smiles>COc1ccc([C@H]2OC(O)[C@H]3[C@H]2CO[C@H]3c2ccc3c(c2)OCO3)cc1OC</smiles>

$$
\begin{gathered}
\mathrm{C}_{21} \mathrm{H}_{22} \mathrm{SO}_{9} \\
\text { m.w. }=450.46 \mathrm{~g} / \mathrm{mol} \\
\text { White foam }
\end{gathered}
$$

$[\alpha]_{\mathbf{D}}+84.2\left(\right.$ c. $\left.0.38, \mathrm{CHCl}_{3}\right)$.

FT-IR (neat) $v_{\max } \quad 3448 \mathrm{br}$ w, $1502 \mathrm{~m}, 1362 \mathrm{~s}, 1114 \mathrm{~m} \mathrm{~cm}^{-1}$.

${ }^{1}$ H NMR (400 MHz) $\delta 7.27(1 \mathrm{H}, \mathrm{d}, J=8.3 \mathrm{~Hz}, \mathrm{PhH}), 7.19(1 \mathrm{H}, \mathrm{d}, J=1.8 \mathrm{~Hz}, \mathrm{PhH}), 7.00(1 \mathrm{H}, \mathrm{dd}$, $J=8.3,1.8 \mathrm{~Hz}, \mathrm{PhH}), 6.94-6.79$ (3H, m, PhH), $5.97\left(2 \mathrm{H}, \mathrm{s},-\mathrm{OCH}_{2} \mathrm{O}-\right), 4.96$ $(1 \mathrm{H}$, br s, -OCHOH $), 4.91(1 \mathrm{H}, \mathrm{d}, J=6.8 \mathrm{~Hz},-\mathrm{CHAr}), 4.83(1 \mathrm{H}, \mathrm{d}, J=4.8$ $\mathrm{Hz}, \operatorname{ArCH}-), 4.14(1 \mathrm{H}, \mathrm{d}, J=9.5 \mathrm{~Hz},-\mathrm{OCHH}-), 3.90\left(3 \mathrm{H}, \mathrm{s},-\mathrm{OCH}_{3}\right), 3.85$ $(1 \mathrm{H}, \mathrm{dd}, J=9.3,4.5 \mathrm{~Hz},-\mathrm{OCHH}-), 3.18\left(3 \mathrm{H}, \mathrm{s},-\mathrm{SO}_{2} \mathrm{CH}_{3}\right), 3.15-3.08(2 \mathrm{H}$, m, -CHCHOH and - $\left.\mathrm{OCH}_{2} \mathrm{CH}-\right), 2.74$ (1H, br s, -OH).

${ }^{13} \mathbf{C}$ NMR $(100 \mathrm{MHz}) \delta 152.0\left(\mathbf{C}_{\mathrm{ar}}\right), 148.3\left(\mathbf{C}_{\mathrm{ar}}\right), 147.5\left(\mathbf{C}_{\mathrm{ar}}\right), 143.4\left(\mathbf{C}_{\mathrm{ar}}\right), 138.2\left(\mathbf{C}_{\mathrm{ar}}\right), 132.2\left(\mathbf{C}_{\mathrm{ar}}\right)$, $124.9\left(\mathbf{C H}_{\mathrm{ar}}\right), 120.0\left(\mathbf{C H}_{\mathrm{ar}}\right), 119.2\left(\mathbf{C H}_{\mathrm{ar}}\right), 111.4\left(\mathbf{C H}_{\mathrm{ar}}\right), 108.7\left(\mathbf{C H}_{\mathrm{ar}}\right), 107.4$ $\left(\mathbf{C H}_{\mathrm{ar}}\right), 101.6(-\mathbf{C H O H}), 101.6$ $\left(-\mathrm{OCH}_{2} \mathrm{O}-\right), 87.7$ (-CHAr), 82.5 (CHAr $), 72.1\left(-\mathrm{OCH}_{2}-\right), 57.6$ $(-\mathrm{CHCHOH}), 56.6\left(-\mathrm{OCH}_{3}\right), 53.5(-$ $\left.\mathrm{CHCH}_{2}-\right), 38.8\left(-\mathrm{SO}_{2} \mathbf{C H}_{3}\right)$.

LRMS $(\mathrm{ES}+\mathrm{ve}) \quad \mathrm{m} / z$ (relative intensity) $923(100)[2 \mathrm{M}+\mathrm{Na}]^{+}$.

HRMS (ES +ve) Calcd for $\mathrm{C}_{42} \mathrm{H}_{44} \mathrm{~S}_{2} \mathrm{O}_{18} \mathrm{Na}$ (dimer) 923.1861, found 923.1862. 


\title{
(1R, 2R, 5R, 6S)-2-(3,4-Methylenedioxy)phenyl-6-(4-methanesulfonyloxy-3-methoxy) phenyl-
} 3,7-dioxabicyclo[3.3.0]octane (42a)

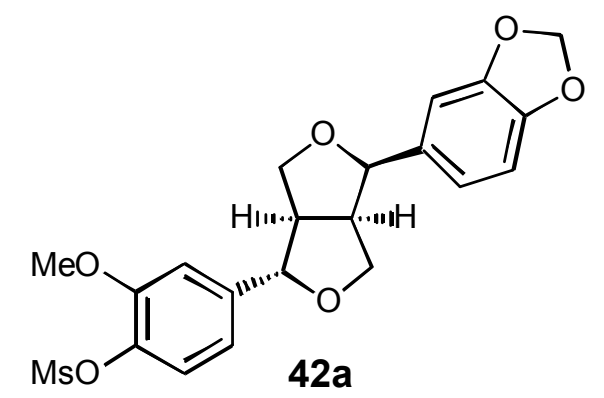

\author{
$\mathrm{C}_{21} \mathrm{H}_{22} \mathrm{SO}_{8}$ \\ m.w. $=434.47 \mathrm{~g} / \mathrm{mol}$ \\ White powdery solid
}

To a solution of diol 40a (320 mg, $0.71 \mathrm{mmol})$ in $\mathrm{CH}_{2} \mathrm{Cl}_{2}(25 \mathrm{~mL})$ at $0{ }^{\circ} \mathrm{C}$ (ice/salt bath) was added $\mathrm{NEt}_{3}(0.12 \mathrm{~mL}, 0.85 \mathrm{mmol})$ followed by DMAP $(2 \mathrm{mg}$, cat.) and the reaction stirred for $5 \mathrm{~min}$. A solution of $\mathrm{Ms}_{2} \mathrm{O}(185 \mathrm{mg}, 1.06 \mathrm{mmol})$ in $\mathrm{CH}_{2} \mathrm{Cl}_{2}(5 \mathrm{~mL})$ was added dropwise and the reaction allowed to warm to room temperature and stirred for $8 \mathrm{~h}$ before additional $\mathrm{Et}_{3} \mathrm{~N}(0.08 \mathrm{~mL}, 0.56$ mmol) and $\mathrm{Ms}_{2} \mathrm{O}$ (123 mg, $0.71 \mathrm{mmol}$ ) was added and stirred for a further $12 \mathrm{~h}$. TLC analysis still showed starting diol 39a so DMAP (7 mg, cat.) and $\mathrm{Ms}_{2} \mathrm{O}(123 \mathrm{mg}, 0.71 \mathrm{mmol})$ were again added and the mixture stirred for $20 \mathrm{~h}$. The reaction was then cooled to $0^{\circ} \mathrm{C}$ (ice bath), diluted with $\mathrm{CH}_{2} \mathrm{Cl}_{2}(10 \mathrm{~mL})$ and treated with $1 \mathrm{~N} \mathrm{HCl}(\mathrm{aq})(40 \mathrm{~mL})$. The mixture was allowed to warm to room temperature, the organic phase separated and the aqueous extracted with $\mathrm{CH}_{2} \mathrm{Cl}_{2}(3 \times 40 \mathrm{~mL})$. The combined organic layers were washed with brine $(40 \mathrm{~mL})$, dried with $\mathrm{Na}_{2} \mathrm{SO}_{4}$ and concentrated in vacuo to yield a pale orange oil $(475 \mathrm{mg})$. Purification was accomplished by flash chromatography on silica gel $(3.2 \times 6)$ eluting with EtOAc/hexane (1:3 then 1:2) to give the title compound 42a (233 $\mathrm{mg}, 0.54 \mathrm{mmol}, 76 \%)$ as a white powdery solid.
MP
$130-131^{\circ} \mathrm{C}$ (EtOAc/hexane).
$[\alpha]_{D}$ +125.2 (c. $0.38, \mathrm{CHCl}_{3}$ ).
FT-IR (neat) $v_{\max }$ $2870 \mathrm{w}, 1599 \mathrm{~m}, 1501 \mathrm{~m}, 1369 \mathrm{~s}, 1238 \mathrm{~s}, 1111 \mathrm{~s}, 1026 \mathrm{~s} \mathrm{~cm}^{-1}$.
${ }^{1}$ H NMR $(400 \mathrm{MHz}) \delta 7.27(1 \mathrm{H}, \mathrm{d}, J=8.3 \mathrm{~Hz}, \mathrm{PhH}), 7.04(1 \mathrm{H}, \mathrm{d}, J=1.5 \mathrm{~Hz}, \mathrm{PhH}), 6.92(1 \mathrm{H}, \mathrm{dd}$, $J=8.3,1.8 \mathrm{~Hz}, \mathrm{PhH}), 6.87(1 \mathrm{H}, \mathrm{s}, \mathrm{PhH}), 6.84-6.77(2 \mathrm{H}, \mathrm{m}, \mathrm{PhH}), 5.96(2 \mathrm{H}$, s, $\left.-\mathrm{OCH}_{2} \mathrm{O}-\right), 4.84\left(1 \mathrm{H}, \mathrm{d}, J=5.3 \mathrm{~Hz}, \quad-\mathrm{CHAr}^{1}\right), 4.48(1 \mathrm{H}, \mathrm{d}, J=7.0 \mathrm{~Hz}$, $\left.-\mathrm{CHAr}{ }^{2}\right), 4.14\left(1 \mathrm{H}, \mathrm{d}, J=9.5 \mathrm{~Hz}, \operatorname{Ar}^{1} \mathrm{CHOCHH}-\right), 3.90\left(3 \mathrm{H}, \mathrm{s},-\mathrm{OCH}_{3}\right)$, 3.90-3.82 (2H, m, $\mathrm{Ar}^{1} \mathrm{CHOCHH}-$ and $\left.\mathrm{Ar}^{2} \mathrm{CHOCHH}-\right), 3.37-3.26(2 \mathrm{H}, \mathrm{m}$, $\mathrm{Ar}^{2} \mathrm{CHOCHH}-$ and $\left.\mathrm{Ar}^{1} \mathrm{CHCH}-\right), 3.17\left(3 \mathrm{H}, \mathrm{s},-\mathrm{SO}_{2} \mathrm{CH}_{3}\right), 2.91-2.85(1 \mathrm{H}, \mathrm{m}$, $\left.\mathrm{Ar}^{2} \mathrm{CHCH}-\right)$.
${ }^{13} \mathbf{C}$ NMR $(100 \mathrm{MHz}) \delta 152.1\left(\mathbf{C}_{\mathrm{ar}}\right), 148.2\left(\mathbf{C}_{\mathrm{ar}}\right), 147.1\left(\mathbf{C}_{\mathrm{ar}}\right), 142.4\left(\mathbf{C}_{\mathrm{ar}}\right), 138.2\left(\mathbf{C}_{\mathrm{ar}}\right), 132.6\left(\mathbf{C}_{\mathrm{ar}}\right)$, $125.0\left(\mathbf{C H}_{\mathrm{ar}}\right), 119.2\left(\mathbf{C H}_{\mathrm{ar}}\right), 118.9\left(\mathbf{C H}_{\mathrm{ar}}\right), 110.8\left(\mathbf{C H}_{\mathrm{ar}}\right), 108.7\left(\mathbf{C H}_{\mathrm{ar}}\right), 106.9$ $\left(\mathbf{C H}_{\mathrm{ar}}\right), \quad 101.4 \quad\left(-\mathrm{OCH}_{2} \mathrm{O}-\right), \quad 87.5 \quad\left(\mathrm{Ar}{ }^{2} \mathbf{C H}-\right), \quad 82.5 \quad\left(\mathrm{Ar}^{1} \mathbf{C H}-\right), \quad 71.4$ $\left(\mathrm{Ar}^{1} \mathrm{CHOCH}_{2}-\right), 70.3\left(\mathrm{Ar}^{2} \mathrm{CHOCH}_{2}-\right), 56.6\left(-\mathrm{OCH}_{3}\right), 55.2\left(\mathrm{Ar}^{2} \mathrm{CHCH}-\right)$, $50.6\left(\mathrm{Ar}^{1} \mathrm{CHCH}-\right), 38.7\left(-\mathrm{SO}_{2} \mathrm{CH}_{3}\right)$.
LRMS (ES +ve) Where $\mathrm{Ar}^{1}=3,4$-methylenedioxy-, $\mathrm{Ar}^{2}=4$-methanesulfonyloxy-3-methoxy-
CHN Anal. $\mathrm{m} / z$ (relative intensity) $891(100)[2 \mathrm{M}+\mathrm{Na}]^{+}$.
Calcd for $\mathrm{C}_{21} \mathrm{H}_{22} \mathrm{SO}_{8}$ : C, 58.06; H, 5.10. Found: C, 58.17; H, 5.14. 
(1R, 2R， 5R， 6S)-2-(3,4-Methylenedioxy)phenyl-6-(4-hydroxy-3-methoxy)phenyl-3,7dioxabicyclo[3.3.0]octane. (+)-Xanthoxylol (1)<smiles>COc1cc([C@@H]2OC[C@H]3[C@H]2CO[C@H]3c2ccc3c(c2)OCO3)ccc1O</smiles>

$(+)-X a n t h o x y l o l(1)$

\author{
$\mathrm{C}_{20} \mathrm{H}_{20} \mathrm{O}_{6}$ \\ m.w. $=356.37 \mathrm{~g} / \mathrm{mol}$ \\ White powdery solid
}

The title compound was prepared according to a modified procedure described by Kawada et al. ${ }^{2}$ Thus, to a solution of furofuran $\mathbf{4 2 a}(130 \mathrm{mg}, 0.30 \mathrm{mmol})$ in dioxane/methanol $(8 \mathrm{~mL}, 1: 1)$ was added $3 \mathrm{~N} \mathrm{KOH} \mathrm{(aq)}(8 \mathrm{~mL})$. The cloudy mixture was stirred at $50{ }^{\circ} \mathrm{C}$ for $40 \mathrm{~h}$ and the resulting clear yellow solution diluted with EtOAc $(30 \mathrm{~mL})$ and acidified with $2 \mathrm{~N} \mathrm{HCl}(\mathrm{aq})(30 \mathrm{~mL})$. The organic layer was separated, the aqueous extracted with EtOAc $(3 \times 30 \mathrm{~mL})$ and the combined extracts were washed with brine $(30 \mathrm{~mL})$, dried with $\mathrm{Na}_{2} \mathrm{SO}_{4}$ and concentrated in vacuo to yield an off-white solid (113 mg). Purification was accomplished by flash chromatography on silica gel (2.2 $\times$ 8) eluting with EtOAc/hexane (1:1) to give the title compound 1 (96 mg, $0.27 \mathrm{mmol}, 90 \%$ ) as a white powdery solid. Spectroscopic details were consistent with those previously reported. ${ }^{3}$

MP 136-138 ${ }^{\circ} \mathrm{C}$ (lit. ${ }^{3 \mathrm{~b}} 138-140{ }^{\circ} \mathrm{C}$, lit. $\left.^{3 \mathrm{a}, 3 \mathrm{c}} 140-142{ }^{\circ} \mathrm{C}\right)$.

$[\alpha]_{\text {D }}+126.1\left(\right.$ c. $\left.0.38, \mathrm{CHCl}_{3}\right)\left(\right.$ lit. $^{3 \mathrm{~b}}+126-\mathrm{CHCl}_{3}$, lit. $\left.^{3 \mathrm{c}}+122-\mathrm{CHCl}_{3}\right)$.

FT-IR (neat) $v_{\max } \quad 3433$ br w, $1603 \mathrm{w}, 1492 \mathrm{~m}, 1438 \mathrm{~m}, 1226 \mathrm{~m}, 1073 \mathrm{~m}, 1029 \mathrm{~s} \mathrm{~cm}^{-1}$.

${ }^{1} \mathbf{H}$ NMR $(400 \mathrm{MHz}) \delta$ 6.91-6.78 (6H, m, PhH), $5.96\left(2 \mathrm{H}, \mathrm{s},-\mathrm{OCH} \mathrm{O}_{2}\right), 5.62(1 \mathrm{H}, \mathrm{s}, \quad-\mathrm{OH})$, $4.83\left(1 \mathrm{H}, \mathrm{d}, J=5.5 \mathrm{~Hz},-\mathbf{C H A r}^{1}\right), 4.41\left(1 \mathrm{H}, \mathrm{d}, J=7.0 \mathrm{~Hz}, \quad-\mathbf{C H A r}^{2}\right), 4.11$ $\left(1 \mathrm{H}, \mathrm{d}, J=9.5 \mathrm{~Hz}, \mathrm{Ar}^{1} \mathrm{CHOCHH}-\right), 3.90\left(3 \mathrm{H}, \mathrm{s}, \quad-\mathrm{OCH}_{3}\right), 3.89-3.80(2 \mathrm{H}$, $\mathrm{m}, \mathrm{Ar}^{1} \mathrm{CHOCHH}-$ and $\left.\mathrm{Ar}^{2} \mathrm{CHOCHH}-\right), 3.36-3.26$ (2H, m, $\mathrm{Ar}^{2} \mathrm{CHOCHH}-$ and $\left.\mathrm{Ar}^{1} \mathrm{CHCH}-\right), 2.93-2.85$ (1H, m, $\left.\mathrm{Ar}^{2} \mathrm{CHCH}-\right)$.

${ }^{13}$ C NMR $(100 \mathrm{MHz}) \delta 148.1\left(\mathbf{C}_{\mathrm{ar}}\right), 147.2\left(\mathbf{C}_{\mathrm{ar}}\right), 147.0\left(\mathbf{C}_{\mathrm{ar}}\right), 145.8\left(\mathbf{C}_{\mathrm{ar}}\right), 133.5\left(\mathbf{C}_{\mathrm{ar}}\right), 132.8\left(\mathbf{C}_{\mathrm{ar}}\right)$, $119.7\left(\mathbf{C H}_{\mathrm{ar}}\right), 119.2\left(\mathbf{C H}_{\mathrm{ar}}\right), 114.7\left(\mathbf{C H}_{\mathrm{ar}}\right), 109.1\left(\mathbf{C H}_{\mathrm{ar}}\right), 108.6\left(\mathbf{C H}_{\mathrm{ar}}\right), 106.9$

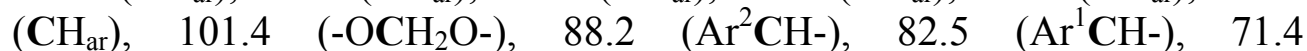
$\left(\mathrm{Ar}^{1} \mathrm{CHOCH}_{2}^{-}\right), 70.1\left(\mathrm{Ar}^{2} \mathrm{CHOCH}_{2}-\right), 56.4\left(-\mathrm{OCH}_{3}\right), 55.0\left(\mathrm{Ar}^{2} \mathrm{CHCH}-\right)$, $50.6\left(\mathrm{Ar}^{1} \mathrm{CHCH}-\right)$.

Where $\mathrm{Ar}^{1}=3,4$-methylenedioxy-, $\mathrm{Ar}^{2}=4$-hydroxy-3-methoxy-.

LRMS $(\mathrm{ES}+\mathrm{ve}) \quad \mathrm{m} / z$ (relative intensity) $713(100)[2 \mathrm{M}+\mathrm{H}]^{+}, 735(60)[2 \mathrm{M}+\mathrm{Na}]^{+}$.

CHN Anal. Calcd for $\mathrm{C}_{20} \mathrm{H}_{20} \mathrm{O}_{6}$ : C, 67.41; H, 5.66. Found: $\mathrm{C}$, 67.47; H, 5.65.

\footnotetext{
2 Yonezawa, S.; Komurasaki, T.; Kawada, K.; Tsuri, T.; Fuji, M.; Kugimiya, A.; Haga, N.; Mitsumori, S.; Inagaki, M.; Nakatani, T.; Tamura, Y.; Takechi, S.; Taishi, T.; Ohtani, M. J. Org. Chem. 1998, 63, 5831-5837.

3 (a) Abe, F.; Yahara, S.; Kubo, K.; Nonaka, G.; Okabe, H.; Nishioka, I. Chem. Pharm. Bull. 1974, 22, 2650-2655. (b) Ferreira, A. G.; Motidome, M.; Gottlieb, O. R.; Fernandes, J. B.; Vieira, P. C.; Cojocaru, M.; Gottlieb, H. E. Phytochemistry 1989, 28, 579-583. (c) Fang, J.-M.; Lee, C.-K.; Cheng, Y.-S. Phytochemistry 1992, $31,3659-3661$.
} 


\section{(1R, 2R, 5R, 6S)-2-(3,4-Methylenedioxy)phenyl-6-(3,4-dimethoxy)phenyl-3,7-} dioxabicyclo[3.3.0]octane. (+)-Methyl xanthoxylol (2)<smiles>COc1ccc([C@@H]2OC[C@H]3[C@H]2CO[C@H]3c2ccc3c(c2)OCO3)cc1OC</smiles>

(+)-Methylxanthoxylol (2)

\author{
$\mathrm{C}_{21} \mathrm{H}_{22} \mathrm{O}_{6}$ \\ m.w. $=370.40 \mathrm{~g} / \mathrm{mol}$ \\ White powdery solid
}

To a solution of furofuran $1(50 \mathrm{mg}, 0.14 \mathrm{mmol})$ in AnalaR acetone $(10 \mathrm{~mL})$ was added cesium carbonate $(69 \mathrm{mg}, 2.10 \mathrm{mmol})$ followed by methyl iodide $(87 \mu \mathrm{L}, 1.40 \mathrm{mmol})$ in one portion. The cloudy mixture was stirred at reflux for $18 \mathrm{~h}$ and the resulting clear colourless solution diluted with EtOAc $(20 \mathrm{~mL})$ and $1 \mathrm{~N} \mathrm{HCl}(\mathrm{aq})(15 \mathrm{~mL})$. The organic layer, separated and the aqueous extracted with EtOAc $(3 \times 20 \mathrm{~mL})$ and the combined extracts washed with brine $(20 \mathrm{~mL})$, dried with $\mathrm{Na}_{2} \mathrm{SO}_{4}$ and concentrated in vacuo to yield an off-white solid $(67 \mathrm{mg})$. Purification was accomplished by flash chromatography on silica gel $(2.2 \times 5)$ eluting with EtOAc/hexane (1:1) to give the title compound 2 (49 mg, $0.13 \mathrm{mmol}, 94 \%$ ) as a white powdery solid. Spectroscopic details were consistent with those previously reported. ${ }^{4}$

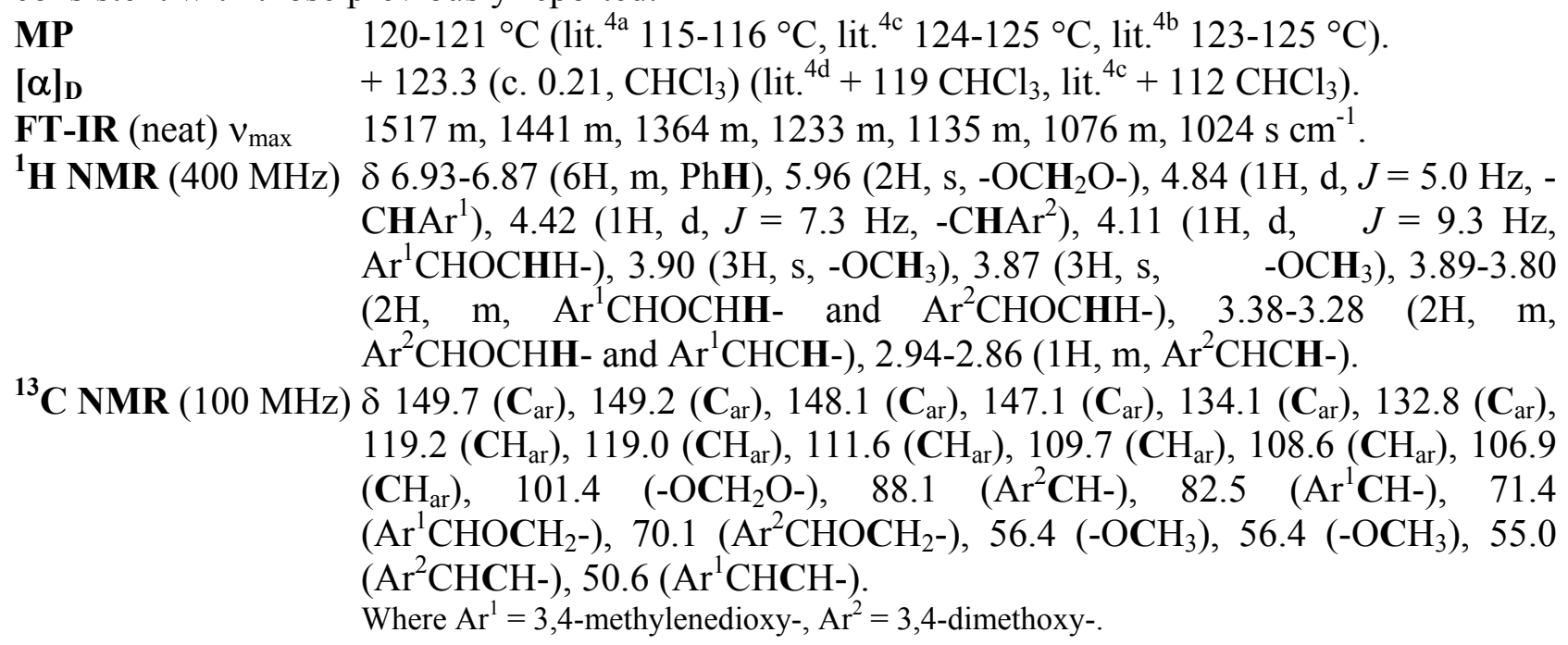

4 (a) Yoshida, S.; Ogiku, T.; Ohmizu, H.; Iwasaki, T. J. Org. Chem. 1997, 62, 1310. (b) Yoshida, S.; Ogiku, T.; Ohmizu, H.; Iwasaki, T. Tetrahedron Lett. 1995, 36, 1455-1458. (c) Bytheway, I. R.; Ghisalberti, E. L.; Gotsis, S.; Jefferies, P. R.; Skelton, B. W.; Sugars, K. E.; White, A. H. Aust. J. Chem. 1987, 40, 1913-1917. (d) Gunatilaka, A. A.; Silva, A. M. Y.; Jasmin, D.; Sotheeswaran, S.; Tillekeratne, L. M. V. Phytochemistry 1982, 21, 2719-2724. 
General Methods. ${ }^{1} \mathrm{H}-\mathrm{NMR}$ and ${ }^{13} \mathrm{C}-\mathrm{NMR}$ were recorded on a 300 or $400 \mathrm{MHz}$ spectrometer $(300$ or $400 \mathrm{MHz},{ }^{1} \mathrm{H}-\mathrm{NMR}$ respectively and 75 or $100 \mathrm{MHz},{ }^{13} \mathrm{C}-\mathrm{NMR}$ respectively) in deuteriochloroform $\left(\mathrm{CDCl}_{3}\right)$ with chloroform $\left(\delta 7.26 \mathrm{ppm}{ }^{1} \mathrm{H}, \delta 77.00 \mathrm{ppm}{ }^{13} \mathrm{C}\right)$ as the internal standard. Infrared (IR) spectra are reported in wavenumbers $\left(\mathrm{cm}^{-1}\right)$. Melting points were obtained in open capillary tubes and are uncorrected. All non-aqueous reactions were carried out under an inert atmosphere, in oven-dried glassware. The following solvents were distilled before use: THF (from $\mathrm{Na}$ /benzophenone) and $\mathrm{CH}_{2} \mathrm{Cl}_{2}$ (from $\mathrm{CaH}_{2}$ ) and where appropriate, other reagents and solvents were purified by standard techniques. ${ }^{5}$ TLC was performed on glass-backed plates coated with silica gel 60 with an $\mathrm{F}_{254}$ indicator; the chromatograms were visualised under UV light and/or by staining with phosphomolybdic acid $\left(20 \%\right.$ solution in ethanol) or $\mathrm{KMnO}_{4}$. Flash column chromatography was either performed with 40-63 $\mu \mathrm{m}$ silica gel (Merck) in standard glass columns or using pre-packed columns on a FlashMaster Personal Chromatography apparatus supplied by Jones Chromatography. Column dimensions are quoted in $\mathrm{cm}$ (width $\mathrm{x}$ height).

${ }^{5}$ Perin, D. D.; Armarego, W. L. F. Purification of laboratory chemicals; 3rd ed.; Butterworth-Heinemann Ltd.: Oxford, 1994. 